\title{
Maintaining Matroid 3-Connectivity With Respect to a Fixed Basis
}

\author{
Alan Williams
}

A thesis

submitted to Victoria University of Wellington in fulfilment of the requirements for the degree of Master of Science in Mathematics.

Victoria University of Wellington

2010 


\begin{abstract}
We show that for any 3-connected matroid $M$ on a ground set of at least four elements such that $M$ does not contain any 4-element fans, and any basis $B$ of $M$, there exists a set $K \subseteq E(M)$ of four distinct elements such that for all $k \in K, \operatorname{si}(M / k)$ is 3-connected whenever $k \in B$, and $\operatorname{co}(M \backslash k)$ is 3-connected whenever $k \in E(M)-B$. Moreover, we show that if no other elements of $E(M)-K$ satisfy this property, then $M$ necessarily has path-width 3 .
\end{abstract}




\section{Acknowledgements}

I would like to express my gratitude to Geoff Whittle, for without his wise guidance \& supervision, this thesis would not exist.

Thanks also to my parents, for I would not be here without them. 


\section{Contents}

1 Introduction 1

2 Matroids 4

2.1 Matroids; Independent Sets . . . . . . . . . . . . . . 4

2.2 Representable Matroids . . . . . . . . . . . . . . . . . 6

2.3 Rank . . . . . . . . . . . . . . . . . 8

2.4 Closure . . . . . . . . . . . . . . . . . . . 9

2.5 Duality . . . . . . . . . . . . . . . . 10

2.6 Minors . . . . . . . . . . . . . . . . . 13

2.7 Rota's Conjecture . . . . . . . . . . . . . . . 16

3 Connectivity 20

3.1 Introduction . . . . . . . . . . . . . . . . 20

3.2 Various Lemmas on 3-connectivity . . . . . . . . . . . . . 22

3.3 Vertical \& Cyclic Separations . . . . . . . . . . . . . . 25

3.4 Further Results on Connectivity . . . . . . . . . . . . . . . 29

3.5 Fixed Basis Connectivity . . . . . . . . . . . . . . . . 37

4 The Existence of Removable Elements 41

4.1 Preliminary Results . . . . . . . . . . . . . . . . . . . . . . . . 41

4.2 The Minimal Number of Removable

Elements . . . . . . . . . . . . . . . 46 
CONTENTS

5 Matroids with Minimal Removability 49

5.1 Preliminary Constructions . . . . . . . . . . . . . . . . . . . . 49

5.2 Arranging Removable Elements . . . . . . . . . . . . . . 60

5.3 Path Width . . . . . . . . . . . . . . . . . . . 69

6 Future Work $\quad 74$

6.1 Some Conjectures . . . . . . . . . . . . . . . . . . 74 


\section{Chapter 1}

\section{Introduction}

It is well established that the class of 3-connected matroids is fundamental in matroid theory. Indeed, any matroid that is not 3-connected can be constructed from a collection of its 3 -connected minors by a sequence of direct sums and 2-sums. Moreover, when working with representable matroids, 3connectivity is an essential ingredient in obtaining any sense of uniqueness of representations.

Bixby's Lemma [3] (see Lemma 3.4.2) is a useful tool for maintaining matroid 3-connectivity. It states that for any element $e$ of a 3-connected matroid $M$, either $M / e$ is 3-connected up to parallel classes, or $M \backslash e$ is 3-connected up to series classes. However, situations arise where we have some particular type of element of a 3-connected matroid, and we specifically want to contract it (or specifically want to delete it) whilst maintaining 3-connectivity. In such situations, Bixby's Lemma may be of little use.

For example, if we have a representation $\left[I_{r}, D\right]$ of a matroid $M$ in standard form, then the columns of $I_{r}$ correspond to a basis $B$ of $M$, while the columns of $D$ correspond to a cobasis $E(M)-B$ of $M$. We can now suppress mention of the identity submatrix $I_{r}$, and take $D$ to be our representation of $M$; where rows of $D$ correspond to the basis $B$ and columns of $D$ to the cobasis $E(M)-B$. Suppose then that we want to study representations of minors of $M$. If we restrict ourselves to those minors that can be obtained via contracting elements of the basis $B$ or deleting elements of the cobasis $E(M)-B$, then a representation for such a minor can be found simply by deleting rows or columns of the matrix $D$. Doing so leaves all remaining 
entries of $D$ unchanged, so that important structural information that may be present in $D$ can be preserved.

Oxley, Semple, and Whittle [10] studied this precise situation. One result of theirs was that for a 3-connected matroid $M$ with no 4-element fans, and any basis $B$, there always exists either some element $b \in B$ such that $M / b$ is 3 -connected, or some element $b^{*} \in E(M)-B$ such that $M \backslash b^{*}$ is 3-connected. In this study, we were interested in extending this result in order to obtain a set $K$ of as many elements as possible such that whenever $e \in K \cap B, M / e$ is 3 -connected up to parallel classes, and whenever $e \in K \cap(E(M)-B), M \backslash e$ is 3-connected up to series classes. In Theorem 4.0.1, we show that provided $|E(M)| \geq 4, M$ always contains at least four such elements. We then looked at situations where this lower bound is met; and concluded with the result that in such a case, the matroid $M$ must have path-width 3 (Theorem 5.0.2).

While the focus of this work was predominantly on the original research undertaken, I have attempted to make the thesis as self contained as possible. Definitions are given for all terminology and notation used, and proofs are provided for all results that are utilised. We use the notation $\subseteq$ to mean subset and $\subset$ for proper subset. Chapters 2 and 3 give a short introduction to matroid theory and matroid connectivity. Chapter 4 is concerned with finding a set consisting of elements which can be removed whilst maintaining 3 -connectivity up to parallel and series classes; and culminates with Theorem 4.0.1. In Chapter 5, we study those situations where exactly four such elements are found. Within this chapter, Sections 5.1 and 5.2 present results on how we may arrange certain key elements and substructures present in our matroids with respect to 3 -separations. Section 5.3 then ties these results in with the notion of path-width; concluding with Theorem 5.0.2.

As we neared a completed proof of Theorem 5.0.2, the fog of war that had surrounded the problem lifted greatly. It is now clear that there is a slightly more efficient route that can be used to prove the result. In particular, some constructions present in Section 5.1 can be largely circumvented. Nevertheless, we have opted to include and use them, as they do give some insight into the possible structures that can arise. We defer the use of the simplified argument to a research paper.

With regards to the originality of the material presented herein, Chapter 2 consists exclusively of background material on matroid theory, and as such, is all well known. Likewise for Chapter 3; with the exception of 3.4.4-3.4.8; 
which are all original, and 3.5.1; where a new definition is made. Everything in Chapter 4 is original work. Theorem 5.0.2 is new, as is the material contained in Sections 5.1 and 5.2. Definition 5.3.1 is given in [5], and the results of Lemmas 5.3.2 -5.3.4 are known. The remainder of Section 5.3 is original work. 


\section{Chapter 2}

\section{Matroids}

The purpose of this chapter is to define matroids and take a look at some of their basic properties. The introduction is brief, and material presented herein has been chosen according to what background knowledge is explicitly required for an understanding of the thesis as a whole. For a thorough introduction to matroid theory, see Matroid Theory by Oxley [9].

\subsection{Matroids; Independent Sets}

Matroids can be defined by means of a number of equivalent axiom schemes. The definition we give here is, arguably, perhaps the most intuitive.

Definition 2.1.1. A matroid is a pair $(E, \mathcal{I})$ where $E$ is a finite set, and $\mathcal{I} \subseteq \mathcal{P}(E)$ satisfying the following axioms:

(I1) $\emptyset \in \mathcal{I}$.

(I2) If $I_{1} \in \mathcal{I}$ and $I_{2} \subseteq I_{1}$, then $I_{2} \in \mathcal{I}$.

(I3) If $I_{1}, I_{2} \in \mathcal{I}$ and $\left|I_{1}\right|<\left|I_{2}\right|$, then there exists some $e \in I_{2}-I_{1}$ such that $I_{1} \cup\{e\} \in \mathcal{I}$.

If $(E, \mathcal{I})$ is a matroid, we say that $(E, \mathcal{I})$ is a matroid on $E$. $E$ is the ground set of the matroid. The members of $\mathcal{I}$ are the independent sets of the matroid. 
Members of $\mathcal{P}(E)-\mathcal{I}$ are called dependent sets. We usually simply denote matroids by a capital letter such as $M$, and whenever we wish to explicitly refer to either the ground set of $M$ or the collection of independent sets of $M$, we use the notation $E(M)$ or $\mathcal{I}(M)$ respectively.

Definition 2.1.2. A basis of a matroid $M$ is a maximal independent set of $M$.

Lemma 2.1.3. [9, Lemma 1.2.1] If $B_{1}$ and $B_{2}$ are bases of a matroid $M$, then $\left|B_{1}\right|=\left|B_{2}\right|$.

Proof. Suppose $B_{1}, B_{2}$ are bases of some matroid $M$ where $\left|B_{1}\right|<\left|B_{2}\right|$. Then by (I3), there exists some $e \in B_{2}-B_{1}$ such that $B_{1} \cup\{e\}$ is independent. This contradicts the maximality of $B_{1}$. Hence $\left|B_{1}\right| \geq\left|B_{2}\right|$, and similarly, $\left|B_{2}\right| \geq\left|B_{1}\right|$.

Lemma 2.1.4. [9, Lemma 1.2.2] Let $B_{1}$ and $B_{2}$ be bases of a matroid $M$ and $x \in B_{1}-B_{2}$. Then there exists some $y \in B_{2}-B_{1}$ such that $\left(B_{1}-\{x\}\right) \cup\{y\}$ is a basis of $M$.

Proof. Both $B_{1}-\{x\}$ and $B_{2}$ are independent, and by Lemma 2.1.3, $\mid B_{1}-$ $\{x\}|<| B_{2} \mid$. Therefore, by (I3), there exists some $y \in B_{2}-\left(B_{1}-\{x\}\right)$ such that $\left(B_{1}-\{x\}\right) \cup\{y\}$ is independent. Now $\left(B_{1}-\{x\}\right) \cup\{y\}$ is contained in some basis of $M$, and $\left|\left(B_{1}-\{x\}\right) \cup\{y\}\right|=\left|B_{1}\right|$, so that by Lemma 2.1.3, $\left(B_{1}-\{x\}\right) \cup\{y\}$ is itself a basis of $M$.

Definition 2.1.5. A circuit of a matroid $M$ is a minimal dependent set. Circuits which consist of a single element are called loops, two element circuits are referred to as a parallel pair, and a circuit of size three is a triangle.

Lemma 2.1.6. [9, Lemma 1.1.3] Let $C_{1}$ and $C_{2}$ be distinct circuits of a matroid $M$ and $e \in C_{1} \cap C_{2}$. Then there exists a circuit $C_{3}$ of $M$ such that $C_{3} \subseteq\left(C_{1} \cup C_{2}\right)-\{e\}$.

Proof. Suppose $\left(C_{1} \cup C_{2}\right)-\{e\}$ is independent. Taking any $x \in C_{2}-C_{1} \neq \emptyset$, we have that $C_{2}-\{x\}$ is independent. Now let $I$ be a maximal independent set of $C_{1} \cup C_{2}$ such that $I$ contains $C_{2}-\{x\}$. Then $x \notin I$ and there exists some $y \in C_{1}-I$. Therefore

$$
|I| \leq\left|\left(C_{1} \cup C_{2}\right)-\{x, y\}\right|=\left|C_{1} \cup C_{2}\right|-2<\left|\left(C_{1} \cup C_{2}\right)-\{e\}\right| .
$$


Now applying (I3), we conclude that there must exist an independent $I^{\prime}$ such that $C_{2}-\{x\} \subseteq I^{\prime} \subseteq C_{1} \cup C_{2}$ and $\left|I^{\prime}\right|>|I|$. The contradicts the maximality of $I$.

Lemma 2.1.7. Let $B_{1}$ and $B_{2}$ be bases of a matroid $M$ and $x \in B_{2}-B_{1}$. Then there exists some $y \in B_{1}-B_{2}$ such that $\left(B_{1}-\{y\}\right) \cup\{x\}$ is a basis of $M$.

Proof. As $B_{1} \cup\{x\}$ is dependent, it must contain a circuit $C_{1}$. Take any $y \in C_{1}-B_{2} \neq \emptyset$ and consider $\left(B_{1}-\{y\}\right) \cup\{x\}$. If $\left(B_{1}-\{y\}\right) \cup\{x\}$ contains a circuit $C_{2}$, then by Lemma 2.1.6, there exists a circuit $C_{3}$ such that $C_{3} \subseteq$ $\left(C_{1} \cup C_{2}\right)-\{x\} \subseteq B_{1}$, but this contradicts the fact that $B_{1}$ is independent. Therefore $\left(B_{1}-\{y\}\right) \cup\{x\}$ must be independent. As $\left|\left(B_{1}-\{y\}\right) \cup\{x\}\right|=\left|B_{1}\right|$, it then follows from Lemma 2.1.3 that $\left(B_{1}-\{y\}\right) \cup\{x\}$ is a basis of $M$.

\section{$2.2 \quad$ Representable Matroids}

Matroids were originally introduced by Whitney in 1935 [16]; largely in an attempt to capture the notion of linear independence present in vector spaces in an axiomatic manner.

Proposition 2.2.1. Let $\mathbb{F}$ be a field and $n \in \mathbb{Z}^{+}$. Let $E$ be a multiset of vectors from $\mathbb{F}^{n}$. Define

$$
\mathcal{I}=\{I \subseteq E: I \text { linearly independent }\}
$$

Then $(E, \mathcal{I})$ is a matroid.

Proof. $\mathcal{I}$ clearly satisfies (I1) and (I2). Suppose $I_{1}$ and $I_{2}$ are linearly independent subsets of $E$ where $\left|I_{1}\right|<\left|I_{2}\right|$. Let $W=\operatorname{Span}\left(I_{1} \cup I_{2}\right) \subseteq \mathbb{F}^{n}$. Letting $\operatorname{dim}(W)$ denote the dimension of $W$, we have $\left|I_{2}\right| \leq \operatorname{dim}(W)$. Assume that for all $e \in I_{2}-I_{1}, I_{1} \cup\{e\}$ is linearly dependent. Then $W \subseteq \operatorname{Span}\left(I_{1}\right)$ and

$$
\left|I_{2}\right| \leq \operatorname{dim}(W) \leq\left|I_{1}\right|<\left|I_{2}\right|
$$

which is absurd. Hence there must exist some $e \in I_{2}-I_{1}$ such that $I_{1} \cup\{e\}$ is linearly independent; this gives (I3). 
Suppose that $A$ is some $n \times p$ matrix over a field $\mathbb{F}$. Then the collection of columns of $A$ correspond to a multiset of vectors from $\mathbb{F}^{n}$. In light of Proposition 2.2.1 above, there is a matroid which has the collection of columns of $A$ as its ground set; in which the independent sets are precisely the sets of columns of $A$ which are linearly independent over $\mathbb{F}^{n}$. We write $M[A]$ to represent the matroid obtained from a matrix $A$ in this manner. Such a matroid is said to be representable. In particular, if $M=M[A]$ is some matroid obtained from a matrix $A$ over $\mathbb{F}$, then $M$ is said to be representable over $\mathbb{F}$.

We see that Whitney [16] did indeed capture the notion of linear independence to some extent. But do matroids correspond identically to those structures which arise from configurations of vectors as in the above proposition? The following example due to Vámos [15] answers this question in the negative; the class of representable matroids is strictly contained within the class of matroids.

Example 2.2.2. Let $E=\{1,2,3,4,5,6,7,8\}$ and

$$
\begin{aligned}
\mathcal{I}= & \{X: X \subset E,|X| \leq 4\}- \\
& \{\{1,2,3,4\},\{1,4,5,6\},\{1,4,7,8\},\{2,3,5,6\},\{2,3,7,8\}\}
\end{aligned}
$$

Then $(E, \mathcal{I})$ is a matroid: the Vámos matroid, which is not representable over any field.

So can we somehow classify those matroids which are representable? Perhaps it would be possible to tighten our definition of a matroid as to exclude non-representable structures? As the following example illustrates, we need to specify which particular field $\mathbb{F}$ we are considering when determining whether or not a matroid $M$ is representable.

Example 2.2.3. Let $E=\{a, b, c, d\}$ and $\mathcal{I}=\{X: X \subset E,|X| \leq 2\}$. Then $M=(E, \mathcal{I})$ is readily seen to be a matroid which is representable over $\mathbb{R}$. However, a simple combinatorial examination reveals that $M$ is not representable over $G F(2)$. This particular matroid is denoted $U_{2,4}$.

So fix some field $\mathbb{F}$. Now given an arbitrary matroid $M$, how do we decide whether or not $M$ is $\mathbb{F}$ representable? This question is a natural one, and one that has been studied extensively. Yet it remains largely unanswered. In Section 2.7, we shall survey one approach that has been taken in an attempt to solve this problem. 


\subsection{Rank}

Definition 2.3.1. Let $M=(E, \mathcal{I})$ be a matroid. Then for any $X \subseteq E$, the rank of $X$; denoted $r(X)$, is the size of the largest independent set contained in $X$. We write $r(M)$ to represent $r(E(M))$ : the rank of $M$. When we are dealing with multiple matroids, we often write $r_{M}(X)$ to denote the rank of the set $X$ in the matroid $M$.

It follows from Lemma 2.1.3 that the rank of a matroid is equal to the size of any basis for that matroid.

Lemma 2.3.2. Let $M=(E, \mathcal{I})$ be a matroid. For any $X, Y \subseteq E$,

$$
r(X \cup Y)+r(X \cap Y) \leq r(X)+r(Y)
$$

Proof. If $r(X \cup Y)=r(X \cap Y)$, this is immediate. So assume that is not the case. Let $A$ be a maximally sized independent set contained in $X \cap Y$. Let $A^{\prime}$ be a maximally sized independent set contained in $X \cup Y$. Then $|A|<\left|A^{\prime}\right|$, and so, by repeatedly applying (I3), we can extend $A$ to a maximally sized independent set of $X \cup Y$. Letting $B$ denote this extension of $A$, we have $r(X \cup Y)=|B|$ and $r(X \cap Y)=|A|$. Now we have

$$
\begin{aligned}
r(X)+r(Y) & \geq|B \cap X|+|B \cap Y| \\
& =|B \cap(X \cup Y)|+|B \cap X \cap Y| \\
& =|B|+|A| \\
& =r(X \cup Y)+r(X \cap Y) .
\end{aligned}
$$

If $M$ is a matroid and $X \subseteq E(M)$ is such that $r(X)=2$, then we call $X$ a line of $M$. When $L$ is a line of $M$ with $|L|=n$, we often say that $L$ is a $n$-point line. Note that some authors take lines of matroids to be rank 2 flats. In this thesis, lines are simply rank 2 sets, and need not be flats. We present two simple results about lines of matroids:

Lemma 2.3.3. Let $M$ be a matroid containing a n-point line $L$, and let $B$ be a basis of $M$. Then $|L \cap B| \leq 2$. 
Proof. If $|L \cap B| \geq 3$, then $r(L \cap B) \geq 3$ because $B$ is independent. But $r(L \cap B) \leq r(L)=2$, so $|L \cap B| \leq 2$.

Lemma 2.3.4. Let $M$ be a matroid with 4-point lines $L_{1}, L_{2} \subseteq E(M)$. If $r\left(L_{1} \cap L_{2}\right) \geq 2$, then either:

(i) $L_{1}=L_{2}$, or

(ii) $L_{1} \cup L_{2}$ is a line with $\left|L_{1} \cup L_{2}\right| \geq 5$.

Proof. Suppose $r\left(L_{1} \cap L_{2}\right) \geq 2$ and $L_{1} \neq L_{2}$. Then certainly, $\left|L_{1} \cup L_{2}\right| \geq 5$, and, by Lemma 2.3.2, $r\left(L_{1} \cup L_{2}\right) \leq r\left(L_{1}\right)+r\left(L_{2}\right)-r\left(L_{1} \cap L_{2}\right)=2$.

If $M=M[A]$ is some representable matroid, it follows from elementary linear algebra that the rank of $M$ corresponds identically to the dimension of the rowspace of the matrix $A$ : the number of non-zero rows in a reduced row echelon form of $A$.

\subsection{Closure}

Definition 2.4.1. Let $M$ be a matroid. Then for all $X \subseteq E(M)$, the closure of $X$; denoted $\operatorname{cl}(X)$ is defined by

$$
\operatorname{cl}(X)=\{x \in E(M): r(X \cup\{x\})=r(X)\} .
$$

A set $X \subseteq E(M)$ is closed if $\operatorname{cl}(X)=X$. If $X \subseteq E(M)$ is a closed set with $r(X)=r(M)-1$, then we refer to $X$ as a hyperplane of $M$. We say that $Y \subseteq E(M)$ is spanning if $\operatorname{cl}(Y)=E(M)$. When dealing with multiple matroids, we often write $\operatorname{cl}_{M}(X)$ to denote the closure of $X$ in the matroid $M$.

The following result is used freely throughout this thesis.

Lemma 2.4.2. Let $M$ be a matroid and $X \subseteq E(M)$. Then $X$ is a circuit if and only if $X$ is a minimal set with the property that for all $x \in X$, $x \in \operatorname{cl}(X-\{x\})$.

Proof. $X$ is a circuit iff $X$ is a minimal dependent set; iff $X$ is dependent and for all $x \in X, X-\{x\}$ is independent; iff $X$ is a minimal set such that for all $x \in X, r(X-\{x\})=r(X)$; iff $X$ is a minimal set with the property that for all $x \in X, x \in \operatorname{cl}(X-\{x\})$. 


\subsection{Duality}

For any matroid $M$ with ground set $E$, there is a unique dual matroid $M^{*}$ on $E$ defined as follows.

Proposition 2.5.1. Let $M=(E, \mathcal{I})$ be a matroid, and let $\mathcal{B}$ denote the collection of bases of $M$. Let

$$
\begin{gathered}
\mathcal{B}^{*}=\{E-B: B \in \mathcal{B}\}, \text { and } \\
\mathcal{I}^{*}=\left\{I \subseteq E: I \subseteq B^{*} \text { for some } B^{*} \in \mathcal{B}^{*}\right\}
\end{gathered}
$$

Then $M^{*}=\left(E, \mathcal{I}^{*}\right)$ is a matroid.

Proof. As $\mathcal{I} \neq \emptyset, \mathcal{B} \neq \emptyset$, so $\mathcal{B}^{*} \neq \emptyset$ and $\mathcal{I}^{*} \neq \emptyset$; hence (I1) is satisfied. Suppose $I_{1} \in \mathcal{I}^{*}$. Then $I_{1} \subseteq B_{1}^{*}$ for some $B_{1}^{*} \in \mathcal{B}^{*}$. Therefore for any $I_{2} \subseteq I_{1}, I_{2} \subseteq B_{1}^{*}$ also, so that $I_{2} \in \mathcal{I}^{*}$; which confirms that (I2) holds. To show that $M^{*}=\left(E, \mathcal{I}^{*}\right)$ satisfies (I3), we first note the following:

2.5.1.1. If $B_{1}^{*}, B_{2}^{*} \in \mathcal{B}^{*}$ and $x \in B_{1}^{*}-B_{2}^{*}$, then there exists some $y \in B_{2}^{*}-B_{1}^{*}$ such that $\left(B_{1}^{*}-\{x\}\right) \cup\{y\} \in \mathcal{B}^{*}$.

Proof. Suppose $B_{1}^{*}, B_{2}^{*} \in \mathcal{B}^{*}$ and $x \in B_{1}^{*}-B_{2}^{*}$. Then there exist bases $B_{1}=B_{1}^{*}-E$ and $B_{2}=B_{2}^{*}-E$ of $M$ such that $x \in B_{2}-B_{1}$. Now by Lemma 2.1.7, there exists some $y \in B_{1}-B_{2}$ such that $\left(B_{1}-\{y\}\right) \cup\{x\}$ is a basis of $M$. Therefore $E-\left(\left(B_{1}-\{y\}\right) \cup\{x\}\right)=\left(B_{1}^{*}-\{x\}\right) \cup\{y\} \in \mathcal{B}^{*}$.

Assume that there exists $I_{1}, I_{2} \in \mathcal{I}^{*}$, where $\left|I_{1}\right|<\left|I_{2}\right|$ and for all $e \in$ $I_{2}-I_{1}, I_{1} \cup\{e\} \notin \mathcal{I}^{*}$. By definition, there exists $B_{1}^{*}, B_{2}^{*} \in \mathcal{B}^{*}$ such that $I_{1} \subseteq B_{1}^{*}$ and $I_{2} \subseteq B_{2}^{*}$. Let $B_{1}^{*}$ be any such element of $\mathcal{B}^{*}$, and choose $B_{2}^{*} \in \mathcal{B}^{*}$ such that $\left|B_{2}^{*}-\left(I_{2} \cup B_{1}^{*}\right)\right|$ is minimal.

Suppose there exists some $x \in B_{2}^{*}-\left(I_{2} \cup B_{1}^{*}\right)$. Then by 2.5.1.1, there exists some $y \in B_{1}^{*}-B_{2}^{*}$ such that $\left(B_{2}^{*}-\{x\}\right) \cup\{y\} \in \mathcal{B}^{*}$. But then $\left|\left(\left(B_{2}^{*}-\{x\}\right) \cup\{y\}\right)-\left(I_{2} \cup B_{1}^{*}\right)\right|<\left|B_{2}^{*}-\left(I_{2} \cup B_{1}^{*}\right)\right|$; which contradicts our choice of $B_{2}^{*}$. Therefore $B_{2}^{*}-\left(I_{2} \cup B_{1}^{*}\right)=\emptyset$, and it follows that $B_{2}^{*}-B_{1}^{*}=I_{2}-B_{1}^{*}$, which implies that $B_{2}^{*}-B_{1}^{*}=I_{2}-I_{1}$.

Now suppose there exists some $x \in B_{1}^{*}-\left(I_{1} \cup B_{2}^{*}\right)$. Then by 2.5.1.1, there exists some $y \in B_{2}^{*}-B_{1}^{*}$ such that $\left(B_{1}^{*}-\{x\}\right) \cup\{y\} \in \mathcal{B}^{*}$. Now $I_{1} \cup\{y\} \subseteq$ 
$\left(B_{1}^{*}-\{x\}\right) \cup\{y\} \in \mathcal{B}^{*}$. Hence $I_{1} \cup\{y\} \in \mathcal{I}^{*}$. But $B_{2}^{*}-B_{1}^{*}=I_{2}-I_{1}$, so $y \in I_{2}$, but this contradicts our assumption that for all $e \in I_{2}-I_{1}, I_{1} \cup\{e\} \notin \mathcal{I}^{*}$. Therefore $B_{1}^{*}-\left(I_{1} \cup B_{2}^{*}\right)=\emptyset$, which implies that $B_{1}^{*}-B_{2}^{*}=I_{1}-B_{2}^{*}$, and so $B_{1}^{*}-B_{2}^{*} \subseteq I_{1}-I_{2}$.

By Lemma 2.1.3, $\left|B_{1}^{*}\right|=\left|B_{2}^{*}\right|$, so $\left|B_{1}^{*}-B_{2}^{*}\right|=\left|B_{2}^{*}-B_{1}^{*}\right|$. But we've seen that $B_{2}^{*}-B_{1}^{*}=I_{2}-I_{1}$ and $B_{1}^{*}-B_{2}^{*} \subseteq I_{1}-I_{2}$, therefore $\left|I_{2}-I_{1}\right| \leq\left|I_{1}-I_{2}\right|$; which contradicts the fact that $\left|I_{2}\right|>\left|I_{1}\right|$. We are left to conclude that there indeed must exist some $e \in I_{2}-I_{1}$ such that $I_{1} \cup\{e\} \in \mathcal{I}^{*}$; which proves (I3). Therefore $M^{*}=\left(E, \mathcal{I}^{*}\right)$ is a matroid.

Let $M^{*}$ be a matroid obtained from a matroid $M$ as in the above proposition. Then $M^{*}$ is said to be the dual of $M$. A basis of $M^{*}$ is called a cobasis of $M$. Similarly, independent sets, circuits, hyperplanes, spanning sets and lines of $M^{*}$ are referred to as coindependent sets, cocircuits, cohyperplanes, cospanning sets, and colines of $M$ respectively. Whenever $\Gamma$ is a coline of a matroid with $|\Gamma|=n$, we say that $\Gamma$ is a $n$-point coline. One, two, and three element cocircuits of $M$ are called coloops, series pairs and triads respectively. Note also that $\left(M^{*}\right)^{*}=M$.

Lemma 2.5.2. Let $M$ be a matroid on a ground set $E$, and $X \subseteq E$. Then

(i) $X$ is coindependent if and only if $E-X$ is spanning, and

(ii) $X$ is a cohyperplane if and only if $E-X$ is a circuit.

Proof. $X$ is coindependent iff $X$ is contained in a cobasis; iff $E-X$ contains a basis; iff $r(E-X)=r(M)$; iff $\operatorname{cl}(E-X)=E$; iff $E-X$ is spanning. It then follows that $X$ is a cohyperplane iff $X$ is a maximal non-cospanning set; iff $E-X$ is a minimal dependent set; iff $E-X$ is a circuit.

Let $M$ be a matroid. For any $X \subseteq E(M)$, the corank of $X$; denoted $r^{*}(X)$ is equal to $r_{M^{*}}(X)$. The coclosure of $X$; denoted $\mathrm{cl}^{*}(X)$ is equal to $\mathrm{cl}_{M^{*}}(X)$. A set $X$ is coclosed if $\mathrm{cl}^{*}(X)=X$.

The corank function of a matroid satisfies the following: 
Lemma 2.5.3. Let $M$ be a matroid on a ground set $E$. For any $X \subseteq E$, $r^{*}(X)=|X|-r(M)+r(E-X)$.

Proof. Take any $X \subseteq E$. Let $I^{*}$ be a maximally sized coindependent set contained in $X$. By Lemma 2.5.2, $E-I^{*}$ is spanning. Hence $E-I^{*}$ must contain a basis $B$. It then follows that $X \cap B=X-I^{*}$.

If $r(E-X) \geq|B-X|$, then by (I3), there exists an element $e \in E-$ $(X \cup B)$ such that $(B-X) \cup\{e\}$ is independent. Therefore $(B-X) \cup\{e\}$ is contained in some basis $B^{\prime}$. But then $X-B^{\prime}$ is coindependent by Lemma 2.5.2 and $\left|X-B^{\prime}\right|>|X-B|=I^{*}$; which contradicts our choice of $I^{*}$. So we must have $r(E-X)=|B-X|$, and so:

$$
\begin{aligned}
|X| & =\left|X \cap I^{*}\right|+|X \cap B| \\
& =r^{*}(X)+|X \cap B| \\
& =r^{*}(X)+|B|-|B-X| \\
& =r^{*}(X)+r(M)-|B-X| \\
& =r^{*}(X)+r(M)-r(E-X) .
\end{aligned}
$$

Lemma 2.5.4. Let e be an element of a matroid $M$, and let $X$ and $Y$ be disjoint sets whose union is $E(M)-\{e\}$. Then $e \in \operatorname{cl}(X)$ if and only if $e \notin \mathrm{cl}^{*}(Y)$.

Proof. By Lemma 2.5.3, $r^{*}(Y)=|Y|-r(M)+r(X \cup\{e\})$ and $r^{*}(Y \cup\{e\})=$ $|Y|+1-r(M)+r(X)$. Therefore $r^{*}(Y \cup\{e\})=r^{*}(Y)+1+(r(X)-r(X \cup\{e\}))$, so that $e \in \operatorname{cl}(X)$ iff $r^{*}(Y) \neq r^{*}(Y \cup\{e\})$; iff $e \notin \operatorname{cl}^{*}(Y)$.

Lemma 2.5.5. Let $M$ be a matroid containing a circuit $C$ and a cocircuit $C^{*}$. Then $\left|C \cap C^{*}\right| \neq 1$.

Proof. Suppose that $C \cap C^{*}=\{x\}$. Letting $H=E(M)-C^{*}$, it follows from Lemma 2.5.2 that $H$ is a hyperplane. Now $C-\{x\} \subseteq H$, and so, by Lemma 2.4.2, $x \in \operatorname{cl}(C-\{x\}) \subseteq \operatorname{cl}(H)$. But then $x \in \operatorname{cl}(H)-H$; which contradicts the fact that hyperplanes are closed. Therefore $\left|C \cap C^{*}\right| \neq 1$. 
Duality is a powerful tool in matroid theory. Whenever we prove that some result must hold for a matroid $M$, we may dualise the statement of that result to immediately obtain another result that holds for the matroid $M^{*}$. The following two facts about colines; which will be useful in what follows, are a good illustration of this.

Lemma 2.5.6. Let $M$ be a matroid containing an n-point coline $\Gamma$, and let $B$ be a basis of $M$. Then $|\Gamma \cap B| \geq n-2$.

Proof. By dualising Lemma 2.3.3, we have the following result: If $M$ is a matroid containing an $n$-point coline $L$, and $B$ is a cobasis of $M$, then $\mid L \cap$ $B \mid \leq 2$. Equivalently: if $M$ is a matroid containing an $n$-point coline $\Gamma$, and $E(M)-B$ is a basis of $M$, then $|L \cap(E(M)-B)| \geq n-2$. The result then follows via a relabelling of the basis $E(M)-B$.

Lemma 2.5.7. Let $M$ be a matroid with 4-point colines $\Gamma_{1}, \Gamma_{2} \subseteq E(M)$. If $r^{*}\left(\Gamma_{1} \cap \Gamma_{2}\right) \geq 2$, then either:

(i) $\Gamma_{1}=\Gamma_{2}$, or

(ii) $\Gamma_{1} \cup \Gamma_{2}$ is a coline with $\left|\Gamma_{1} \cup \Gamma_{2}\right| \geq 5$.

Proof. This follows immediately by dualising Lemma 2.3.4, and relabelling so that $L_{1}$ and $L_{2}$ become $\Gamma_{1}$ and $\Gamma_{2}$ respectively.

We now note that if $A$ is a representation of a matroid over some field $\mathbb{F}$, then the underlying matroid is invariant under applying standard row operations to the matrix $A$, as well as being invariant upon interchanging columns within $A$, and multiplying a column of $A$ by an element of $\mathbb{F}$. From this it is readily deduced that for a matroid $M$ representable over $\mathbb{F}$, the dual matroid $M^{*}$ is also representable over $\mathbb{F}$, and indeed, we can explicitly construct a representation of $M^{*}$. We say that the class of $\mathbb{F}$-representable matroids is closed under duality.

\subsection{Minors}

We now briefly examine the fundamental substructures of a matroid: its minors. One natural substructure of a matroid $M$ is found when we simply 
shrink the ground set $E(M)$ and observe the restriction of $M$ on this smaller ground set. More formally:

Definition 2.6.1. Let $M=(E, \mathcal{I})$ be a matroid and $e \in E$. Let

$$
\mathcal{I}(M \backslash e)=\{I \subseteq E-\{e\}: I \in \mathcal{I}\}
$$

Then $(E, \mathcal{I}(M \backslash e))$ is readily seen to be a matroid; called $M$ delete e, and denoted $M \backslash e$. We say that $M \backslash e$ is obtained from $M$ by deleting $e$.

Armed with duality, we explore another natural matroid substructure.

Definition 2.6.2. Let $M$ be a matroid and $e \in E(M)$. The matroid $M$ contract $e$; denoted $M / e$ is defined to be $M / e=\left(M^{*} \backslash e\right)^{*} . M / e$ is said to be obtained from $M$ by contracting $e$.

Lemma 2.6.3. Suppose that $M$ is a matroid on a ground set $E, e \in E$ and $X \subseteq E-\{e\}$. Then $r_{M / e}(X)=r_{M}(X \cup\{e\})-r_{M}(\{e\})$.

Proof. $r_{M / e}(X)=r_{\left(M^{*} \backslash e\right)^{*}}(X)$, so, by Lemma 2.5.3,

$$
\begin{aligned}
r_{M / e}(X) & =|X|-r\left(M^{*} \backslash e\right)+r_{M^{*} \backslash e}(E-(X \cup\{e\})) \\
& =|X|-r^{*}(E-\{e\})+r^{*}(E-(X \cup\{e\})) \\
& =|X|-|E-\{e\}|-r_{M}(\{e\})+|E-(X \cup\{e\})|+r_{M}(X \cup\{e\}) \\
& =|X|+1-|X \cup\{e\}|+r_{M}(X \cup\{e\})-r_{M}(\{e\}) \\
& =r_{M}(X \cup\{e\})-r_{M}(\{e\}) .
\end{aligned}
$$

Lemma 2.6.4. Let $M$ be a matroid with distinct $e_{1}, e_{2} \in E(M)$. Then

(i) $\left(M \backslash e_{1}\right) \backslash e_{2}=\left(M \backslash e_{2}\right) \backslash e_{1}$, and

(ii) $\left(M / e_{1}\right) / e_{2}=\left(M / e_{2}\right) / e_{1}$, and

(iii) $\left(M \backslash e_{1}\right) / e_{2}=\left(M / e_{2}\right) \backslash e_{1}$. 
Proof. A set $I \subseteq E(M)$ is independent in $\left(M \backslash e_{1}\right) \backslash e_{2}$ iff $I \subseteq E(M)-\left\{e_{1}, e_{2}\right\}$ and $I$ is independent in $M$; iff $I$ is independent in $\left(M \backslash e_{2}\right) \backslash e_{1}$; which proves (i). Now by the definition of contraction together with (i), we have

$$
\begin{aligned}
\left(M / e_{1}\right) / e_{2} & =\left(M^{*} \backslash e_{1}\right)^{*} / e_{2}=\left(\left(M^{*} \backslash e_{1}\right) \backslash e_{2}\right)^{*}=\left(\left(M^{*} \backslash e_{2}\right) \backslash e_{1}\right)^{*} \\
& =\left(\left(M^{*} \backslash e_{2}\right)^{*} / e_{1}=\left(\left(M^{*}\right)^{*} / e_{2}\right) / e_{1}=\left(M / e_{2}\right) / e_{1}\right.
\end{aligned}
$$

so that (ii) holds. To prove (iii), take any $X \subseteq E(M)-\left\{e_{1}, e_{2}\right\}$. Then by Lemma 2.6.3,

$$
\begin{aligned}
r_{\left(M / e_{2}\right) \backslash e_{1}}(X) & =r_{M / e_{2}}(X) \\
& =r_{M}\left(X \cup\left\{e_{2}\right\}\right)-r_{M}\left(\left\{e_{2}\right\}\right) \\
& =r_{M \backslash e_{1}}\left(X \cup\left\{e_{2}\right\}\right)-r_{M \backslash e_{1}}\left(\left\{e_{2}\right\}\right) \\
& =r_{\left(M \backslash e_{1}\right) / e_{2}}(X) .
\end{aligned}
$$

Therefore the rank in $\left(M \backslash e_{1}\right) / e_{2}$ of any subset $X \in E(M)-\left\{e_{1}, e_{2}\right\}$ is equal to the rank of the same subset in the matroid $\left(M / e_{2}\right) \backslash e_{1}$. As $E(M)-\left\{e_{1}, e_{2}\right\}$ is the ground set of both of these matroids, it follows that the collection of independent sets of $\left(M \backslash e_{1}\right) / e_{2}$ is identical to the collection of independent sets of $\left(M / e_{2}\right) \backslash e_{1}$; we conclude that $\left(M \backslash e_{1}\right) / e_{2}=\left(M / e_{2}\right) \backslash e_{1}$.

As a consequence of the above result we see that the operations of deletion and contraction commute with both themselves and one another. Therefore any sequence of deletions and contractions from a matroid $M$ can be written in the form $M \backslash X / Y$ where $X$ and $Y$ are disjoint subsets of the ground set. Matroids obtained from a given matroid $M$ through any such sequence of deletions and contractions are called minors of $M$. If $M$ is a matroid with a minor $N$ where $N \neq M$, then we say that $N$ is a proper minor of $M$. As we shall see in Section 2.7, knowledge of the minors contained in some matroid $M$ can give us important information about some of the necessary properties of $M$.

The following is an extension of Lemma 2.6.3, which will be useful later on.

Lemma 2.6.5. Let $M$ be a matroid and $T \subseteq E(M)$. Then for all $X \subseteq$ $E(M)-T, r_{M / T}(X)=r_{M}(X \cup T)-r_{M}(T)$. 
Proof. Suppose $X \subseteq E(M)-T$ and let $T=\left\{e_{1}, \ldots, e_{n}\right\}$. By Lemma 2.6.3, $r_{M / e_{1}}(X)=r_{M}\left(X \cup\left\{e_{1}\right\}\right)-r_{M}\left(\left\{e_{1}\right\}\right)$, and it follows that

$$
\begin{aligned}
r_{M /\left\{e_{1}, e_{2}\right\}}(X) & =r_{M / e_{1}}\left(X \cup\left\{e_{2}\right\}\right)-r_{M / e_{1}}\left(\left\{e_{2}\right\}\right) \\
& =r_{M}\left(X \cup\left\{e_{1}, e_{2}\right\}\right)-r_{M}\left(\left\{e_{1}\right\}\right)-r_{M}\left(\left\{e_{1}, e_{2}\right\}\right)+r_{M}\left(\left\{e_{1}\right\}\right) \\
& =r_{M}\left(X \cup\left\{e_{1}, e_{2}\right\}\right)-r_{M}\left(\left\{e_{1}, e_{2}\right\}\right) .
\end{aligned}
$$

Now by an iteration of this argument for $e_{i} \in\left\{e_{3}, \ldots, e_{n}\right\}$, we can conclude that $r_{M /\left\{e_{1}, \ldots, e_{n}\right\}}(X)=r_{M}\left(X \cup\left\{e_{1}, \ldots, e_{n}\right\}\right)-r_{M}\left(\left\{e_{1}, \ldots, e_{n}\right\}\right)$ as required.

We conclude this section with a brief discussion on minors of representable matroids. If $M$ is a matroid which is representable over $\mathbb{F}$, and $e \in E(M)$, then we may obtain a representation for $M \backslash e$ simply by taking a representation of $M$ and deleting the column labelled by $e$. Also, we noted at the end of Section 2.5 that the class of $\mathbb{F}$-representable matroids is closed under duality. This implies that for $e \in E(M), M / e$ is also representable over $\mathbb{F}$. Let $A$ be a representation of $M$ over $\mathbb{F}$. If $e$ is a loop of $M$, then $M / e=M \backslash e$, so a representation of $M / e$ is found as above. So assuming that $e$ is not a loop of $M$, it turns out that to obtain a representation for $M / e$, we pivot on some non-zero entry of the column of $A$ labelled by $e$ to transform this column into a unit vector. Then, by deleting the row containing the unique non-zero entry of $e$ as well as deleting the column $e$, we obtain a suitable representation of our minor. It now follows that if $N$ is any minor of $M$, then $N$ is also $\mathbb{F}$-representable; the class of $\mathbb{F}$-representable matroids is minor-closed. Moreover, a representation for $N$ can be found algebraically by starting with a representation of $M$ and performing a finite sequence of pivots, row deletions and columns deletions. We shall revisit this in Section 3.5.

\subsection{Rota's Conjecture}

The class of matroids is partially ordered under the property of being a minor; as can easily be deduced from the definitions in Section 2.6. Also, as discussed above, the class of $\mathbb{F}$-representable matroids is minor-closed. Now if $M$ is a matroid with $E(M)<4$, it is a simple exercise to show that $M$ is representable over every field. However, as we saw in Section 2.2, there exist matroids which are representable over some fields but not others, as well as 
matroids which are not representable whatsoever. We look to explore the threshold between representability and non-representability. In particular, as the class of $\mathbb{F}$-representable matroids is minor-closed, we can study those matroids which are minor minimal non-representable matroids over $\mathbb{F}$. Such minor minimal non-representable matroids are called excluded minors for $\mathbb{F}$. The following result; due to Tutte [14], gives a complete classification of those matroids which are representable over $G F(2)$ in terms of excluded minors. The matroid $U_{2,4}$ was introduced in Example 2.2.3.

Theorem 2.7.1. A matroid is representable over $G F(2)$ if and only if it has no minor isomorphic to $U_{2,4}$.

The above is an example of an excluded minor theorem. Such a theorem gives a complete classification of a minor-closed class of matroids with respect to the collection of excluded minors for that class. The following result; due to Lazarson [7], reveals that for infinite fields, excluded minor theorems need not be as elegant as Theorem 2.7.1.

Theorem 2.7.2. If $\mathbb{F}$ is a field of characteristic zero, then the set of excluded minors for $\mathbb{F}$-representability is infinite.

However, this is in stark contrast to what is generally believed to be true for finite fields. The following conjecture of Rota [12] is perhaps the most well known conjecture in matroid theory.

Conjecture 2.7.3. (Rota's Conjecture) For any finite field $\mathbb{F}$, the complete set of excluded minors for $\mathbb{F}$-representability is finite.

Proofs that Rota's conjecture is valid in the three element field were first published independently by Bixby [2] and Seymour [13]. The set of excluded minors for $G F(3)$ was found to be as follows:

Theorem 2.7.4. A matroid is representable over $G F(3)$ if and only if it has no minor isomorphic to any of the matroids $U_{2,5}, U_{3,5}, F_{7}$, or $F_{7}^{*}$.

Validity of the conjecture over $G F(4)$ was confirmed by Geelen, Gerards, and Kapoor [4]:

Theorem 2.7.5. A matroid is representable over $G F(4)$ if and only if it has no minor isomorphic to any of the matroids $U_{2,6}, U_{4,6}, P_{6}, F_{7}^{-},\left(F_{7}^{-}\right)^{*}, P_{8}$, or $P_{8}^{\prime \prime}$. 
However, for all finite fields with size exceeding four, Rota's conjecture remains unresolved. Naturally, in seeking a resolution of the conjecture, one would hope to narrow the spectrum of candidates for excluded minor status over $G F(q)$. One simple observation we can make from the above results is that given an arbitrary matroid $M$, key structural information on $M$ can be obtained by considering the minors of $M$. Taking some finite field $G F(q)$, this methodology can now be applied directly to the excluded minors for $G F(q)$. We should be able to shrink our list of potential excluded minors for $G F(q)$ by looking at the necessary structural conditions imposed by the minors of such potential excluded minors. In particular, supposing that $M$ is a potential excluded minor for $G F(q)$, any proper minor $N$ of $M$ is representable over $G F(q)$. Supposing that $N_{1}, \ldots, N_{k}, N_{k+1}=M$ is some ordered sequence of minors of $M$; where $N_{i}$ is a proper minor of $N_{i+1}$ for $i \in\{1, \ldots, k\}$, we know that there exists some matrix representation over $G F(q)$ for $N_{1}, \ldots, N_{k}$. We can therefore examine the representations themselves and hope to use techniques from linear algebra to place necessary conditions on $M$ through inductive methods. In order to do this, we require a sense of consistency when talking about matroid representations. Loosely speaking, one would ideally want the statement 'the representable matroid $N^{\prime}$ is a minor of the representable matroid $N$ ' to correspond with the statement 'a representation of the matroid $N^{\prime}$ can be obtained from a representation of the matroid $N$ through standard algebraic operations" Unfortunately, this is not quite the case.

We noted above that if a matroid $M$ is representable over $\mathbb{F}$, with some representation $A$, then $M$ is invariant under applying standard row operations to $A$, as well as the scaling and interchanging of columns within $A$. The fact that one may also add and/or remove rows of zeros to/from $A$, as well as apply some automorphism of the field $\mathbb{F}$; neither of which alters $M[A]$, leads us to the following definition.

Let $M$ be some matroid which is representable over $\mathbb{F}$, and let $A_{1}$ and $A_{2}$ be matrices; each giving a representation of $M$ over $\mathbb{F}$. If $A_{2}$ can be obtained from $A_{1}$ through a sequence of standard row operations, column interchanges, column scalings, addition/removal of rows of zeros, and automorphisms of $\mathbb{F}$, then the representations $A_{1}$ and $A_{2}$ are said to be equivalent. If all representations of $M$ are equivalent, then we say that $M$ is uniquely representable.

The verifications of Rota's conjecture for $G F(2), G F(3)$ and $G F(4)$ all 
rely heavily on the fact that for these small fields, the key classes of matroids that need to be considered are classes of uniquely representable matroids. However, Oxley, Vertigan, and Whittle [11] showed that certain classes of matroids representable over $G F(5)$ may have as many as six inequivalent representations, while for finite fields containing greater than five elements, the number of possible inequivalent representations of a matroid can be unbounded. This presents a major obstacle on the road toward a resolution of Rota's conjecture.

This obstacle led Whittle [17] to develop the notion of a stabilizer for a class of matroids. Without indulging deeply into the details of defining a general stabilizer here, essentially, a stabilizer for the class of $G F(q)$ representable matroids is a $G F(q)$-representable matroid $N$ such that if $M$ is a 3-connected $G F(q)$-representable matroid which contains $N$ as a minor, then any representation of $M$ is uniquely determined by a representation of $N$. Therefore, if we are to use representations as an inductive tool for examining properties of matroids, we need to find stabilizers for classes of matroids, study matroids which contain these stabilizers, and, in particular, we need to restrict out attention to matroids which are 3-connected. In what follows, we examine this notion of 3-connectivity. 


\section{Chapter 3}

\section{Connectivity}

\subsection{Introduction}

A minor $N$ of a matroid $M$ is, by definition, obtained through applying a sequence of deletions and contractions to the matroid $M$. So if we know that $M$ is 3-connected, and we want to study minors of $M$ that are also 3-connected, we naturally want to know when the fundamental minors $M \backslash e$ and $M / e$ are also 3-connected for particular $e \in E(M)$.

For example, it is a straightforward result that any excluded minor $M$ for $G F(q)$ must be 3-connected. Now, as discussed above, if we want to deduce information about $M$ by examining representations of its minors, then it would certainly help if these minors were uniquely representable; and for that, we require them to be 3 -connected. Now if $N$ is a minor of $M$, then there is a sequence of minors $N=N_{1}, N_{2}, \ldots, N_{k}, N_{k+1}=M$ of $M$ where for $i \in$ $\{1, \ldots, k\}$, there exists some $e_{i} \in E(M)$ such that either $N_{i}=N_{i+1} \backslash e_{i}$ or $N_{i}=$ $N_{i+1} / e_{i}$. Therefore if we can deduce exactly when deleting or contracting an element from a 3-connected matroid results in another 3-connected matroid, we may be able to obtain a sequence of uniquely representable matroids $N=N_{1}, N_{2}, \ldots, N_{k}, N_{k+1}=M$; which would be helpful in an attempt to deduce structure of $M$. Firstly, some definitions:

Definition 3.1.1. Let $M$ be a matroid on a ground set $E$. The connectivity function $\lambda$ of $M$ is defined on all subsets $X$ of $E$ by

$$
\lambda(X)=r(X)+r(E-X)-r(M) .
$$


When dealing with multiple matroids, we shall write $\lambda_{M}$ to mean the connectivity function of the matroid $M$. It is clear that $\lambda(X)=\lambda(E-X)$ for any $X \subseteq E$. A subset $X$ or a partition $(X, E-X)$ of $M$ is $k$-separating if $\lambda(X) \leq k-1$. A $k$-separating partition $(X, E-X)$ is a $k$-separation if both $|X| \geq k$ and $|E-X| \geq k$. A $k$-separating set $X$, or a $k$-separating partition $(X, E-X)$, or a $k$-separation $(X, E-X)$ is exact if $\lambda(X)=k-1$. A matroid is said to be $n$-connected provided it has no $k$-separations for all $k<n$. When a matroid is 2-connected, we simply say that it is connected.

Lemma 3.1.2. Let $M$ be a matroid. Then $\lambda_{M}=\lambda_{M^{*}}$. That is, for all $X \subseteq E(M), \lambda_{M}(X)=\lambda_{M^{*}}(X)$.

Proof. Suppose $X \subseteq E(M)$. Then, by Lemma 2.5.3,

$$
\begin{aligned}
\lambda_{M}(X) & =r(X)+r(E-X)-r(M) \\
& =r(X)+r^{*}(X)-|X| \\
& =r^{*}(E-X)+r(M)-|E-X|+r^{*}(X)-|X| \\
& =r^{*}(X)+r^{*}(E-X)-(|E(M)|-r(M)) \\
& =r^{*}(X)+r^{*}(E-X)-r^{*}(M)=\lambda_{M^{*}}(X) .
\end{aligned}
$$

The above result, as well as the next two, will be used freely.

Corollary 3.1.3. Let $M$ be a matroid. Then $M$ is $n$-connected if and only if $M^{*}$ is $n$-connected.

Proof. This follows immediately from Lemma 3.1.2.

Lemma 3.1.4. Let $M$ be an n-connected matroid with $|E(M)| \geq 4$ and $e \in E(M)$. Then both $M \backslash e$ and $M / e$ are $(n-1)$-connected.

Proof. Suppose $N \in\{M \backslash e, M / e\}$ and that $X$ and $Y$ are disjoint subsets of $E(M)$ whose union is $E(M)-\{e\}$. Then by Lemma 2.6.3, $r_{N}(X) \in\left\{r_{M}(X), r_{M}(X)-1\right\}, r_{N}(Y) \in\left\{r_{M}(Y), r_{M}(Y)-1\right\}$, and $r(N) \in$ $\{r(M), r(M)-1\}$. So certainly, $\lambda_{N}(X)=r_{N}(X)+r_{N}(Y)-r(N) \in$ $\left\{\lambda_{M}(X), \lambda_{M}(X)-1, \lambda_{M}(X)-2\right\}$, and the only permutation that gives $\lambda_{N}(X)=\lambda_{M}(X)-2$ is where $r(N)=r(M), r_{N}(X)=r_{M}(X)-1$, and 
$r_{N}(Y)=r_{M}(Y)-1$. But in this case, the fact that $r_{N}(X)=r_{M}(X)-1$ implies that $N=M / e$ and $e$ is not a loop of $M$; contradicting the necessary requirement that $r(N)=r(M)$. Therefore $\lambda_{N}(X) \in\left\{\lambda_{M}(X), \lambda_{M}(X)-1\right\}$. As $(X, Y)$ was an arbitrary partition of $E(N)$, it follows that $N$ has no $(n-k)$-separations for all $k \geq 2$. Therefore $N$ is $(n-1)$-connected.

Lemma 3.1.5. Let $M$ be a matroid on a ground set $E$ and suppose $X, Y \subseteq E$. Then $\lambda(X \cup Y)+\lambda(X \cap Y) \leq \lambda(X)+\lambda(Y)$.

Proof. With the help of Lemmas 2.3.2 and 2.5.3, we have

$$
\begin{aligned}
& \lambda(X \cup Y)+\lambda(X \cap Y) \\
= & r(X \cup Y)+r(X \cap Y)+r(E-(X \cup Y))+r(E-(X \cap Y))-2 r(M) \\
\leq & r(X)+r(Y)+r(E-(X \cup Y))+r(E-(X \cap Y))-2 r(M) \\
= & r(X)+r(Y)+r^{*}(X \cup Y)+r^{*}(X \cap Y)-|X \cup Y|-|X \cap Y| \\
= & r(X)+r(Y)+r^{*}(X \cup Y)+r^{*}(X \cap Y)-|X|-|Y| \\
\leq & r(X)+r(Y)+r^{*}(X)+r^{*}(Y)-|X|-|Y| \\
= & r(X)+r(Y)+r(E-X)+r(E-Y)-2 r(M)=\lambda(X)+\lambda(Y) .
\end{aligned}
$$

We shall often say by submodularity of $\lambda$ to mean an application of Lemma 3.1.5.

\subsection{Various Lemmas on 3-connectivity}

We now present a series of results relating specifically to 3-connectivity. The first of these is used repeatedly throughout the thesis. When applying it, we shall often simply use the phrase by uncrossing.

Lemma 3.2.1. Let $M$ be a 3-connected matroid, and let $X$ and $Y$ be 3separating subsets of $E(M)$. Then:

(i) if $|X \cap Y| \geq 2, X \cup Y$ is 3-separating, and

(ii) if $|E(M)-(X \cup Y)| \geq 2, X \cap Y$ is 3-separating. 
Proof. Suppose $|X \cap Y| \geq 2$. Then by submodularity of $\lambda$, we have $\lambda(X \cup$ $Y) \leq \lambda(X)+\lambda(Y)-\lambda(X \cap Y) \leq 4-\lambda(X \cap Y) \leq 2$, so that $X \cup Y$ is 3-separating. Similarly, supposing that $|E(M)-(X \cup Y)| \geq 2$, it follows that $\lambda(X \cap Y) \leq \lambda(X)+\lambda(Y)-\lambda(X \cup Y) \leq 4-\lambda(E-(X \cup Y)) \leq 2$.

Lemma 3.2.2. Let $M$ be a 3-connected matroid. If $L$ is a line of $M$ with $|L| \geq 4$, then $M \backslash x$ is 3-connected for all $x \in L$.

Proof. Suppose $x \in L$ and $M \backslash x$ is not 3-connected. Then there exists a 2-separation $(P, Q)$ of $M \backslash x$. Without loss of generality, $|L \cap P| \geq 2$. But now $x \in \operatorname{cl}(P)$, so that $\lambda_{M \backslash x}(P)=\lambda_{M}(P \cup\{x\})$; giving a contradictory 2-separation $(P \cup\{x\}, Q)$ of the 3-connected matroid $M$.

Lemma 3.2.3. Let $M$ be a 3-connected matroid. If $\Gamma$ is a coline of $M$ with $|\Gamma| \geq 4$, then $M / y$ is 3-connected for all $y \in \Gamma$.

Proof. Let $\Gamma$ be a coline of $M$ with $|\Gamma| \geq 4$. Then $\Gamma$ is a line of the 3 connected matroid $M^{*}$. So by Lemma 3.2.2, $M^{*} \backslash y$ is 3-connected for all $y \in \Gamma$. Therefore $\left(M^{*} \backslash y\right)^{*}=M / y$ is 3 -connected for all $y \in \Gamma$.

Lemma 3.2.4. Let $M$ be a 3-connected matroid. Let $\Gamma \subseteq E(M)$ be a coline of $M$. Then provided $|E(M)-\Gamma| \geq 2, r(\Gamma)=|\Gamma|$.

Proof. Let $\Gamma$ be a coline of $M$. Using Lemma 2.5.3 and the fact that $M$ is 3-connected, we have

$$
\begin{aligned}
& \lambda(\Gamma)=r(\Gamma)+r(E(M)-\Gamma)-r(M) \geq 2 \\
& \quad \Longrightarrow r(\Gamma)+2+r(M)-|\Gamma|-r(M) \geq 2 \\
& \quad \Longrightarrow r(\Gamma) \geq|\Gamma|
\end{aligned}
$$

So that $r(\Gamma)=|\Gamma|$.

Lemma 3.2.5. Let $M$ be a 3-connected matroid, and let $B$ be a basis of $M$. If $\Gamma_{1}, \Gamma_{2}$ are 4-point colines of $M$ with $\left|\Gamma_{1} \cap B\right|=\left|\Gamma_{2} \cap B\right|=2$, then, if $\Gamma_{1} \cap \Gamma_{2} \neq \emptyset, \Gamma_{1} \cap \Gamma_{2} \not \subset B$.

Proof. Let $\Gamma_{1}, \Gamma_{2}$ be 4-point colines of $M$ such that $\left|\Gamma_{1} \cap B\right|=\left|\Gamma_{2} \cap B\right|=2$ and $\Gamma_{1} \cap \Gamma_{2} \neq \emptyset$. As $M$ is 3-connected, it follows from Lemma 2.3.2 that $r^{*}\left(\Gamma_{1} \cup \Gamma_{2}\right) \in\{2,3\}$. If $\Gamma_{1}=\Gamma_{2}$, then the result is immediate, so assume that 
$\Gamma_{1}$ and $\Gamma_{2}$ are distinct. Suppose that $\Gamma_{1} \cap \Gamma_{2} \subseteq B$. If $\left|\Gamma_{1} \cap \Gamma_{2}\right| \geq 2$, then by Lemma 2.5.7, $\Gamma_{1} \cup \Gamma_{2}$ is a coline containing at least five elements. But then $\left|\left(\Gamma_{1} \cup \Gamma_{2}\right) \cap(E(M)-B)\right| \geq 3$, so that $r^{*}\left(\Gamma_{1} \cup \Gamma_{2}\right) \geq 3$; contradicting the fact that $\Gamma_{1} \cup \Gamma_{2}$ is a coline. Otherwise, $\left|\Gamma_{1} \cap \Gamma_{2}\right|=1$; implying that $\left|\left(\Gamma_{1} \cup \Gamma_{2}\right) \cap(E(M)-B)\right| \geq 4$; which contradicts the fact $r^{*}\left(\Gamma_{1} \cup \Gamma_{2}\right) \in\{2,3\}$. Therefore $\Gamma_{1} \cap \Gamma_{2} \nsubseteq B$.

Lemma 3.2.6. Let $(X, Y)$ be an exact 3-separation of a 3-connected matroid $M$. If $X$ is independent, then $X$ is a coline of $M$.

Proof. Suppose $X$ is independent. Then, by Lemma 2.5.3,

$$
\begin{aligned}
r^{*}(X) & =|X|-r(M)+r(E(M)-X) \\
& =|X|-r(M)+r(Y) \\
& =|X|-r(M)+r(M)+2-r(X) \\
& =|X|+2-r(X)=2 .
\end{aligned}
$$

So that $X$ is a coline of $M$.

We shall write $x \in \mathrm{cl}^{(*)}(Y)$ to mean that $x \in \operatorname{cl}(Y)$ or $x \in \mathrm{cl}^{*}(Y)$.

Lemma 3.2.7. Let $X$ be an exactly 3-separating set in a 3-connected matroid $M$, and suppose that $r \in E(M)-X$. Then $X \cup\{r\}$ is 3-separating if and only if $r \in \mathrm{cl}^{(*)}(X)$.

Proof. We have $\lambda(X)=r(X)+r(E(M)-X)-r(M)=2$. Now Lemma 2.5.4 together with some simple arithmetic reveals that $\lambda(X \cup\{r\}) \leq 2$ iff $r(X \cup$ $\{r\})+r(E(M)-(X \cup\{r\}))-r(M) \leq 2$; iff $r(X \cup\{r\})=r(X)$, or $r(X \cup\{r\})=$ $r(X)+1$ and $r(E(M)-(X \cup\{r\}))=r(E(M)-X)-1$; iff $r \in \operatorname{cl}(X)$ or $r \notin \operatorname{cl}(E(M)-X)$; iff $r \in \operatorname{cl}(X)$ or $r \in \mathrm{cl}^{*}(X)$; iff $r \in \mathrm{cl}^{(*)}(X)$.

Lemma 3.2.8. Let $(X, Y)$ be an exactly 3-separating partition of a 3connected matroid $M$. Suppose $|X| \geq 3$ and $x \in X$. Then:

(i) $x \in \mathrm{cl}^{(*)}(X-\{x\})$; and

(ii) $(X-\{x\}, Y \cup\{x\})$ is exactly 3-separating if and only if $x$ is in exactly one of $\operatorname{cl}(X-\{x\}) \cap \operatorname{cl}(Y)$ and $\mathrm{cl}^{*}(X-\{x\}) \cap \mathrm{cl}^{*}(Y)$. 
Proof. Assume that $x \notin \mathrm{cl}^{*}(X-\{x\})$. Then $x \in \operatorname{cl}(Y)$ by Lemma 2.5.4, so $r(Y)=r(Y \cup\{x\})$. Now suppose that $x \notin \operatorname{cl}(X-\{x\})$. Then $r(X)=$ $r(X-\{x\})+1$, and therefore

$$
\begin{aligned}
& r(X)+r(Y)-r(M)=2 \\
\Longrightarrow & r(X-\{x\})+1+r(Y \cup\{x\})-r(M)=2 \\
\Longrightarrow & r(X-\{x\})+r(Y \cup\{x\})-r(M)=1
\end{aligned}
$$

But $|X-\{x\}| \geq 2$ so that $(X-\{x\}, Y \cup\{x\})$ is a contradictory 2-separation of $M$. Therefore $x \notin \mathrm{cl}^{*}(X-\{x\}) \Longrightarrow x \in \operatorname{cl}(X-\{x\})$. Thus $x \in \mathrm{cl}^{(*)}(X-$ $\{x\})$; completing the proof of (i). Now, using Lemma 2.5.4, $\lambda(X-\{x\})=$ 2 iff $r(X-\{x\})+r(Y \cup\{x\})-r(M)=2$; iff $r(X-\{x\})=r(X)$ and $r(Y \cup\{x\})=r(Y)$, or $r(X-\{x\})=r(X)-1$ and $r(Y \cup\{x\})=r(Y)+1$; iff $x \in \operatorname{cl}(X-\{x\}) \cap \operatorname{cl}(Y)$, or $x \notin \operatorname{cl}(X)$ and $x \notin \operatorname{cl}(Y)$; iff $x \in \operatorname{cl}(X-\{x\}) \cap \operatorname{cl}(Y)$ or $x \in\left(\operatorname{cl}^{*}(X) \cap \operatorname{cl}^{*}(Y)\right)-\operatorname{cl}(X)$; iff $x$ is in exactly one of $\operatorname{cl}(X-\{x\}) \cap \operatorname{cl}(Y)$ and $\operatorname{cl}^{*}(X) \cap \mathrm{cl}^{*}(Y)$.

\subsection{Vertical \& Cyclic Separations}

The following types of $k$-separations will play a critical role throughout the remainder of this thesis.

Definition 3.3.1. A $k$-separation $(P, E-P)$ of a matroid $M=(E, \mathcal{I})$ is vertical if both $r(P) \geq k$ and $r(E-P) \geq k$. A $k$-separation $(P, E-P)$ is cyclic if both $P$ and $E-P$ contain circuits of $M$. Let $(X,\{a\}, Y)$ be a partition of $E$. If $(X \cup\{a\}, Y)$ and $(X, Y \cup\{a\})$ are both vertical 3-separations of $M$ and $a \in \operatorname{cl}(X) \cap \operatorname{cl}(Y)$, then we say that $(X,\{a\}, Y)$ is a vertical 3-separation of $M$. If $(X \cup\{a\}, Y)$ and $(X, Y \cup\{a\})$ are both cyclic 3-separations of $M$ and $a \in \mathrm{cl}^{*}(X) \cap \mathrm{cl}^{*}(Y)$, then we say that $(X,\{a\}, Y)$ is a cyclic 3-separation of $M$.

The next two results are used freely throughout.

Lemma 3.3.2. Let $M$ be a k-connected matroid. A k-separation $(X, E(M)-$ $X)$ of $M$ is a vertical $k$-separation of $M$ if and only if it is a cyclic $k$ separation of $M^{*}$. 
Proof. Suppose that $(X, E(M)-X)$ is a vertical $k$-separation of $M$. Then $\lambda(X)=k-1$ because $M$ is $k$-connected. If $X$ is coindependent, then $E(M)-X$ is spanning by Lemma 2.5.2. But then $r(X)=k-1+r(M)-$ $r(E(M)-X)=k-1$ which contradicts the fact that $(X, E(M)-X)$ is vertical. Therefore $X$ must contain a cocircuit. Similarly, $E(M)-X$ must also contain a cocircuit. Thus, both $X$ and $E(M)-X$ contain circuits of $M^{*}$ and $(X, E(M)-X)$ is a cyclic $k$-separation of $M^{*}$. The converse follows in the same manner.

Corollary 3.3.3. Let $M$ be a k-connected matroid. A partition $(X,\{a\}, Y)$ of $M$ is a vertical $k$-separation of $M$ if and only if it is a cyclic $k$-separation of $M^{*}$.

Proof. This follows immediately from Lemma 3.3.2.

Lemma 3.3.4. Let $(P, Q)$ be a cyclic $k$-separation of a matroid $M$. If a $\in$ $\operatorname{cl}^{*}(P) \cap Q$, then $(P \cup\{a\}, Q-\{a\})$ is also a cyclic k-separation of $M$.

Proof. Suppose $a \in \mathrm{cl}^{*}(P) \cap Q$. If $Q-\{a\}$ is independent, then $a \in \operatorname{cl}(Q-\{a\})$ because $Q$ contains a circuit. But this contradicts Lemma 2.5.4. Therefore $Q-\{a\}$ must contain a circuit.

Lemma 3.3.5. Let $M$ be a 3-connected matroid with $c \in E(M)$. If $(P,\{c\}, Q)$ is a cyclic 3-separation of $M$, then $\left(\mathrm{cl}^{*}(P)-\{c\},\{c\}, Q-\mathrm{cl}^{*}(P)\right)$ is also a cyclic 3-separation of $M$.

Proof. This follows by repeated application of Lemma 3.3.4.

Lemma 3.3.6. Let $M$ be a 3-connected matroid with $b \in E(M)$. If $(X,\{b\}, Y)$ is a vertical 3-separation of $M$, then $(\operatorname{cl}(X)-\{b\},\{b\}, Y-\operatorname{cl}(X))$ is also a vertical 3-separation of $M$.

Proof. Suppose $e \in \operatorname{cl}(X) \cap Y$. Then $e \in \operatorname{cl}(X \cup\{b\})$, so that by Lemmas 2.5.4 and 3.2.8, $e \in \operatorname{cl}(Y-\{e\})$. Now both of $(X \cup\{b, e\}, Y-\{e\})$ and $(X \cup$ $\{e\}, Y \cup\{b\})$ are exactly 3-separating by Lemma 3.2.8, and it follows that $(X \cup\{e\},\{b\}, Y-\{e\})$ is a vertical 3-separation of $M$. By an iteration of this argument, we deduce that $(\operatorname{cl}(X)-\{b\},\{b\}, Y-\operatorname{cl}(X))$ is also a vertical 3-separation of $M$. 
Lemma 3.3.7. Suppose $(X,\{a\}, Y)$ is a vertical or cyclic 3-separation of a 3-connected matroid $M$. Then $\left(\operatorname{cl}(X) \cap \mathrm{cl}^{*}(X)\right)-X=\emptyset$.

Proof. If there exists some $e \in \operatorname{cl}(X)-X$, then $e \notin \mathrm{cl}^{*}(Y-\{a, e\})$ by Lemma 2.5.4. Therefore $e \in \operatorname{cl}(Y-\{a, e\})$ by Lemma 3.2.8, and so, by

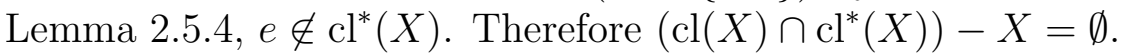

Definition 3.3.8. A parallel class of a matroid $M$ is a maximal subset of $E(M)$ such that any two members form a parallel pair. A series class of $M$ is a parallel class of $M^{*}$.

It follows that any 3-connected matroid $M$ does not contain any parallel or series classes of size greater than one, for if it did, then; up to duality, $M$ would contain a parallel class $P$ of size two or greater, giving a contradictory 2-separation $(P, E(M)-P)$ of $M$. This fact will be used implicitly throughout.

Definition 3.3.9. Let $M$ be a matroid. The simplification of $M$; denoted si $(M)$, is the minor of $M$ which is obtained by deleting all loops of $M$ and all but one element from each parallel class of $M$. The cosimplification of $M$; denoted $\operatorname{co}(M)$, is defined by $\operatorname{co}(M)=\left(\operatorname{si}\left(M^{*}\right)\right)^{*}$.

When deleting and contracting elements from a 3 -connected matroid $M$; resulting in a minor $N$ of $M$, it is often necessary to restrict our attention to the matroids $\operatorname{si}(N)$ and $\operatorname{co}(N)$ in order to maintain 3-connectivity. For example, if $r(M) \geq 3$ and $L \subset E(M)$ is a line containing more than two points, then contracting any element $e \in L$ gives a 2-separation $(L-\{e\}, E(M)-L)$ of the matroid $M / e$ because $L-\{e\}$ becomes a parallel class in $M / e$ of size at least two. But if we move to the matroid $\operatorname{si}(M / e)$, this parallel class becomes a single element, which removes the trivial 2-separation that arose upon contraction. This allows us to better examine the global connectivity structure of the matroid $M / e$, and it may be that we have now found a minor si $(M / e)$ of $M$ which is indeed 3-connected. If $\operatorname{si}(N)$ is 3 -connected, then we say than $N$ is 3-connected up to parallel classes. Similarly, if $\operatorname{co}(N)$ is 3-connected, we say that $N$ is 3 -connected up to series classes.

Lemma 3.3.10. A 2-separation $(X, Y)$ of a connected matroid $M$ is cyclic if and only if neither $X$ nor $Y$ is a series class. 
Proof. A 2-separation $(X, Y)$ is not cyclic in $M$ iff it is not vertical in $M^{*}$; iff either $r^{*}(X)=1$ or $r^{*}(Y)=1$; iff $X$ or $Y$ is a parallel class in $M^{*}$; iff $X$ or $Y$ is a series class in $M$.

Lemma 3.3.11. Let $M$ be a 3-connected matroid. If there exists some $c \in$ $E(M)$ such that $\operatorname{co}(M \backslash c)$ is not 3-connected, then there exists a cyclic 2separation of $M \backslash c$.

Proof. Suppose that $\operatorname{co}(M \backslash c)$ is not 3-connected for some $c \in E(M)$. Then there exists a 2-separation $\left(X^{\prime}, Y^{\prime}\right)$ of $\operatorname{co}(M \backslash c)$. By Lemma 3.3.10, $\left(X^{\prime}, Y^{\prime}\right)$ is cyclic. Therefore $\left(X^{\prime}, Y^{\prime}\right)$ is a vertical 2-separation of $(\operatorname{co}(M \backslash c))^{*}=$ $\operatorname{si}\left(M^{*} / c\right)$. Let $S=E\left(M^{*} / c\right)-E\left(\operatorname{si}\left(M^{*} / c\right)\right)$. Then by definition of simplification, for all $s \in S, s$ is contained in exactly one of $\mathrm{cl}_{M^{*} / c}\left(X^{\prime}\right)$ and $\mathrm{cl}_{M^{*} / c}\left(Y^{\prime}\right)$. Let $S_{x}=S \cap \mathrm{cl}_{M^{*} / c}\left(X^{\prime}\right)$ and $S_{y}=S \cap \mathrm{cl}_{M^{*} / c}\left(Y^{\prime}\right)$. Taking $X=X^{\prime} \cup S_{x}$ and $Y=Y^{\prime} \cup S_{y}$, it then follows directly from the definition of $k$-separation that $\left(X \cup S_{x}, Y \cup S_{y}\right)$ is a vertical 2-separation of $M^{*} / c$; hence a cyclic 2-separation of $\left(M^{*} / c\right)^{*}=M \backslash c$.

The following two results are used freely and extensively throughout the remainder of this thesis.

Lemma 3.3.12. Let $M$ be a 3-connected matroid with $b \in E(M)$. If $\operatorname{si}(M / b)$ is not 3-connected, then there exists a vertical 3-separation $(X,\{b\}, Y)$ of $M$.

Proof. Suppose si $(M / b)$ is not 3-connected. Then there exists a 2-separation $(X, Y)$ of $M / b$ such that $(X \cap E(\operatorname{si}(M / b)), Y \cap E(\operatorname{si}(M / b)))$ is a 2-separation of $\operatorname{si}(M / b)$. We have $r_{M / b}(X)+r_{M / b}(Y)-r(M / b)=1$, so by Lemma 2.6.5, $r_{M}(X \cup\{b\})+r_{M}(Y \cup\{b\})-r(M)=2$. Now if $b \notin \operatorname{cl}(X)$, then $r_{M}(X)+$ $r_{M}(Y \cup\{b\})-r(M)=1$; which contradicts the fact that $M$ is 3-connected. So $b \in \operatorname{cl}(X)$. Similarly, $b \in \operatorname{cl}(Y)$. As $r_{M / b}(X) \geq 2$, we have $r_{M}(X)=$ $r_{M}(X \cup\{b\}) \geq 3$. Similarly, $r_{M}(Y) \geq 3$. We conclude that $(X,\{b\}, Y)$ is a vertical 3-separation of $M$.

Lemma 3.3.13. Let $M$ be a 3-connected matroid with $c \in E(M)$. If $\operatorname{co}(M \backslash c)$ is not 3-connected, then there exists a cyclic 3-separation $(P,\{c\}, Q)$ of $M$.

Proof. This follows immediately as the dual of Lemma 3.3.12. 


\subsection{Further Results on Connectivity}

We conclude this chapter with several results of importance to following chapters.

Lemma 3.4.1. Let $A$ be a cocircuit of a 3-connected matroid $M$ where $r(A)=3$ and $e \in A$. If, for some set $\{p, q, r\} \subseteq A-\{e\}, p, q$ and $r$ are each contained in distinct parallel classes of $M / e$, then $\operatorname{si}(M / e)$ is 3-connected.

Proof. Suppose $\{p, q, r\} \subseteq A-\{e\}$ where $p, q$ and $r$ are each contained in a distinct parallel class of $M / e$. Assume that $\operatorname{si}(M / e)$ is not 3-connected. Then $M$ has a vertical 3 -separation $(X,\{e\}, Y)$. It follows that $(X, Y)$ is a vertical 2-separation of $M / e$. Without loss of generality, $|X \cap\{p, q, r\}| \geq 2$, so we may assume that $\{p, q\} \subseteq X$. Now, as $r_{M / e}(A-\{e\})=2$, we have $r_{M / e}(X \cap(A-\{e\}))=2$. By the dual of Lemma 3.3.4, it follows that $\left(\mathrm{cl}_{M / e}(X), Y-\operatorname{cl}_{M / e}(X)\right)$ is a vertical 2-separation of $M / e$ where $A-\{e\} \subseteq$ $\mathrm{cl}_{M / e}(X)$. Then $Y-\mathrm{cl}_{M / e}(X)$ is contained in the hyperplane $E(M)-A$, which implies that $r_{M}\left(Y-\operatorname{cl}_{M / e}(X)\right)=r_{M / e}\left(Y-\operatorname{cl}_{M / e}(X)\right)$. But then

$$
\begin{aligned}
\lambda_{M}\left(Y-\operatorname{cl}_{M / e}(X)\right) & =r_{M}\left(Y-\operatorname{cl}_{M / e}(X)\right)+r_{M}\left(\operatorname{cl}_{M / e}(X) \cup\{e\}\right)-r(M) \\
& =r_{M / e}\left(Y-\operatorname{cl}_{M / e}(X)\right)+r_{M / e}\left(\operatorname{cl}_{M / e}(X)\right)-r(M / e) \\
& =\lambda_{M / e}\left(Y-\operatorname{cl}_{M / e}(X)\right) \leq 1
\end{aligned}
$$

So that $\left(\mathrm{cl}_{M / e}(X) \cup\{e\}, Y-\mathrm{cl}_{M / e}(X)\right)$ is a 2-separation of $M$; contradicting the fact that $M$ is 3 -connected. Therefore $\operatorname{si}(M / e)$ must be 3 -connected.

The following result; known as Bixby's Lemma [3], shows that up to parallel and series classes, every element of a 3-connected matroid can either be contracted or deleted to give a minor which remains 3-connected.

Lemma 3.4.2. Let e be an element of a 3-connected matroid $M$. Then either $\operatorname{si}(M / e)$ or $\operatorname{co}(M \backslash e)$ is 3-connected.

Proof. Suppose that neither $\operatorname{si}(M / e)$ nor $\operatorname{co}(M \backslash e)$ are 3-connected. Let $(X,\{e\}, Y)$ and $(S,\{e\}, T)$ be vertical and cyclic 3 -separations of $M$ respectively.

3.4.2.1. For all $Z \in\{X \cap S, X \cap T, Y \cap S, Y \cap T\}, Z \neq \emptyset$. 
Proof. Suppose $X \cap S=\emptyset$. Then $X \subseteq T$. Therefore $e \in \operatorname{cl}(T) \cap \operatorname{cl}^{*}(T)$; but this contradicts Lemma 3.3.7. Similarly, each of $X \cap T, Y \cap S$, and $Y \cap T$ are non-empty.

3.4.2.2. For all $Z \in\{X \cap S, X \cap T, Y \cap S, Y \cap T\}, \lambda(Z) \leq 2$.

Proof. $\lambda(X)=\lambda(S)=2$. By 3.4.2.1, $|E-(X \cup S)|=|(Y \cap T) \cup\{e\}| \geq 2$, so that by uncrossing, $\lambda(X \cap S) \leq 2$. Similarly, each of $X \cap T, Y \cap S$, and $Y \cap T$ are 3 -separating.

3.4.2.3. $|X \cap S| \geq 2$ and $|Y \cap T| \geq 2$

Proof. Suppose that $|X \cap S|=1$. Then $|X \cap T| \geq 2$ and $|Y \cap S| \geq 2$ because $r(X) \geq 3$ and $S$ contains a circuit. So by 3.4.2.2, $\lambda(X \cap T)=2$ as $M$ is 3-connected. Furthermore, $\lambda(X \cup\{e\})=\lambda(T \cup\{e\})=2$ and $|E-((X \cup\{e\}) \cup(T \cup\{e\}))|=|Y \cap S| \geq 2$, so that, by uncrossing, $\lambda((X \cup\{e\}) \cap$ $(T \cup\{e\}))=\lambda((X \cap T) \cup\{e\})=2$. Now by Lemma 3.2.7, $e \in \mathrm{cl}^{(*)}(X \cap T)$. But $e \in \operatorname{cl}(Y) \cap \operatorname{cl}^{*}(S)$, so $e \in \operatorname{cl}(Y \cup S) \cap \mathrm{cl}^{*}(Y \cup S)$; which contradicts Lemma 2.5.4. Therefore $|X \cap S| \neq 1$, and by 3.4.2.2, $|X \cap S| \geq 2$. Similarly, $|Y \cap T| \geq 2$.

By 3.4.2.2 and 3.4.2.3, $\lambda(X \cap S)=2$. Also by 3.4.2.3, $\mid E-((X \cup\{e\}) \cup(S \cup$ $\{e\}))|=| Y \cap T \mid \geq 2$. Therefore, by uncrossing, $\lambda((X \cap S) \cup\{e\})=2$. Now by Lemma 3.2.7, $e \in \operatorname{cl}(X \cap S)$ or $e \in \operatorname{cl}^{*}(X \cap S)$. But then by Lemma 3.3.3, the first possibility contradicts the fact that $e \in \mathrm{cl}^{*}(T)$; while the second contradicts the fact that $e \in \operatorname{cl}(Y)$. We are left to deduce that at least one of $\operatorname{si}(M / e)$ or $\operatorname{co}(M \backslash e)$ is 3-connected.

Lemma 3.4.3. Let $(X,\{b\}, Y)$ be a vertical 3-separation of a 3-connected matroid $M$. If $z \in X \cap \operatorname{cl}^{*}(Y)$ then either $(X-\{z\},\{b\}, Y \cup\{z\})$ is a vertical 3-separation of $M$ or $r((X-\{z\}) \cup\{b\})=2$.

Proof. Suppose $z \in X$ is such that $z \in \mathrm{cl}^{*}(Y)$. By Lemma 3.2.8, $z \in$ $\mathrm{cl}^{(*)}((X \cup\{b\})-\{z\})$. As $z \in \mathrm{cl}^{*}(Y), z \notin \operatorname{cl}((X \cup\{b\})-\{z\})$ by Lemma 2.5.4. So $z \in \mathrm{cl}^{*}((X \cup\{b\})-\{z\})$. Therefore $(((X \cup\{b\})-\{z\}), Y \cup\{z\})$ is an exactly 3-separating partition of $M$ by Lemma 3.2.8 (ii). If $|X-\{z\}|=2$ and $r((X-\{z\}) \cup\{b\})=3$, then $r(X)=3=r(X \cup\{b\})$, so that $z \in$ $\operatorname{cl}((X-\{z\}) \cup\{b\})$ which; by Lemma 2.5.4, contradicts our assumption that $z \in \mathrm{cl}^{*}(Y)$. Therefore whenever $|X-\{z\}|=2$, it must be that $b \in$ 
$\operatorname{cl}(X-\{z\})$ as required. So assume that $|X-\{z\}| \geq 2$. Now $b \in \mathrm{cl}^{(*)}(X-\{z\})$ by Lemma 3.2.8 (i). As $b \in \operatorname{cl}(Y) \subset \operatorname{cl}(Y \cup\{z\}), b \notin \operatorname{cl}^{*}(X-\{z\})$ by Lemma 2.5.4. So $b \in \operatorname{cl}(X-\{z\})$. Hence, $(X-\{z\}, Y \cup\{b, z\})$ is an exactly 3separating partition of $M$ by Lemma 3.2.8 (ii). Therefore, if $r(X-\{z\}) \geq 3$, then $(X-\{z\},\{b\}, Y \cup\{z\})$ is a vertical 3-separation of $M$. Otherwise, $r(X-\{z\})=r((X-\{z\}) \cup\{b\})=2$.

Theorem 3.4.4. Let $M$ be a 3-connected matroid with $r(M) \geq 4$. Suppose that $C^{*}$ is a rank 3 cocircuit of $M$. If there exists some $c \in C^{*}$ such that $r\left(C^{*}-\{c\}\right)=3$, then $\operatorname{co}(M \backslash c)$ is 3-connected.

Proof. Suppose that $c \in C^{*}$ and $r\left(C^{*}-\{c\}\right)=3$, but $\operatorname{co}(M \backslash c)$ is not 3connected. Then by Lemma 3.3.11, there exists a cyclic 2-separation $(P, Q)$ of $M \backslash c$.

As $C^{*}$ is a cocircuit, we have $r\left(E(M)-C^{*}\right)=r(M)-1$. Hence $\lambda_{M}\left(C^{*}\right)=2$. Noting that $r\left(E(M)-C^{*}\right) \geq 3$ because $r(M) \geq 4$, we have that $\left(C^{*}, E(M)-C^{*}\right)$ is a vertical 3-separation of $M$. For clarity, let $X=C^{*}-\{c\}$, and $Y=E(M)-C^{*}$ so that $(X \cup\{c\}, Y)$ is a 3-separation of $M$.

3.4.4.1. Each of the intersections $X \cap P, X \cap Q, Y \cap P$, and $Y \cap Q$ are non-empty.

Proof. If $X \cap P=\emptyset$, then $X \subseteq Q$. But then $c \in \operatorname{cl}(X) \subseteq \operatorname{cl}(Q)$ so that $(P, Q \cup\{c\})$ is a contradictory 2-separation of $M$. Similarly, $X \cap Q \neq \emptyset$. If $Y \cap P=\emptyset$, then $Y \subseteq Q$. Now $Y$ is a hyperplane of $M$ and $X \cap Q \neq \emptyset$, so that $r(Q)=r(M)$ and $c \in \operatorname{cl}(Q)$; again giving a contradictory 2-separation $(P, Q \cup\{c\})$ of $M$. Similarly, $Y \cap Q \neq \emptyset$.

3.4.4.2. For all $Z \in\{X \cap P, X \cap Q, Y \cap P, Y \cap Q\}, \lambda_{M \backslash c}(Z)=\lambda_{M}(Z)$.

Proof. By hypothesis, $r(M \backslash c)=r(M)$. If $Z \in\{X \cap P, X \cap Q\}$, then by 3.4.4.1 and the fact that $Y$ is a hyperplane, $c \in \operatorname{cl}(E(M)-Z)$. If $Z \in\{Y \cap P, Y \cap Q\}$, then $c \in \operatorname{cl}(X) \subseteq \operatorname{cl}(E(M)-Z)$, and in either case we have $\lambda_{M \backslash c}(Z)=$ $\lambda_{M}(Z)$.

Now, by the submodularity of the connectivity function $\lambda$, we see that $\lambda_{M \backslash c}(X \cap P)+\lambda_{M \backslash c}(X \cup P) \leq \lambda_{M \backslash c}(X)+\lambda_{M \backslash c}(P)=2+1=3$. So, $\lambda_{M \backslash c}(X \cap P)+\lambda_{M \backslash c}(Y \cap Q) \leq \lambda_{M \backslash c}(X)+\lambda_{M \backslash c}(P)=3$. By 3.4.4.2, $\lambda_{M}(X \cap$ $P)+\lambda_{M}(Y \cap Q) \leq 3$. So at least one of $\{X \cap P, Y \cap Q\}$ is 2-separating. As $M$ 
is 3-connected, it then must be the case that at least one of $\{X \cap P, Y \cap Q\}$ has cardinality equal to 1 . Now, applying submodularity of the function $\lambda$ and 3.4.4.2 again to $X \cap Q$, we deduce that at least one of $\{X \cap Q, Y \cap P\}$ has cardinality equal to 1 . We cannot have $|X \cap P|=1=|X \cap Q|$ as $|X| \geq 3$. It also cannot be that $|Y \cap P|=1=|Y \cap Q|$ as $r(Y) \geq 3$. We are left to conclude that $|P|=2$ or $|Q|=2$. But $(P, Q)$ is a cyclic 2-separation of $M \backslash c$, so either $P$ or $Q$ must be a parallel pair of $M \backslash c$; which implies the existence of a contradictory parallel pair in the 3 -connected matroid $M$. Thus $\operatorname{co}(M \backslash c)$ must be 3-connected.

Corollary 3.4.5. Let $M$ be a 3-connected matroid with a rank 3 cocircuit $C^{*}$ such that $\left|C^{*}\right| \geq 4$ and $r(M) \geq 4$. Let $B$ be a basis of $M$. Then there exists some $c \in C^{*} \cap(E(M)-B)$ such that $\operatorname{co}(M \backslash c)$ is 3-connected.

Proof. If $r\left(C^{*}-\{z\}\right)=3$ for all $z \in C^{*}$, the result follows immediately from Theorem 3.4.4 and the fact that $\left|C^{*}\right|>r\left(C^{*}\right)$. So assume that $r\left(C^{*}-\{d\}\right)=$ 2 for some $d \in C^{*}$. Now take any $c \in\left(C^{*}-\{d\}\right) \cap(E(M)-B) \neq \emptyset$. Then $r\left(C^{*}-\{c, d\}\right)=2$ because $M$ is 3 -connected and it follows that $r\left(C^{*}-\{c\}\right)=$ 3. Applying Theorem 3.4.4, we get that $\operatorname{co}(M \backslash c)$ is 3-connected.

Definition 3.4.6. Let $M$ be a 3 -connected matroid, and $B$ a basis of $M$. Suppose that for some $b_{1} \in B, \operatorname{si}\left(M / b_{1}\right)$ is not 3-connected. If $\left(X,\left\{b_{1}\right\}, Y\right)$ is a vertical 3-separation of $M$; where $Y=\left\{x_{1}, x_{2}, b_{2}, c\right\}$ is a cocircuit with $Y \cap B=\left\{b_{2}\right\}, \operatorname{si}\left(M / b_{2}\right)$ is not 3 -connected, and both $\left\{x_{1}, x_{2}, b_{2}\right\}$ and $\left\{b_{1}, c, b_{2}\right\}$ are triangles, then we shall call $Y$ a near-fan of $M$.

Corollary 3.4.7. Let $M$ be a 3-connected matroid and $B$ a basis of $M$. If $Y=\left\{x_{1}, x_{2}, b_{2}, c\right\} \subset E(M)$ is a near-fan of $M$, then both $\operatorname{co}\left(M \backslash x_{1}\right)$ and $\mathrm{co}\left(M \backslash x_{2}\right)$ are 3 -connected.

Proof. This is an immediate consequence of Theorem 3.4.4.

Theorem 3.4.8. Let $M$ be a 3-connected matroid with a triad $\{a, b, c\}$ and a circuit $\{a, b, c, d\}$. Then at least one of the following holds.

(i) Either $\operatorname{co}(M \backslash a)$ or $\operatorname{co}(M \backslash c)$ is 3-connected.

(ii) There exists $a^{\prime}, c^{\prime} \in E(M)$ such that both $\left\{a, a^{\prime}, b\right\}$ and $\left\{c, c^{\prime}, b\right\}$ are triangles. 
(iii) There exists $r \in E(M)$ such that $\{a, b, c, r\}$ is a coline.

Proof. We shall assume that neither (i) nor (ii) holds and prove that in this case, (iii) must hold. Now, as neither $\operatorname{co}(M \backslash a)$ nor $\operatorname{co}(M \backslash c)$ are 3-connected, it follows from Lemma 3.3.11 that there are cyclic 2-separations $(P, Q)$ and $(V, W)$ of $M \backslash a$ and $M \backslash c$ respectively.

Consider $(P, Q)$. We can assume without loss of generality that $d \in Q$. If $\{b, c\} \subseteq Q$, then $(P, Q \cup\{a\})$ is a 2-separation of $M$ as $a \in \operatorname{cl}(\{b, c, d\})$. So assume $b \in P$. Then $c \in \mathrm{cl}_{M \backslash a}^{*}(P)$ by Lemma 2.5.4, and it follows from Lemma 3.3.4 that $(P \cup\{c\}, Q-\{c\})$ is a cyclic 2-separation of $M \backslash a$. Thus, relabelling for clarity, we have a cyclic 2-separation $(X \cup\{b, c\}, Y)$ of $M \backslash a$ where $d \in Y$. Similarly, we obtain a cyclic 2-separation $(S \cup\{b, a\}, T)$ of $M \backslash c$ where $d \in T$.

3.4.8.1. If $\{b, c\}$ or $\{b, a\}$ is contained in a triangle, then the theorem holds.

Proof. Let $\left\{b, c, c^{\prime}\right\}$ be a triangle. By our original assumption, $\{b, a\}$ is not contained in a triangle. Consider the 2-separation $(S \cup\{b, a\}, T)$ of $M \backslash c$. As $S \cup\{a, b\}$ contains a circuit and $\{b, a\}$ is not in a triangle, we have $|S| \geq 2$. If $c^{\prime} \in S$, then $c \in \operatorname{cl}_{M}(S \cup\{b, a\})$, so that $(S \cup\{a, b, c\}, T)$ is a 2-separation of $M$. Thus $c^{\prime} \in T$. Now with the use of Lemma 2.6.5 we have

$$
\begin{aligned}
\lambda_{M \backslash c / b}(T) & =r_{M \backslash c / b}(T)+r_{M \backslash c / b}(S \cup\{a\})-r(M \backslash c / b) \\
& =r_{M \backslash c}(T \cup\{b\})-1+r_{M \backslash c}(S \cup\{a, b\})-1-(r(M \backslash c)-1) \\
& =r_{M \backslash c}(T)+r_{M \backslash c}(S \cup\{a, b\})-r(M) \\
& =r(M \backslash c)+1-r(M)=1
\end{aligned}
$$

Note that $r\left(\left\{a, b, c, c^{\prime}, d\right\}\right)=3$ because $\{a, b, c, d\}$ is a rank 3 circuit and $\left\{b, c, c^{\prime}\right\}$ is a triangle. So $r_{M \backslash c}\left(\left\{a, b, c^{\prime}, d\right\}\right)=3$. Therefore,

$$
r_{M \backslash c / b}\left(\left\{a, c^{\prime}, d\right\}\right)=r_{M \backslash c}\left(\left\{a, b, c^{\prime}, d\right\}\right)-1=2 .
$$

We also have $r_{M \backslash c / b}\left(\left\{c^{\prime}, d\right\}\right)=r_{M \backslash c}\left(\left\{b, c^{\prime}, d\right\}\right)-1=2$. Hence $a \in$ $\mathrm{cl}_{M \backslash c / b}\left(\left\{c^{\prime}, d\right\}\right) \subseteq \mathrm{cl}_{M \backslash c / b}(T)$. Therefore it must be that $\lambda_{M \backslash c / b}(T \cup\{a\})=1$. So $r_{M \backslash c / b}(T \cup\{a\})+r_{M \backslash c / b}(S)=r(M \backslash c / b)+1$ and

$$
\begin{aligned}
& r_{M \backslash c / b}(T \cup\{a\})+r_{M \backslash c / b}(S)=r(M \backslash c / b)+1 \\
\Longrightarrow & r_{M \backslash c}(T \cup\{a, b\})+r_{M \backslash c / b}(S)=r(M \backslash c / b)+2
\end{aligned}
$$




$$
\begin{gathered}
\Longrightarrow r_{M \backslash c}(T \cup\{a, b\})+r_{M \backslash c / b}(S)=r(M)+1 \\
\Longrightarrow r_{M \backslash c}(T \cup\{a, b\})+r_{M \backslash c}(S)=r(M)+1
\end{gathered}
$$

So that $\lambda_{M \backslash c}(T \cup\{a, b\})=1$. But then $c \in \operatorname{cl}(\{a, b, d\})$ implies that $\lambda_{M}(T \cup$ $\{a, b, c\})=1$, contradicting the fact that $M$ is 3-connected. Therefore $\{b, c\}$ can not be contained in a triangle. Similarly for $\{b, a\}$.

Thus we may now assume that neither $\{b, a\}$ nor $\{b, c\}$ is contained in a triangle. From this and the fact that both $X \cup\{b, c\}$ and $S \cup\{b, a\}$ contain circuits, we deduce that $|X| \geq 2$ and $|S| \geq 2$. In what follows, let $M^{\prime}=$ $M \backslash\{a, b, c\}$.

3.4.8.2. $c \in \operatorname{cl}(X \cup\{b\})$ and $a \in \operatorname{cl}(S \cup\{b\})$.

Proof. Suppose that $c \notin \operatorname{cl}(X \cup\{b\})$. Then $r(X)=r(X \cup\{b, c\})-2$. But $r\left(M^{\prime}\right)=r(M)-1$. We have

$$
\begin{gathered}
r(X \cup\{b, c\})+r(Y) \leq r(M)+1 \\
\Longrightarrow r(X)+2+r(Y) \leq r\left(M^{\prime}\right)+2 \\
\Longrightarrow r(X)+r(Y) \leq r\left(M^{\prime}\right)
\end{gathered}
$$

So that $(X, Y)$ is a separation of $M^{\prime}$. But $r(Y \cup\{b, c\}) \leq r(Y)+2$, and $a \in \mathrm{cl}_{M}(Y \cup\{b, c\})$, so that $r(Y \cup\{a, b, c\}) \leq r(Y)+2$. Now

$$
\begin{aligned}
r(X)+r(Y \cup\{a, b, c\}) & \leq r(X)+r(Y)+2 \\
& \leq r\left(M^{\prime}\right)+2 \\
& \leq r(M)+1 .
\end{aligned}
$$

This gives $(X, Y \cup\{a, b, c\})$ as a 2-separating partition of $M$; which is contradictory as $|X| \geq 2$. Similarly $a \in \operatorname{cl}(S \cup\{b\})$.

3.4.8.3. $\lambda_{M^{\prime}}(X)=\lambda_{M^{\prime}}(Y)=\lambda_{M^{\prime}}(S)=\lambda_{M^{\prime}}(T)=1$. 
Proof. Using 3.4.8.2,

$$
\begin{aligned}
\lambda_{M^{\prime}}(X) & =r_{M^{\prime}}(X)+r_{M^{\prime}}(Y)-r\left(M^{\prime}\right) \\
& =r_{M^{\prime}}(X)+r_{M^{\prime}}(Y)-r(M)+1 \\
& =r_{M \backslash a}(X)+r_{M \backslash a}(Y)-r(M)+1 \\
& =r_{M \backslash a}(X \cup\{b\})-1+r_{M \backslash a}(Y)-r(M)+1 \\
& =r_{M \backslash a}(X \cup\{b, c\})+r_{M \backslash a}(Y)-r(M) \\
& =r(M)+1-r(M) \\
& =1=\lambda_{M^{\prime}}(Y)
\end{aligned}
$$

The result that $\lambda_{M^{\prime}}(S)=\lambda_{M^{\prime}}(T)=1$ follows similarly.

3.4.8.4. If $Z \subseteq X$ or $Z \subseteq S$, then $\lambda_{M}(Z) \leq \lambda_{M^{\prime}}(Z)+1$.

Proof. Suppose $Z \subseteq X$. Let $Z^{\prime}=E\left(M^{\prime}\right)-Z$. Note that $d \in Z^{\prime}$ so $r\left(Z^{\prime} \cup\right.$ $\{a, b, c\}) \leq r\left(Z^{\prime}\right)+2$. Then $r(M)=r\left(M^{\prime}\right)+1$ implies that

$$
\begin{aligned}
\lambda_{M}(Z) & =r(Z)+r\left(Z^{\prime} \cup\{a, b, c\}\right)-r(M) \\
& =r_{M^{\prime}}(Z)+r\left(Z^{\prime} \cup\{a, b, c\}\right)-r\left(M^{\prime}\right)-1 \\
& \leq r_{M^{\prime}}(Z)+r\left(Z^{\prime}\right)+2-r\left(M^{\prime}\right)-1 \\
& \leq r_{M^{\prime}}(Z)+r_{M^{\prime}}\left(Z^{\prime}\right)-r\left(M^{\prime}\right)+1 \\
& \leq \lambda_{M^{\prime}}(Z)+1
\end{aligned}
$$

Similarly if $Z \subseteq S$.

3.4.8.5. If $Z \subseteq Y-\{d\}$ or $Z \subseteq T-\{d\}$, then $\lambda_{M}(Z)=\lambda_{M^{\prime}}(Z)$.

Proof. Suppose $Z \subseteq Y-\{d\}$. Let $Z^{\prime}=E\left(M^{\prime}\right)-Z$. Note that $d \in Z^{\prime}$ and $X \subset Z^{\prime}$. Now $r\left(Z^{\prime} \cup\{b\}\right)=r\left(Z^{\prime}\right)+1$, but by 3.4.8.2, $c \in \operatorname{cl}(X \cup\{b\})$, so $c \in \operatorname{cl}\left(Z^{\prime} \cup\{b\}\right)$. Hence, $r\left(Z^{\prime} \cup\{b, c\}\right)=r\left(Z^{\prime}\right)+1$. But then $a \in \operatorname{cl}\left(Z^{\prime} \cup\{b, c\}\right)$, so $r\left(Z^{\prime} \cup\{a, b, c\}\right)=r\left(Z^{\prime}\right)+1$. Therefore

$$
\begin{aligned}
\lambda_{M}(Z) & =r(Z)+r\left(Z^{\prime} \cup\{a, b, c\}\right)-r(M) \\
& =r(Z)+r\left(Z^{\prime}\right)+1-r(M) \\
& =r(Z)+r\left(Z^{\prime}\right)-r\left(M^{\prime}\right)=\lambda_{M^{\prime}}(Z)
\end{aligned}
$$

as required. Similarly for any $Z \subseteq T-\{d\}$. 
3.4.8.6. $T \cap X \neq \emptyset$, and $S \cap Y \neq \emptyset$.

Proof. If $T \cap X=\emptyset$, then $X \subseteq S$, and by 3.4.8.2, $\{a, c\} \subseteq \operatorname{cl}(S \cup\{b\})$. We once again obtain a contradictory 2-separation $(S \cup\{a, b, c\}, T)$ of $M$. Similarly for $S \cap Y$.

Applying submodularity of the connectivity function together with 3.4.8.3 we have

$$
\begin{gathered}
\lambda_{M^{\prime}}(T \cup X)+\lambda_{M^{\prime}}(T \cap X) \leq \lambda_{M^{\prime}}(T)+\lambda_{M^{\prime}}(X) \\
\Longrightarrow \lambda_{M^{\prime}}(S \cap Y)+\lambda_{M^{\prime}}(T \cap X) \leq 1+1=2
\end{gathered}
$$

But by 3.4.8.5, $\lambda_{M^{\prime}}(S \cap Y)=\lambda_{M}(S \cap Y)$ and $\lambda_{M^{\prime}}(T \cap X)=\lambda_{M}(T \cap X)$. This result combined with 3.4.8.6 and the fact that $M$ is 3-connected implies that we must have $|S \cap Y|=|T \cap X|=1$. We also know that $|Y| \geq 3$ because $(X \cup\{b, c\}, Y)$ is cyclic. Thus $|Y \cap T| \geq 2$.

3.4.8.7. $\lambda_{M^{\prime}}(Y \cap T) \geq 2$.

Proof. By 3.4.8.2, $\{a, c\} \subseteq \operatorname{cl}(X \cup S \cup\{b\})$, so as $r(X \cup S \cup\{b\})=r(X \cup S)+1$, we have $r(X \cup S \cup\{a, b, c\})=r(X \cup S)+1$. Hence $\lambda_{M}(Y \cap T, X \cup S \cup\{a, b, c\})=$ $\lambda_{M^{\prime}}(Y \cap T, X \cup S)$. Thus, if $\lambda_{M^{\prime}}(Y \cap T) \in\{0,1\}$, we contradict the 3connectivity of $M$.

Now $\lambda_{M^{\prime}}(S \cap X)+\lambda_{M^{\prime}}(T \cap Y) \leq \lambda_{M^{\prime}}(S)+\lambda_{M^{\prime}}(X) \leq 1+1=2$. So by 3.4.8.7, $\lambda_{M^{\prime}}(S \cap X)=0$. By 3.4.8.4, we deduce that $|S \cap X|=1$. Let $S \cap X=\{r\}$. Let $\{a, b, c\}=A$. As $\lambda_{M^{\prime}}(S \cap X)=0$, we have $\lambda_{M^{\prime}}(Y \cup T)=$ 0 . Therefore $r_{M}(Y \cup T)=r_{M^{\prime}}(Y \cup T)=r\left(M^{\prime}\right)-1=r(M)-2$. Thus $\lambda_{M}(Y \cup T)=r(M)-2+r(A \cup\{r\})-r(M)=r(A \cup\{r\})-2 \leq 2$, so that $(Y \cup T, A \cup\{r\})$ must be a 3-separation of $M$. Now by Lemma 3.2.7, $r \in \mathrm{cl}^{(*)}(A)$. If $r \in \mathrm{cl}(A)$, then $(S \cup\{a, b, c\}, T)$ is a contradictory 2-separation of $M$. We conclude that $r \in \mathrm{cl}^{*}(A)$ and that $\{a, b, c, r\}$ is a coline, giving (iii). 


\subsection{Fixed Basis Connectivity}

Given a 3-connected matroid $M$ with $e \in E(M)$, Bixby's Lemma (see 3.4.2) tells us that either $\operatorname{si}(M / e)$ or $\operatorname{co}(M \backslash e)$ is 3-connected. However, situations can arise where we specifically want to contract certain elements, or delete certain elements, all while maintaining 3-connectivity. In such circumstances, Bixby's Lemma may be of limited use. This section introduces one important example of such a situation.

Suppose we have an $\mathbb{F}$-representable matroid $M$. As $M$ is invariant under performing standard row operations on a representation of $M$, it is natural that we want to deal with representations of the form $\left[I_{r}, D\right]$ where $I_{r}$ is an $r \times r$ identity matrix and $D$ is an $r \times(|E(M)|-r)$ matrix. Such a matrix representation is said to be in standard form. Now if $A=\left[I_{r}, D\right]$ is a representation of $M$ over $\mathbb{F}$ in standard form, then the set of column vectors contained in $I_{r}$ form a basis for the space $\mathbb{F}^{r}$. As the rank of a matroid corresponds to the dimension of the rowspace in a representation of that matroid, it then follows that $r(M)=r$, and therefore, the set of column labels for columns in $I_{r}$ constitute a basis $B$ of the matroid $M$. Hence from our natural desire to work with representations that are in standard form, we are in effect choosing a particular basis for $M$. Any information present in the submatrix $D$ is observed relative to the basis $B$. Clearly, a matroid will have many possible standard form representations. For example, if $d$ is in some basis $B_{d}$ of $M$, and $d$ corresponds to some column of $D$, then (see Lemma 2.1.7), for some $i \in\{1, \ldots, r\}$, we may pivot on the i-th entry of $d-$ transforming it into the i-th unit vector - and interchange columns $d$ and $i$. The resulting matrix is another standard form representation $A^{\prime}=\left[I_{r}, G\right]$ of $M$; corresponding to the basis $B^{\prime}=(B-\{i\}) \cup\{d\}$ of $M$. However in general, the entries in the submatrix $G$ will differ significantly from the entries of the submatrix $D$. Thus, important information that may be present in $D$ can be lost when switching to the representation $A^{\prime}$ relative to the new basis $B^{\prime}$.

We observed at the end of Section 2.6 that an $\mathbb{F}$-representation for any minor $N$ of an $\mathbb{F}$-representable matroid $M$ can be found by taking an $\mathbb{F}$ representation $A$ of $M$ and performing a sequence of operations consisting of pivots, row deletions and column deletions. We will examine what happens when our starting representation $A$ of $M$ is in standard form. Suppose that $\left[I_{r}, D\right]$ is a representation - in standard form - of $M$. Then the columns of $I_{r}$ correspond to a basis $B$ of $M$, and therefore, the columns of $D$ correspond 
to a cobasis $E(M)-B$ of $M$.

Suppose that $d \in E(M)-B$. Then $d$ corresponds to some column of the submatrix $D$. We will assume that $d$ is not a loop of $M$ to avoid the trivial case. Consider the matroid $M / d$. Recall that the first step in obtaining a representation of $M / d$ is to pivot on some non-zero i-th entry of column $d$; transforming it into the i-th unit vector. If we then switch columns $d$ and $i$ before completing our contraction of $d$, we find that - upon contracting $d$ - we obtain a representation of $M / d$ which is in standard form. But, as observed above, such a procedure corresponds to changing the basis with which we are observing the representation with respect to, and carrying out such a process means changing potentially important information present in the submatrix $D$. Now consider the matroid $M \backslash d$. To obtain a representation for $M \backslash d$, we simply delete column $d$ of $D$. Doing so gives a representation — in standard form — of $M \backslash d$. Moreover, in this case, all remaining entries in the submatrix $D$ are completely unchanged.

Now suppose that $b \in B$. Then $b$ corresponds to some column of the identity matrix $I_{r}$. Consider the matroid $M \backslash b$. We obtain a representation of this matroid by deleting column $b$, but doing so leaves us with a representation which is not in standard form. To then find a standard form representation of $M \backslash b$, we need to pivot on a non-zero entry of some column of $D$ to obtain another unit vector. But doing so will again often perturb information present in entries of $D$ which we may be wanting to maintain. On the other hand, consider the matroid $M / b$. As $b$ already represents some i-th unit vector, contracting $b$ simply corresponds to removing row $i$ and column $b$. This gives us a representation of $M / b$ which is in standard form, where all entries of $D$ that remain are unaltered.

So suppose we have a representation $\left[I_{r}, D\right]$ — in standard form - of a matroid $M$ over $\mathbb{F}$. As discussed, this corresponds to a particular basis $B$ and cobasis $E(M)-B$ of $M$. Now suppose that one wished to study a representation $\left[I_{q}, D^{\prime}\right]$ of a minor $N$ of $M$ over $\mathbb{F}$, and one required that relevant information present in $D$ remain observable in $D^{\prime}$. As we've seen, such a requirement can be met as long as we restrict ourselves to the situation where $N$ is some minor obtained from $M$ by contracting elements from the basis $B$ and/or deleting elements of the cobasis $E(M)-B$. If $N$ is a minor of $M$ found in such a manner, then a representation $\left[I_{q}, D^{\prime}\right]$ - in standard form - of $N$ is easily found, and entries of $D^{\prime}$ correspond identically to entries present in the submatrix $D$ of our original representation. 
For the remainder of this thesis, we are interested in what can be done in such a situation. From a matroidal point of view, we have some matroid $M$, and some particular basis $B$ of $M$, and we want to study minors of $M$ that are found by contracting elements of $B$ and deleting elements of $E(M)-B$. But of course, even for minors found in this way, if the representations themselves are to be of any use as an inductive tool, we need some sense of uniqueness of representations; and for that, we require 3-connectivity.

Oxley et al [10] studied this problem and found that: given a matroid $M$ and basis $B$ of $M$; provided $M$ has no 4-element fans, there is always either an element $b \in B$ such $M / b$ is 3-connected, or an element $c \in E(M)-B$ such that $M \backslash c$ is 3 -connected. In this work, we are predominantly concerned with what can be done when wanting to maintain 3-connectivity up to parallel and series classes. We make the following definition:

Definition 3.5.1. Let $M$ be a 3-connected matroid, $B$ a basis of $M$, and $e \in E(M)$. We say that $e$ is removable with respect to $B$ if either:

(i) $e \in B$ and $\operatorname{si}(M / e)$ is 3-connected, or

(ii) $e \notin B$ and $\operatorname{co}(M \backslash e)$ is 3-connected.

The following two lemmas show that the property of being removable with respect to a basis behaves nicely under duality.

Lemma 3.5.2. Let $M$ be a 3-connected matroid, and let $B$ be a basis of $M$. Then $e \in E(M)$ is removable with respect to $B$ if and only if e is removable with respect to the basis $E(M)-B$ in $M^{*}$.

Proof. $e \in E(M)$ is removable with respect to $B$ iff $e \in B$ and $\operatorname{si}(M / e)$ is 3-connected, or $e \notin B$ and $\operatorname{co}(M \backslash e)$ is 3-connected; iff $e \notin E(M)-B$ and $(\mathrm{si}(M / e))^{*}$ is 3-connected, or $e \in E(M)-B$ and $(\operatorname{co}(M \backslash e))^{*}$ is 3-connected; iff $e \notin E(M)-B$ and $\operatorname{co}\left(M^{*} \backslash e\right)$ is 3 -connected, or $e \in E(M)-B$ and $\operatorname{si}\left(M^{*} / e\right)$ is 3 -connected; iff $e$ is removable with respect to the basis $E(M)-B$ in $M^{*}$.

Lemma 3.5.3. Let $M$ be a 3-connected matroid, and let $B$ be a basis of $M$. Let $K$ denote the set of elements which are removable with respect to $B$. Let $K^{*}$ denote the set of elements which are removable with respect to the basis $E(M)-B$ in $M^{*}$. Then $K=K^{*}$. 
Proof. This follows immediately from Lemma 3.5.2.

In proving results on 3-connectivity in matroid theory, it is often advantageous to exclude certain substructures from the matroids we are considering. Fans are an example of such a substructure.

Definition 3.5.4. Let $M$ be a matroid. A set $F \subseteq E(M)$ is a fan if there is an ordering $\left(f_{1}, \ldots, f_{n}\right)$ of the elements of $F$ such that, for all $i \in\{1, \ldots, n-2\}$,

(i) $\left\{f_{i}, f_{i+1}, f_{i+2}\right\}$ is either a triangle or a triad, and

(ii) when $\left\{f_{i}, f_{i+1}, f_{i+2}\right\}$ is a triangle, $\left\{f_{i+1}, f_{i+2}, f_{i+3}\right\}$ is a triad, and when $\left\{f_{i}, f_{i+1}, f_{i+2}\right\}$ is a triad, $\left\{f_{i+1}, f_{i+2}, f_{i+3}\right\}$ is a triangle.

The focus of the work following will be on matroids that do not contain any 4-element fans, that is: fans of cardinality four. Excluding matroids that do contain such structures is not greatly confining for our purposes. For example, it can readily be shown that any excluded minor for $G F(q)$ does not contain any 4-element fans. The following lemma notes that fans are also well behaved under duality.

Lemma 3.5.5. Let $M$ be a matroid. Then $F$ is a fan of $M$ if and only if it is a fan of $M^{*}$. In particular, if $M$ does not contain any 4-element fans, then neither does $M^{*}$.

Proof. This follows directly from the definition of a fan and the fact that triangles and triads are dual to one another.

Although not explicitly stated therein, it can be deduced directly from results in the paper by Oxley et al [10] that for a matroid $M$ with no 4element fans, and a basis $B$ of $M$, there always exists two distinct elements which are removable with respect to $B$. In the next chapter, we look to strengthen this result. 


\section{Chapter 4}

\section{The Existence of Removable Elements}

In this chapter we prove the following result:

Theorem 4.0.1. Let $M$ be a 3-connected matroid with no 4-element fans. Let $B$ be a basis of $M$. Let $K$ denote the set of elements which are removable with respect to $B$. If $|E(M)| \geq 4$, then $|K| \geq 4$.

\subsection{Preliminary Results}

Theorem 4.1.1. Let $M$ be a 3-connected matroid with no 4-element fans. Let $B$ be a basis of $M$ such that for some $b \in B, \operatorname{si}(M / b)$ is not 3-connected. Let $(X,\{b\}, Y)$ be a vertical 3-separation of $M$ with $Y \cup\{b\}$ closed. Then either:

(i) there exists $b_{1}, b_{2} \in X \cap B$ with $b_{1} \neq b_{2}$ such that both $\operatorname{si}\left(M / b_{1}\right)$ and $\operatorname{si}\left(M / b_{2}\right)$ are 3 -connected, or

(ii) there exists $b_{1} \in X \cap B, d_{1} \in X \cap(E(M)-B)$ such that both $\operatorname{si}\left(M / b_{1}\right)$ and $\operatorname{co}\left(M \backslash d_{1}\right)$ are 3-connected, or

(iii) there exists $d_{1}, d_{2} \in X \cap(E(M)-B)$ with $d_{1} \neq d_{2}$ such that both $\operatorname{co}\left(M \backslash d_{1}\right)$ and $\operatorname{co}\left(M \backslash d_{2}\right)$ are 3 -connected, or 
(iv) $X \cap B=\left\{b_{c}\right\}, \operatorname{si}\left(M / b_{c}\right)$ is 3-connected, $X=\left\{a, b_{c}, c\right\}, X \cup\{b\}$ is a circuit, and neither $\operatorname{co}(M \backslash a)$ nor $\operatorname{co}(M \backslash c)$ is 3-connected.

Proof. We shall assume that (i) does not hold and show that either (ii), (iii) or (iv) does. The proof is separated into 2 cases according the possible structure of $X$.

Case (i): $X$ contains no element $b_{x}$ such that $\operatorname{si}\left(M / b_{x}\right)$ is 3 -connected.

Assume as stated that for all $b_{x} \in B \cap X, \operatorname{si}\left(M / b_{x}\right)$ is not 3-connected. We shall show that in this case, (iii) must hold.

As $b \in \operatorname{cl}(X)$ and $r(X) \geq 3$, we have $r(Y)<r(M)$, so that $X \cap B \neq$ $\emptyset$. Note that for each $b_{x} \in B \cap X$, there exists a vertical 3-separation $\left(S_{b_{x}},\left\{b_{x}\right\}, T_{b_{x}}\right)$ of $M$. If, for some $b_{x} \in X \cap B$, there is a vertical 3separation $\left(S,\left\{b_{x}\right\}, T\right)$ of $M$ such that $S$ or $T$ is contained in $X \cup\{b\}$, then by Lemma 3.3.6 and a possible relabelling, there exists such a vertical 3-separation where $S \subseteq X \cup\{b\}$ and $T \cup\left\{b_{x}\right\}$ is closed. Then $S \subseteq\left(X-\left\{b_{x}\right\}\right) \cup\{b\}$. If equality holds here, then $T=Y$. But $b_{x} \in \operatorname{cl}(T)$ so $b_{x} \in \operatorname{cl}(Y)$; a contradiction. Hence $S \subset\left(X-\left\{b_{x}\right\}\right) \cup\{b\}$. Now relabel so that $\left(S,\left\{b_{x}\right\}, T\right)$ becomes $(X,\{b\}, Y)$. By an iteration of this procedure, we eventually obtain a vertical 3-separation $(X,\{b\}, Y)$ of $M$ with $Y \cup\{b\}$ closed such that if $\left(S,\left\{b_{x}\right\}, T\right)$ is a vertical 3-separation of $M$ with $b_{x} \in X \cap B$, then neither $S$ nor $T$ is contained in $X \cup\{b\}$. Moreover, we maintain the property that there is no element $b_{x} \in X \cap B$ such that $\operatorname{si}\left(M / b_{x}\right)$ is 3-connected.

Let $b_{x}$ be an element of $X \cap B$, and let $\left(P,\left\{b_{x}\right\}, Q\right)$ be a vertical 3separation of $M$. Without loss of generality, we may assume that $b \in Q$. Moreover, we may assume by Lemma 3.3.6 that $Q \cup\left\{b_{x}\right\}$ is closed.

4.1.1.1. Each of the intersections $X \cap P, X \cap Q, Y \cap P$, and $Y \cap Q$ is nonempty.

Proof. If $X \cap P$ or $X \cap Q$ is empty, then $P$ or $Q$ is contained in $Y \cup\{b\}$, and so $b_{x} \in \operatorname{cl}(Y \cup\{b\})$; which contradicts the fact that $Y \cup\{b\}$ is closed. Thus $X \cap P$ and $X \cap Q$ are non-empty. If $Y \cap P$ or $Y \cap Q$ is empty, then $P$ or $Q$ is contained in $X \cup\{b\}$; a contradiction.

4.1.1.2. $\lambda(X \cap P) \leq 2$. 
Proof. As $E-(X \cup P)=(Y \cap Q) \cup\{b\}$, we have $|E-(X \cup P)| \geq 2$. Thus, since $\lambda(X)=2=\lambda(P)$, it follows by uncrossing that $\lambda(X \cap P) \leq 2$.

4.1.1.3. $r\left((X \cap P) \cup\left\{b_{x}\right\}\right)=2$

Proof. If $|X \cap P|=1$, this follows immediately as $M$ is 3 -connected. Since $X \cap P$ is non-empty, we may now assume that $|X \cap P| \geq 2$. We have $\lambda(X)=2=\lambda\left(P \cup\left\{b_{x}\right\}\right)$, and $\left|E-\left(X \cup\left(P \cup\left\{b_{x}\right\}\right)\right)\right| \geq 2$, so that, by uncrossing, $\lambda\left(X \cap\left(P \cup\left\{b_{x}\right\}\right)\right) \leq 2$. With 4.1.1.2, we deduce that $\lambda(X \cap$ $P)=\lambda\left((X \cap P) \cup\left\{b_{x}\right\}\right)=2$. By Lemma 3.2.7, $b_{x} \in \mathrm{cl}^{(*)}(X \cap P)$. If $b_{x} \in \mathrm{cl}^{*}(X \cap P)$, then by Lemma 2.5.4, $b_{x} \notin \mathrm{cl}(Y \cup Q)$; a contradiction. So $b_{x} \in \operatorname{cl}(X \cap P)$. If $r\left((X \cap P) \cup\left\{b_{x}\right\}\right) \geq 3$, then $\left(X \cap P,\left\{b_{x}\right\}, Y \cup Q\right)$ is a vertical 3 -separation of $M$. But this contradicts our construction of $(X,\{b\}, Y)$. So $r\left((X \cap P) \cup\left\{b_{x}\right\}\right)=2$.

Suppose $|Y \cap P|=1$. Then $|X \cap P| \geq 2$. If $|X \cap P|=2$, then, as $Q \cup\left\{b_{x}\right\}$ is closed, $P$ is a triad. As $(X \cap P) \cup\left\{b_{x}\right\}$ is a triangle, $P \cup\left\{b_{x}\right\}$ is a 4-element fan: a contradiction. Thus we may assume that $|X \cap P| \geq 3$. Then $(X \cap P) \cup\left\{b_{x}\right\}$ is a rank-2 set having at least four elements. By Lemmas 3.2.2 and 2.3.3, we obtain distinct $d_{1}, d_{2} \in(E-B) \cap X$ such that both $M \backslash d_{1}$ and $M \backslash d_{2}$ are 3-connected, so (iii) holds. So assume that $|Y \cap P| \geq 2$.

4.1.1.4. $r\left((X \cap Q) \cup\left\{b, b_{x}\right\}\right)=2$.

Proof. Since $\lambda(X \cup\{b\})=2=\lambda\left(Q \cup\left\{b_{x}\right\}\right)$ and $\left|E-\left((X \cup\{b\}) \cup\left(Q \cup\left\{b_{x}\right\}\right)\right)\right|=$ $|Y \cap P| \geq 2$, it follows by uncrossing that $\lambda\left((X \cap Q) \cup\left\{b, b_{x}\right\}\right) \leq 2$. But $\left|(X \cap Q) \cup\left\{b, b_{x}\right\}\right| \geq 3$ and so $\lambda\left((X \cap Q) \cup\left\{b, b_{x}\right\}\right)=2$. Noting that $P \subseteq E-\left((X \cap Q) \cup\left\{b, b_{x}\right\}\right)$, we have $b_{x} \in \operatorname{cl}\left(E-\left((X \cap Q) \cup\left\{b, b_{x}\right\}\right)\right)$, and it follows from Lemmas 3.2.7 and 3.2.8 that $b_{x} \in \operatorname{cl}((X \cup\{b\}) \cap Q)$. If $r\left((X \cap Q) \cup\left\{b, b_{x}\right\}\right) \geq 3$, then it follows that $\left((X \cup\{b\}) \cap Q,\left\{b_{x}\right\}, E-\right.$ $\left.\left((X \cap Q) \cup\left\{b, b_{x}\right\}\right)\right)$ is a vertical 3-separation that contradicts the choice of $b$. Therefore, $r\left((X \cap Q) \cup\left\{b, b_{x}\right\}\right) \leq 2$ and 4.1.1.4 holds.

Now let $L_{1}=\{b\} \cup(X \cap Q)$ and $L_{2}=\left\{b_{x}\right\} \cup(X \cap P)$. We have $\left|L_{1}\right| \geq 2$ and $\left|L_{2}\right| \geq 2$, and both $\operatorname{cl}\left(L_{1}\right)$ and $\operatorname{cl}\left(L_{2}\right)$ are lines. By 4.1.1.1, $\left|\operatorname{cl}\left(L_{1}\right)\right| \geq 3$. If $\left|\operatorname{cl}\left(L_{1}\right)\right| \geq 4$, then by Lemmas 3.2 .2 and 2.3 .3 we again obtain distinct $d_{1}, d_{2} \in(E-B) \cap X$ such that (iii) holds. So we may assume that $\left|\operatorname{cl}\left(L_{1}\right)\right|=3$. Let $\operatorname{cl}\left(L_{1}\right)-\left\{b, b_{x}\right\}=\{a\}$. Suppose that $\left(L_{2}-\left\{b_{x}\right\}\right) \cap B=\left\{b^{\prime}\right\}$. Then, in $M / b^{\prime}$, each of $b, b_{x}$, and $a$ are contained in some distinct parallel class by an 
application of Lemma 2.6.5. Thus, by Lemma 3.4.1, it follows that $\operatorname{si}\left(M / b^{\prime}\right)$ is 3-connected: a contradiction. We conclude that $\left(L_{2}-\left\{b_{x}\right\}\right) \cap B=\emptyset$. If $\left|\operatorname{cl}\left(L_{2}\right)\right| \geq 4$, then $\left|L_{2} \cap(E-B)\right| \geq 2$ by Lemma 2.3.3, so by Lemma 3.2.2, (iii) holds. Thus we may assume that $\left|\operatorname{cl}\left(L_{2}\right)\right| \in\{2,3\}$. Since $M$ has no 4-element fans, and $Y \cup\{b\}$ is closed it must be that $\left|L_{2}-\left\{b_{x}\right\}\right|=2$. Let $L_{2}-\left\{b_{x}\right\}=\left\{x_{1}, x_{2}\right\}$. Note that $\left\{a, b_{x}, x_{1}, x_{2}\right\}$ is a cocircuit of $M$ and $X$ is a near-fan of $M$. Applying Corollary 3.4.7, we have that $\operatorname{co}\left(M \backslash x_{1}\right)$ and $\operatorname{co}\left(M \backslash x_{2}\right)$ are 3 -connected so that (iii) holds.

Case (ii): $X$ contains exactly one $b_{c} \in B$ such that $\operatorname{si}\left(M / b_{c}\right)$ is 3 -connected.

Now assume as stated that there is exactly one $b_{c} \in B \cap X$ such that $\operatorname{si}\left(M / b_{c}\right)$ is 3-connected. If, for some $b_{x} \in(X \cap B)-\left\{b_{c}\right\}$, there is a vertical 3-separation $\left(S,\left\{b_{x}\right\}, T\right)$ of $M$ such that $S$ or $T$ is contained in $(X \cup\{b\})-$ $\left\{b_{c}\right\}$, then there is such a vertical 3-separation such that $S \subseteq(X \cup\{b\})-$ $\left\{b_{c}\right\}$ and $T \cup\left\{b_{x}\right\}$ is closed. We may now apply case (i) to the vertical 3separation $\left(S,\left\{b_{x}\right\}, T\right)$ to conclude that (iii) holds in addition to $\operatorname{si}\left(M / b_{c}\right)$ being 3-connected.

So we may assume that if $\left(P,\left\{b_{x}\right\}, Q\right)$ is a vertical 3-separation of $M$ with $b_{x} \in(X \cap B)-\left\{b_{c}\right\}$, then neither $P$ nor $Q$ is contained in $(X \cup\{b\})-$ $\left\{b_{c}\right\}$. Furthermore, by Lemma 3.3.6, we may assume that $Q \cup\left\{b_{x}\right\}$ is closed. Supposing that $(X \cap B)-\left\{b_{c}\right\} \neq \emptyset$, let $b_{x} \in(X \cap B)-\left\{b_{c}\right\}$, and let $\left(P,\left\{b_{x}\right\}, Q\right)$ be a vertical 3-separation with $Q \cup\left\{b_{x}\right\}$ closed. Without loss of generality, we may assume that $b \in Q$. Observe that 4.1.1.1, 4.1.1.2, 4.1.1.3, and 4.1.1.4 all hold. We deduce that $b_{c} \in X \cap P$. Letting $L_{1}=\{b\} \cup(X \cap Q)$ and $L_{2}=\left\{b_{x}\right\} \cup(X \cap P)$ as per the argument following 4.1.1.4, we again can assume that $\left|\operatorname{cl}\left(L_{1}\right)\right|=\left|\operatorname{cl}\left(L_{2}\right)\right|=3$. Letting $L_{2}-\left\{b_{c}, b_{x}\right\}=\left\{x_{1}\right\}$ it follows by an application of Theorem 3.4.4 that $\operatorname{co}\left(M \backslash x_{1}\right)$ is 3-connected. Thus (ii) is satisfied.

The final possibility to consider is when $(X \cap B)-\left\{b_{c}\right\}=\emptyset$. Here $r(Y \cup\{b\})=r(M)-1$ so that $X$ is a cocircuit with $r(X)=3$. If $|X| \geq 4$, then Corollary 3.4.5 gives us (ii). So assume $|X|=3$. Then $X$ is a triad, and as $M$ contains no 4-element fans, $X \cup\{b\}$ must be a circuit. Let $X-\left\{b_{c}\right\}=\{a, c\}$. If $\operatorname{co}(M \backslash a)$ or $\operatorname{co}(M \backslash c)$ is 3-connected, then (ii) holds. Otherwise, we have (iv). We conclude that the theorem holds.

The next result is an extension of Theorem 4.1.1 restated in a form which will be useful in what follows. 
Theorem 4.1.2. Let $M$ be a 3-connected matroid with no 4-element fans. Let $B$ be a basis of $M$ such that for some $b \in B$, si $(M / b)$ is not 3-connected. Let $(X,\{b\}, Y)$ be a vertical 3-separation of $M$ with $Y \cup\{b\}$ closed. Let $K$ denote the set of elements of $M$ removable with respect to $B$. Then either:

(i) $|X \cap K| \geq 2$, or

(ii) $|X \cap K|=1, X$ is a triad, there exists $r \in Y \cap K$ such that $X \cup\{r\}$ is a 4-point coline with $|(X \cup\{r\}) \cap B|=2$, and $(Y \cup\{b\})-\{r\}$ is closed.

Proof. Assume that (i) does not hold. Applying Theorem 4.1.1, it must be that 4.1.1 (iv) holds. So $X$ is a triad, $X \cup\{b\}$ is a circuit, and $X \cap K=X \cap B$. Now apply Theorem 3.4.8. As $|X \cap K|=1,3.4 .8$ (i) doesn't hold. If 3.4.8 (ii) holds, then $X \cup\left\{a^{\prime}\right\}$ and $X \cup\left\{c^{\prime}\right\}$ are 4-element fans of $M$; which cannot be. So 3.4.8 (iii) must hold. Let $X \cup\{r\}$ be the given 4-point coline of $M$. It follows by Lemmas 3.2.3 and 2.5.6 that $|(X \cup\{r\}) \cap B|=2$. Finally, as $z \in \operatorname{cl}^{*}((X \cup\{r\})-\{z\})$ for all $z \in X \cup\{r\}$, Lemma 2.5.4 implies that $(Y \cup\{b\})-\{r\}$ is closed.

Corollary 4.1.3. Let $M$ be a 3-connected matroid with no 4-element fans. Let $B$ be a basis of $M$ such that for some $c \in E(M)-B, \operatorname{co}(M \backslash c)$ is not 3connected. Let $(S,\{c\}, T)$ be a cyclic 3 -separation of $M$ with $T \cup\{c\}$ coclosed. Let $K$ denote the set of elements of $M$ which are removable with respect to $B$. Then either:

(i) $|S \cap K| \geq 2$, or

(ii) $|S \cap K|=1, S$ is a triangle, there exists $r \in T \cap K$ such that $S \cup\{r\}$ is a 4-point line with $|(T \cup\{r\}) \cap(E(M)-B)|=2$, and $(T \cup\{c\})-\{r\}$ is coclosed.

Proof. This follows by dualising Theorem 4.1.2 and using Lemmas 3.3.3, 3.5.3, and 3.5.5. 


\subsection{The Minimal Number of Removable Elements}

We now extend the results from the previous section to place a lower bound on the number of removable elements in a suitable matroid as a whole. We conclude this chapter with a proof of Theorem 4.0.1.

Theorem 4.2.1. Let $M$ be a 3-connected matroid with no 4-element fans. Let $B$ be a basis of $M$ such that for some $b \in B, \operatorname{si}(M / b)$ is not 3-connected. Let $(X,\{b\}, Y)$ be a vertical 3-separation of $M$ with $Y \cup\{b\}$ closed. Let $K$ denote the set of elements which are removable with respect to $B$. Then either:

(i) $|X \cap K| \geq 2$ and $|Y \cap K| \geq 2$, or

(ii) $X \cap K=\left\{b_{x}\right\},|Y \cap K| \geq 3$, and there exists $r \in Y \cap K$ such that $X \cup\{r\}$ is a 4-point coline, or

(iii) $|X \cap K| \geq 3, Y \cap K=\left\{b_{y}\right\}$, and there exists $r \in X \cap K$ such that $(Y-\operatorname{cl}(X)) \cup\{r\}$ is a 4-point coline.

Proof. Note that the vertical 3-separation $(X,\{b\}, Y)$ satisfies the hypotheses of Theorem 4.1.2. Applying the theorem, either $|X \cap K| \geq 2$ or $|X \cap K|=1$. Assume $|X \cap K|=1$. Then $X$ is a triad, there exists $r \in Y \cap K$ such that $X \cup\{r\}$ is a 4-point coline with $|(X \cup\{r\}) \cap B|=2$, and $(Y \cup\{b\})-\{r\}$ is closed. If $b \notin \mathrm{cl}(Y-\{r\})$, then by Lemma 2.5.4, $b \in \mathrm{cl}^{*}(X \cup\{r\})$, so that $X \cup\{b, r\}$ is a 5 -point coline; but then $\operatorname{si}(M / b)$ is 3-connected by the dual of Lemma 3.2.2: a contradiction. So $b \in \operatorname{cl}(Y-\{r\})$.

Suppose $r(Y-\{r\})=2$. If $|Y-\{r\}|=2,|Y|=3$, and as $(\operatorname{cl}(X)-$ $\{b\},\{b\}, Y-\operatorname{cl}(X))$ is a vertical 3-separation by Lemma 3.3.6, it must be the case that $Y-\operatorname{cl}(X)=Y$, so that $X \cup\{b\}$ is closed and $Y$ is a triad. But now $(Y-\{r\}) \cup\{b\}$ is a triangle, and $Y \cup\{b\}$ is a contradictory 4-element fan. So if $r(Y-\{r\})=2$, we must have $|Y-\{r\}| \geq 3$. As $b \in \operatorname{cl}(Y-\{r\})$, $|(Y-\{r\}) \cap(E(M)-B)| \geq 2$. Thus $|(Y-\{r\}) \cap K| \geq 2$ by Lemma 3.2.2. So $|Y \cap K| \geq 3$ and (ii) holds.

Now suppose that $r(Y-\{r\}) \geq 3$. By Lemma 3.2.8, $(X \cup\{r\},\{b\}, Y-\{r\})$ is a vertical 3-separation of $M$, therefore by Lemma 3.3.6, $(\operatorname{cl}(X \cup\{r\})-$ 
$\{b\},\{b\}, Y-\operatorname{cl}(X \cup\{r\}))$ is also. Note that $\operatorname{cl}(\operatorname{cl}(X \cup\{r\})-\{b\}) \cup\{b\}=$ $\operatorname{cl}(X \cup\{r\})$ is closed. Now applying Theorem 4.1.2, if 4.1.2 (i) holds, then $|(Y-\operatorname{cl}(X \cup\{r\})) \cap K| \geq 2$, so that $|Y \cap K| \geq 3$ : (ii) is satisfied and the theorem holds. So assume that 4.1 .2 (ii) holds. We have $\mid(Y-\operatorname{cl}(X \cup$ $\{r\})) \cap K \mid=1, Y-\operatorname{cl}(X \cup\{r\})$ is a triad, and there exists some $r^{\prime} \in$ $(\operatorname{cl}(X \cup\{r\})-\{b\}) \cap K$ such that $(Y-\operatorname{cl}(X \cup\{r\})) \cup\left\{r^{\prime}\right\}$ is a 4-point coline containing exactly two basis elements. Assume that $r^{\prime} \in X \cup\{r\}$. Then, as $r^{\prime} \in K$, we have two 4-point colines $X \cup\{r\}$ and $(Y-\operatorname{cl}(X \cup\{r\})) \cup\left\{r^{\prime}\right\}$ such that $|(X \cup\{r\}) \cap B|=\left|\left((Y-\operatorname{cl}(X \cup\{r\})) \cup\left\{r^{\prime}\right\}\right) \cap B\right|=2$ and $(X \cup\{r\}) \cap\left((Y-\operatorname{cl}(X \cup\{r\})) \cup\left\{r^{\prime}\right\}\right)=\left\{r^{\prime}\right\} \in B$. This is a contradiction to Lemma 3.2.5. Thus $r^{\prime} \notin X \cup\{r\}$ and $r^{\prime} \in Y$, so that $|Y \cap K| \geq 3$ and (ii) holds.

Now assume that $|X \cap K| \geq 2$. If $|Y \cap K| \geq 2$, (i) holds. So assume that $|Y \cap K| \in\{0,1\} .(\operatorname{cl}(X)-\{b\},\{b\}, Y-\operatorname{cl}(X))$ is a vertical 3-separation by Lemma 3.3.6 with $(\operatorname{cl}(X)-\{b\}) \cup\{b\}=\operatorname{cl}(X)$ closed. Apply Theorem 4.1.2. If 4.1 .2 (i) holds, $|(Y-\operatorname{cl}(X)) \cap K| \geq 2$. But we're working under the assumption that $|Y \cap K| \leq 1$. So 4.1 .2 (ii) holds. Then $|(Y-\operatorname{cl}(X)) \cap K|=1$, and there exists some $\rho \in(\operatorname{cl}(X)-\{b\}) \cap K$ such that $(Y-\operatorname{cl}(X)) \cup\{\rho\}$ is a 4-point coline containing exactly two basis elements. Now $|Y \cap K|=1$, so $\rho \in X$. If $|X \cap K| \geq 3$, (iii) holds. So we may assume that $|X \cap K|=2$. By Lemma 3.2.8, both $X-\{\rho\}$ and $(X-\{\rho\}) \cup\{b\}$ are exactly 3-separating. So $b \in \mathrm{cl}^{(*)}(X-\{\rho\})$ by Lemma 3.2.7. Now $b \in \operatorname{cl}(Y) \subset \operatorname{cl}(Y \cup\{\rho\})$, so $b \notin \operatorname{cl}^{*}(X-\{\rho\})$ by Lemma 2.5.4. Thus $b \in \operatorname{cl}(X-\{\rho\})$.

Suppose $r(X-\{\rho\})=2$. If $|X-\{\rho\}| \geq 3$, then $(X-\{\rho\}) \cup\{b\}$ is a 4-point line. This implies that $|((X-\{\rho\}) \cup\{b\}) \cap(E(M)-B)| \geq 2$ by Lemma 2.3.3, so $|((X-\{\rho\}) \cup\{b\}) \cap K| \geq 2$, hence $|(X-\{\rho\}) \cap K| \geq 2$ and $|X \cap K| \geq 3$ giving (iii). Otherwise, $|X-\{\rho\}|=2$. In this case, $(X-\{\rho\}) \cup\{b\}$ is a triangle, whereas $X$ is a triad; giving a 4-element fan $X \cup\{b\}$ in $M$; a contradiction.

Finally, suppose $r(X-\{\rho\}) \geq 3$. Then $(X-\{\rho\},\{b\}, Y \cup\{\rho\})$ is a vertical 3-separation. By Lemma 3.3.6, $(X-\operatorname{cl}(Y \cup\{\rho\}),\{b\}, \operatorname{cl}(Y \cup\{\rho\})-\{b\})$ is a vertical 3-separation also. Here $\operatorname{cl}((\operatorname{cl}(Y \cup\{\rho\})-\{b\}) \cup\{b\}))=\operatorname{cl}(Y \cup$ $\{\rho\})$ is closed. Applying Theorem 4.1.2 and noting that $|X \cap K|=2$ and $|(X-\{\rho\}) \cap K|=1$, it follows that 4.1 .2 (ii) holds. That is: $X-\operatorname{cl}(Y \cup\{\rho\})$ is a triad, and there exists some $\rho^{\prime} \in(\operatorname{cl}(Y \cup\{\rho\})-\{b\}) \cap K$ such that $(X-\operatorname{cl}(Y \cup\{\rho\})) \cup\left\{\rho^{\prime}\right\}$ is a 4-point coline containing exactly two basis elements. Note that $\rho^{\prime} \in K,|X \cap K|=2$, and $|Y \cap K|=\mid(Y-\operatorname{cl}(X)) \cap$ 
$K \mid=1$. Therefore either $\rho^{\prime}=\rho$ or $\rho^{\prime} \in Y-\operatorname{cl}(X)$. But in either case $(Y-\operatorname{cl}(X)) \cup\{\rho\}$ and $(X-\operatorname{cl}(Y \cup\{\rho\})) \cup\left\{\rho^{\prime}\right\}$ are two 4-point colines such that $|((Y-\operatorname{cl}(X)) \cup\{\rho\}) \cap B|=\left|\left((X-\operatorname{cl}(Y \cup\{\rho\})) \cup\left\{\rho^{\prime}\right\}\right) \cap B\right|=2$, and $((Y-\operatorname{cl}(X)) \cup\{\rho\}) \cap\left((X-\operatorname{cl}(Y \cup\{\rho\})) \cup\left\{\rho^{\prime}\right\}\right) \subset B$. This contradicts Lemma 3.2.5. We conclude that the theorem holds.

We obtain the following as a corollary.

Corollary 4.2.2. Let $M$ be a 3-connected matroid with no 4-element fans. Let $B$ be a basis of $M$ such that for some $c \in(E(M)-B), \operatorname{co}(M \backslash c)$ is not 3-connected. Let $(S,\{c\}, T)$ be a cyclic 3-separation of $M$ with $T \cup\{c\}$ coclosed. Let $K$ denote the set of elements which are removable with respect to $B$. Then either:

(i) $|S \cap K| \geq 2$ and $|T \cap K| \geq 2$, or

(ii) $S \cap K=\left\{c_{s}\right\},|T \cap K| \geq 3$, and there exists $r \in T \cap K$ such that $S \cup\{r\}$ is a 4-point line, or

(iii) $|S \cap K| \geq 3, T \cap K=\left\{c_{t}\right\}$, and there exists $r \in S \cap K$ such that $\left(T-\mathrm{cl}^{*}(\bar{S})\right) \cup\{r\}$ is a 4-point line.

Proof. This follows by dualising Theorem 4.2.1 and using Lemmas 3.3.3, 3.5.3, and 3.5.5.

Suppose $M$ is a 3 -connected matroid with no 4-element fans, $B$ a basis of $M$, and let $K$ denote the set of elements of $M$ which are removable with respect to $B$. Theorem 4.2.1 states that if there exists some $b \in B-K$, then $|K| \geq 4$. Also, Corollary 4.2.2 states that if there exists some $c \in$ $(E(M)-B)-K$, then $|K| \geq 4$. Putting these results together we obtain the following:

Proof of Theorem 4.0.1. Suppose $|K| \leq 3$. Then by Theorem 4.2.1 and Corollary 4.2.2, $B-K=(E(M)-B)-K=\emptyset$. But then $B \cup(E(M)-B)=$ $E(M)=K$ so that $|E(M)| \leq 3$. 


\section{Chapter 5}

\section{Matroids with Minimal Removability}

In light of the results in preceding chapter, we have that for any matroid $M$ on a ground set of size at least four, and for any basis $B$ of $M$, provided that $M$ does not contain any four element fans, we can always find at least four elements which are removable with respect to $B$. We now turn our attention to the cases for which there exist exactly four elements which are removable with respect to $B$. We begin with a definition.

Definition 5.0.1. A matroid $M$ has path-width 3 if there is an ordering $\left(e_{1}, \ldots, e_{n}\right)$ of $E(M)$ such that, for all $i \in\{1, \ldots, n\}, \lambda\left(\left\{e_{1}, \ldots, e_{i}\right\}\right) \leq 2$

The class of matroids with path-width 3 has been studied in detail, and as a result, the structure of matroids which belong to this class is well understood. See Hall, Oxley, and Semple [6] for a detailed examination of this class of matroids. The primary result of this chapter is the following:

Theorem 5.0.2. Let $M$ be a 3-connected matroid with no 4-element fans, and let $B$ be a basis of $M$. Let $K$ denote the set of elements which are removable with respect to $B$. If $|K|=4$, then $M$ has path-width 3 .

\subsection{Preliminary Constructions}

Proposition 5.1.1. Let $M$ be a 3-connected matroid with no 4-element fans. Let $B$ be a basis of $M$ such that for some $b \in B, \operatorname{si}(M / b)$ is not 3-connected. 
Let $K$ denote the set of elements which are removable with respect to $B$. Suppose $|K|=4$. Then, provided $M$ is not the union of a 4-point line and a 4-point coline, there exists a vertical 3-separation $(X,\{b\}, Y)$ of $M$ such that $|X \cap K|=2,|Y \cap K|=2$, and $Y \cup\{b\}$ is closed.

Proof. Let $\left(X^{\prime},\{b\}, Y^{\prime}\right)$ be a vertical 3-separation of $M$ with $Y^{\prime} \cup\{b\}$ closed. If $\left|X^{\prime} \cap K\right|=\left|Y^{\prime} \cap K\right|=2$, the result is immediate. So assume that this isn't the case. Now either 4.2.1 (ii) or 4.2 .1 (iii) holds.

Suppose (ii) holds. Then $X^{\prime} \cap K=\left\{b_{x}\right\},\left|Y^{\prime} \cap K\right| \geq 3$, and there exists some $r \in Y^{\prime} \cap K$ such that $X^{\prime} \cup\{r\}$ is a 4-point coline. By Lemma 3.4.3, either $\left(X^{\prime} \cup\{r\},\{b\}, Y^{\prime}-\{r\}\right)$ is a vertical 3-separation of $M$, or $r\left(\left(Y^{\prime}-\{r\}\right) \cup\{b\}\right)=$ 2. Suppose the latter holds. If $\left|Y^{\prime}-\{r\}\right| \geq 4$, then $\mid\left(\left(Y^{\prime}-\{r\}\right) \cup\{b\}\right) \cap$ $K \mid \geq 3$ by Lemmas 2.3.3 and 3.2.2. But then $\left|Y^{\prime} \cap K\right| \geq 4$; which is absurd. If $\left|Y^{\prime}-\{r\}\right|=3$, then $\left(Y^{\prime}-\{r\}\right) \cup\{b\}$ is a 4-point line. But $E-\left(\left(Y^{\prime}-\{r\}\right) \cup\{b\}\right)=X \cup\{r\}$ is a 4-point coline, so that $M$ is the union of a 4-point line and a 4-point coline, which is not the case. So it must be that $\left|Y^{\prime}-\{r\}\right|=2$. Here $\left|Y^{\prime}\right|=3$. As $\left(\operatorname{cl}\left(X^{\prime}\right)-\{b\},\{b\}, Y^{\prime}-\operatorname{cl}\left(X^{\prime}\right)\right)$ is a vertical 3-separation of $M$ by Lemma 3.3.6, it must be that $\operatorname{cl}\left(X^{\prime}-\{b\}\right) \cup$ $\{b\}=\operatorname{cl}\left(X^{\prime}\right)=X^{\prime}$ is closed. Therefore, $Y^{\prime}$ is a triad that meets the triangle $\left(Y^{\prime}-\{r\}\right) \cup\{b\}$; giving a 4-element fan $Y^{\prime} \cup\{b\}$ in $M$; a contradiction. So $\left(X^{\prime} \cup\{r\},\{b\}, Y^{\prime}-\{r\}\right)$ must be a vertical 3-separation of $M$. As $X^{\prime} \cup\{r\}$ is a 4-point coline, $x \in \operatorname{cl}^{*}\left(\left(X^{\prime} \cup\{r\}\right)-\{x\}\right)$ for all $x \in X^{\prime} \cup\{r\}$. Hence $\operatorname{cl}\left(\left(Y^{\prime}-\{r\}\right) \cup\{b\}\right) \cap\left(X^{\prime} \cup\{r\}\right)=\emptyset$ and $\left(Y^{\prime}-\{r\}\right) \cup\{b\}$ is closed. Letting $Y=Y^{\prime}-\{r\}$ and $X=X^{\prime} \cup\{r\}$, the result follows.

Now suppose 4.2 .1 (iii) holds. Then $\left|X^{\prime} \cap K\right| \geq 3, Y^{\prime} \cap K=\left\{b_{y}\right\}$, and there exists some $r \in X^{\prime} \cap K$ such that $\left(Y^{\prime}-\operatorname{cl}\left(X^{\prime}\right)\right) \cup\{r\}$ is a 4-point coline. Now $\left(\operatorname{cl}\left(X^{\prime}\right)-\{b\},\{b\}, Y^{\prime}-\operatorname{cl}\left(X^{\prime}\right)\right)$ is a vertical 3-separation of $M$ by Lemma 3.3.6. By Lemma 3.4.3, either $\left(\operatorname{cl}\left(X^{\prime}\right)-\{b, r\},\{b\},\left(Y^{\prime}-\operatorname{cl}\left(X^{\prime}\right)\right) \cup\right.$ $\{r\})$ is a vertical 3-separation, or $r\left(\left(\operatorname{cl}\left(X^{\prime}\right)-\{b, r\}\right) \cup\{b\}\right)=2$. Suppose $r\left(\left(\operatorname{cl}\left(X^{\prime}\right)-\{b, r\}\right) \cup\{b\}\right)=2$. If $\left|\operatorname{cl}\left(X^{\prime}\right)-\{b, r\}\right|=2$, then $\left|\operatorname{cl}\left(X^{\prime}\right)-\{b\}\right|=3$ and so $\operatorname{cl}\left(X^{\prime}\right)-\{b\}=X^{\prime}$. As $Y^{\prime} \cup\{b\}$ is closed, $X^{\prime}$ is a triad, whereas $\left(X^{\prime}-\{r\}\right) \cup\{b\}$ is a triangle. This gives a contradictory 4-element fan $X^{\prime} \cup\{b\}$ in $M$. If $\left|\operatorname{cl}\left(X^{\prime}\right)-\{b, r\}\right|=3$, then $\operatorname{cl}\left(X^{\prime}\right)-\{r\}$ is a 4-point line, whereas $\left(Y^{\prime}-\operatorname{cl}\left(X^{\prime}\right)\right) \cup\{r\}$ is a 4-point coline. But $M$ is not the union of a 4-point line and a 4-point coline. Finally, if $\left|\operatorname{cl}\left(X^{\prime}\right)-\{b, r\}\right| \geq 4$, then $\operatorname{cl}\left(X^{\prime}\right)-\{r\}$ is a rank 2 set containing at least five elements. Therefore $\left|\left(\operatorname{cl}\left(X^{\prime}\right)-\{r\}\right) \cap K\right| \geq 3$ by Lemmas 2.3.3 and 3.2.2. As $b_{y} \in Y^{\prime}-\operatorname{cl}\left(X^{\prime}\right), b_{y} \notin\left(\operatorname{cl}\left(X^{\prime}\right)-\{r\}\right) \cap K$. 
Thus $\left(\operatorname{cl}\left(X^{\prime}\right)-\{r\}\right) \cap K \subset X^{\prime}$, and $\left|X^{\prime} \cap K\right| \geq 4$; a contradiction. It must be that $\left(\operatorname{cl}\left(X^{\prime}\right)-\{b, r\},\{b\},\left(Y^{\prime}-\operatorname{cl}\left(X^{\prime}\right)\right) \cup\{r\}\right)$ is a vertical 3-separation of $M$. Moreover, as $r \in \mathrm{cl}^{*}\left(Y^{\prime}-\operatorname{cl}\left(X^{\prime}\right)\right), r \notin \operatorname{cl}\left(\left(\operatorname{cl}\left(X^{\prime}\right)-\{b, r\}\right) \cup\{b\}\right)$. Also, $\left(Y^{\prime}-\operatorname{cl}\left(X^{\prime}\right)\right) \cap \operatorname{cl}\left(\left(\operatorname{cl}\left(X^{\prime}\right)-\{b, r\}\right) \cup\{b\}\right)=\emptyset$. Therefore, $\left(\operatorname{cl}\left(X^{\prime}\right)-\{b, r\}\right) \cup\{b\}$ is closed. Letting $X=\left(Y^{\prime}-\operatorname{cl}\left(X^{\prime}\right)\right) \cup\{r\}$ and $Y=\operatorname{cl}\left(X^{\prime}\right)-\{b, r\}$, we conclude that the theorem holds.

Let $M$ be a 3-connected matroid, let $B$ be a basis of $M$, and let $K$ denote the set of elements of $E(M)$ which are removable with respect to $B$. We shall call a vertical (cyclic) 3-separation $(X,\{e\}, Y)$ of $M$ balanced whenever $|X \cap K|=|Y \cap K|=2$.

The following is a simple restatement of Proposition 5.1.1 using this terminology.

Corollary 5.1.2. Let $M$ be a 3-connected matroid with no 4-element fans, and let $B$ be a basis of $M$. Let $K$ denote the set of elements which are removable with respect to $B$, and suppose $|K|=4$. If $M$ is not the union of a 4-point line and a 4-point coline, then for each $b_{i} \in B-K$, there exists a balanced vertical 3-separation $\left(X_{i},\left\{b_{i}\right\}, Y_{i}\right)$ such that $Y_{i} \cup\left\{b_{i}\right\}$ is closed.

Corollary 5.1.3. Let $M$ be a 3-connected matroid with no 4-element fans, and let $B$ be a basis of $M$. Let $K$ denote the set of elements which are removable with respect to $B$, and suppose $|K|=4$. If $M$ is not the union of a 4-point line and a 4-point coline, then for each $c_{i} \in(E(M)-B)-K$, there exists a balanced cyclic 3-separation $\left(S_{i},\left\{c_{i}\right\}, T_{i}\right)$ such that $T_{i} \cup\left\{c_{i}\right\}$ is coclosed.

Proof. This follows from the dual of Corollary 5.1.2 and the use of Lemmas 3.5.3, and 3.5.5.

Corollary 5.1.2 and Corollary 5.1.3 above are used freely throughout the remainder of this thesis.

Corollary 5.1.4. Let $M$ be a 3-connected matroid with no 4-element fans, and let $B$ be a basis of $M$. Let $K$ denote the set of elements which are removable with respect to $B$, and suppose $|K|=4$. If $|K \cap(E(M)-B)| \geq 3$, then for every $b \in B-K$, every vertical 3-separation $(X,\{b\}, Y)$ of $M$ is balanced. 
Proof. Suppose $|K \cap(E(M)-B)| \geq 3$ and $b \in B-K$. Let $(X,\{b\}, Y)$ be a vertical 3-separation of $M$ that is not balanced. Then we can assume that $|X \cap K|=1$. Now $(X-\operatorname{cl}(Y),\{b\}, \operatorname{cl}(Y)-\{b\})$ is also a vertical 3-separation of $M$ by Lemma 3.3.6, and, by Theorem 4.1.2, $|(X-\operatorname{cl}(Y)) \cap K|=1$, and there exists some $r \in \operatorname{cl}(Y) \cap K$ such that $(X-\operatorname{cl}(Y)) \cup\{r\}$ is a 4-point coline. But then $|((X-\operatorname{cl}(Y)) \cup\{r\}) \cap B \cap K| \geq 2$ by Lemmas 2.5.6 and 3.2.3. This contradicts the fact that $|K \cap B| \in\{0,1\}$. Therefore $(X,\{b\}, Y)$ is balanced.

Lemma 5.1.5. Let $M$ be a 3-connected matroid with no 4-element fans, and let $B$ be a basis of $M$. Let $K$ denote the set of elements which are removable with respect to $B$, and suppose $|K|=4$. Then:

(i) if $L$ is a line of $M$ and there exists some $b \in B-K$, then $|L| \leq 4$, and

(ii) if $\Gamma$ is a coline of $M$ and there exists some $c \in(E(M)-B)-K$, then $|\Gamma| \leq 4$.

Proof. Suppose $b \in B-K$ and let $L$ be a line of $M$ with $|L| \geq 5$. Then $\mid L \cap$ $(E(M)-B) \cap K \mid \geq 3$ by Lemmas 3.2.2 and 2.3.3. Let $(X,\{b\}, Y)$ be a vertical 3 -separation of $M$. We can assume without loss of generality that $|L \cap Y| \geq 2$. By Lemma 3.3.6, $(X-\operatorname{cl}(Y),\{b\}, \operatorname{cl}(Y)-\{b\})$ is a vertical 3-separation of $M$, and we have $L \cap(E(M)-B) \cap K \subset \operatorname{cl}(Y)-\{b\}$. So $(X-\operatorname{cl}(Y),\{b\}, \operatorname{cl}(Y)-\{b\})$ is not balanced. But this contradicts Lemma 5.1.4. Therefore $|L| \leq 4$ and (i) holds. By dualising this result and using Lemma 3.5.3, we obtain (ii).

Proposition 5.1.6. Let $M$ be a 3-connected matroid with no 4-element fans, and let $B$ be a basis of $M$. Let $K$ denote the set of elements which are removable with respect to $B$, and suppose $|K|=4$. Let $\Gamma_{1}, \Gamma_{2}$ be distinct 4-point colines of $M$. If there exists some $c \in(E(M)-B)-K$, then $\mid \Gamma_{1} \cap$ $\Gamma_{2} \cap B \mid \neq 1$.

Proof. Suppose that $c \in(E(M)-B)-K$ and $\left|\Gamma_{1} \cap \Gamma_{2} \cap B\right|=1$. By Lemma 2.5.6, $\left|\Gamma_{1} \cap B\right| \geq 2$ and $\left|\Gamma_{2} \cap B\right| \geq 2$. If $\left|\Gamma_{1} \cap \Gamma_{2}\right| \geq 2$, then $r^{*}\left(\Gamma_{1} \cap \Gamma_{2}\right) \geq 2$ as $M$ is 3-connected, and so, by Lemma 2.5.7, $\Gamma_{1} \cup \Gamma_{2}$ is a coline containing at least five elements. But this contradicts Lemma 5.1.5. So we may assume that $\Gamma_{1} \cap \Gamma_{2}=\Gamma_{1} \cap \Gamma_{2} \cap B$.

By Lemma 3.2.5, it cannot be the case that $\left|\Gamma_{1} \cap B\right|=\left|\Gamma_{2} \cap B\right|=2$. So we can assume without loss of generality that $\left|\Gamma_{1} \cap B\right| \geq 3$. Now $\left|\left(\Gamma_{1} \cup \Gamma_{2}\right) \cap B\right|=$ 
$\left|\left(\Gamma_{1} \cap B\right) \cup\left(\Gamma_{2} \cap B\right)\right|=\left|\Gamma_{1} \cap B\right|+\left|\Gamma_{2} \cap B\right|-\left|\Gamma_{1} \cap \Gamma_{2} \cap B\right| \geq 4$. As $|K|=4$, and every element of $\left(\Gamma_{1} \cup \Gamma_{2}\right) \cap B$ is removable with respect to $B$, it must be that $\left|\left(\Gamma_{1} \cup \Gamma_{2}\right) \cap B\right|=4$ and $K=\left(\Gamma_{1} \cup \Gamma_{2}\right) \cap B$. In particular, note that $K \subseteq B$.

Now consider the matroid $M^{*}$ with basis $E(M)-B$. As $M$ is 3-connected, $M^{*}$ is also 3-connected. Also, $M^{*}$ contains no 4-element fans by Lemma 3.5.5. By Lemma 3.5.3, the set of elements removable with respect to $E(M)-B$ is equal to $K . \Gamma_{1}$ and $\Gamma_{2}$ are 4-point lines of $M^{*}$ which meet at exactly one point $\alpha \in K$. Note that $r^{*}\left(\Gamma_{1} \cup \Gamma_{2}\right)=3$. Consider the element $c \in E(M)-B$. As $c \notin K, \operatorname{si}\left(M^{*} / c\right)$ is not 3-connected. By Lemmas 3.3.12 and 3.3.6, there exists a vertical 3-separation $(P,\{c\}, Q)$ of $M^{*}$ with $Q \cup\{c\}$ closed. Recall that $\left|\Gamma_{1} \cap K\right|=3$ and $K \subseteq B$. Note that $B$ is now a cobasis of the matroid $M^{*}$ so that Corollary 5.1.4 may be used freely when studying $M^{*}$. If $\left|\Gamma_{1} \cap Q\right| \geq 2$, then $\Gamma_{1} \subseteq Q \cup\{c\}$ as $Q \cup\{c\}$ is closed. But then $|(Q \cup\{c\}) \cap K| \geq 3$ which contradicts Lemma 5.1.4. Therefore $\left|\Gamma_{1} \cap Q\right| \leq 1$. So $\left|\Gamma_{1} \cap P\right| \geq 2$. By Lemma 3.3.6, $(\operatorname{cl}(P)-\{c\},\{c\}, Q-\operatorname{cl}(P))$ is a vertical 3-separation of $M^{*}$. But now $\Gamma_{1} \subset \operatorname{cl}(P)-\{c\}$ so that $(\operatorname{cl}(P)-\{c\},\{c\}, Q-\operatorname{cl}(P))$ is not balanced; contradicting Corollary 5.1.4. We conclude that the proposition holds.

Lemma 5.1.7. Let $M$ be a 3-connected matroid with no 4-element fans and let $B$ be a basis of $M$. Let $K$ denote the set of elements which are removable with respect to $B$, and suppose $|K|=4$. If $b \in B-K$ and $(X,\{b\}, Y)$ is a balanced vertical 3-separation of $M$ with $Y \cup\{b\}$ closed, then for any $w \in X \cap \mathrm{cl}^{*}(Y)$, either:

(i) $(X-\{w\},\{b\}, Y \cup\{w\})$ is a vertical 3-separation of $M$, or

(ii) $(X-\{w\}) \cup\{b\}$ is a 4-point line.

Proof. Let $w$ be an element of $X \cap \mathrm{cl}^{*}(Y)$. Suppose $r((X-\{w\}) \cup\{b\})=2$. If $|(X-\{w\}) \cup\{b\}| \geq 5$, then $|(X-\{w\}) \cap K| \geq 3$ by Lemmas 2.3.3 and 3.2.2; but this contradicts the fact that $(X,\{b\}, Y)$ is balanced. If $\mid(X-\{w\}) \cup$ $\{b\} \mid=3$, then $|X|=3$, so that $X$ is a triad and $X \cup\{b\}$ is a contradictory 4-element fan. Therefore $r((X-\{w\}) \cup\{b\})=2 \Longrightarrow|(X-\{w\}) \cup\{b\}|=$ 4; giving (ii). Otherwise, $r((X-\{w\}) \cup\{b\}) \geq 3$ and by Lemma 3.4.3, $(X-\{w\},\{b\}, Y \cup\{w\})$ is a vertical 3-separation of $M$. 
Let $M$ be a 3-connected matroid, $B$ a basis of $M$, and let $K$ denote the set of elements which are removable with respect to $B$. We shall call a vertical (cyclic) 3-separation $(X,\{b\}, Y)$ of $M$ colinearly balanced if it is balanced, and, for any 4-point coline $\Gamma \subset E(M), \Gamma \subset X$ or $\Gamma \subset Y$. Similarly, we shall call a vertical (cyclic) 3-separation $(X,\{b\}, Y)$ linearly balanced if it is balanced, and, for any 4-point line $L \subset E(M), L \subset X$ or $L \subset Y$.

Let $M$ be a matroid, and $B$ be a basis of $M$. We shall call $M$ colinearly entwined if there exists 4-point colines $\Gamma_{1}, \Gamma_{2} \subset E(M)$ such that $\left|\Gamma_{1} \cap \Gamma_{2}\right|=1$ where $\Gamma_{1} \cap \Gamma_{2} \subseteq E(M)-B$.

Proposition 5.1.8. Let $M$ be a 3-connected matroid with no 4-element fans, and let $B$ be a basis of $M$. Suppose that $M$ is colinearly entwined with respect to the 4-point colines $\Gamma_{1}, \Gamma_{2} \subset E(M)$. Let $K$ denote the set of elements which are removable with respect to $B$, and suppose $|K|=4$. Then for all $b \in B-K$, there exists a balanced vertical 3-separation $\left(X^{\prime},\{b\}, Y^{\prime}\right)$ of $M$ with $\Gamma_{1} \subseteq X^{\prime}$ and $\Gamma_{2}-\Gamma_{1} \subseteq Y^{\prime}$.

Proof. Let $\Gamma_{1} \cap \Gamma_{2}=\{\alpha\}$. Then $\alpha \in E(M)-B$. It follows from Lemma 3.2.3 and Lemma 2.5.6 that $K \subset \Gamma_{1} \cup \Gamma_{2}, \alpha \notin K$, and $M$ does not contain any 4-point colines apart from $\Gamma_{1}$ and $\Gamma_{2}$. Suppose $b \in B-K$, and let $(X,\{b\}, Y)$ be a balanced vertical 3-separation of $M$ with $Y \cup\{b\}$ closed. We construct a balanced vertical 3 -separation that satisfies the theorem through a case analysis of the possible sizes of the set $\Gamma_{1} \cap Y$.

\section{Case (i): $\left|\Gamma_{1} \cap Y\right|=4$}

Suppose $\Gamma_{1} \cap X=\emptyset$. Then $\left|\Gamma_{2} \cap X \cap K\right|=2$. Hence $\Gamma_{2} \subseteq \mathrm{cl}^{*}(X)$. Note that $\left|\left(\Gamma_{1} \cap Y\right)-\Gamma_{2}\right|=3$ so that $r\left(Y-\Gamma_{2}\right) \geq 3$ by Lemma 3.2.4. Applying Lemma 3.4.3, it follows that $\left(X \cup \Gamma_{2},\{b\}, Y-\Gamma_{2}\right)$ is a suitable balanced vertical 3-separation of $M$.

$$
\text { Case (ii): }\left|\Gamma_{1} \cap Y\right|=3
$$

Suppose $\Gamma_{1} \cap X=\{z\}$. Then $(X-\{z\},\{b\}, Y \cup\{z\})$ is a vertical 3separation of $M$ by Lemma 5.1.7. If $(X-\{z\},\{b\}, Y \cup\{z\})$ is not balanced, then by Theorem 4.1.2, $X-\{z\}$ contains a triad $\Lambda$ and there exists some $r \in(Y \cup\{z\}) \cap B$ such that $\Lambda \cup\{r\}$ is a 4-point coline. But then it must be that $\Lambda \cup\{r\}=\Gamma_{2}$ and $r=\alpha$ which is absurd as $\alpha \notin B$. So $(X-$ 
$\{z\},\{b\}, Y \cup\{z\})$ must be balanced. Then $\left|(X-\{z\}) \cap \Gamma_{2} \cap K\right|=2$. So $\Gamma_{2} \subseteq \mathrm{cl}^{*}(X-\{z\})$. Now $\Gamma_{1}-\{\alpha\} \subseteq(Y \cup\{z\})-\Gamma_{2}$, hence $r((Y \cup\{z\})-$ $\left.\Gamma_{2}\right) \geq 3$ by Lemma 3.2.4. Therefore, applying Lemma 3.4.3, it must be that $\left((X-\{z\}) \cup\left(\Gamma_{2}-\{\alpha\}\right),\{b\},(Y \cup\{z\})-\left(\Gamma_{2}-\{\alpha\}\right)\right)$ is a vertical 3-separation of $M$. Letting $X^{\prime}=(Y \cup\{z\})-\left(\Gamma_{2}-\{\alpha\}\right)$ and $Y^{\prime}=(X-\{z\}) \cup\left(\Gamma_{2}-\{\alpha\}\right)$, the proposition follows.

Case (iii): $\left|\Gamma_{1} \cap Y\right|=2$

Suppose $\Gamma_{1} \cap X=\left\{z_{1}, z_{2}\right\}$. By Lemma 5.1.7, $\left(X-\left\{z_{1}\right\},\{b\}, Y \cup\left\{z_{1}\right\}\right)$ is a vertical 3-separation of $M$. Applying Lemma 3.3.6, $(X-\operatorname{cl}(Y \cup$ $\left.\left\{z_{1}\right\},\{b\}, \operatorname{cl}\left(Y \cup\left\{z_{1}\right\}\right)-\{b\}\right)$ is also a vertical 3 separation of $M$. As $z_{2} \in \operatorname{cl}^{*}\left(Y \cup\left\{z_{1}\right\}\right)$, Lemma 3.3.7 implies that $z_{2} \notin \operatorname{cl}\left(Y \cup\left\{z_{1}\right\}\right)$.

If $\left(X-\operatorname{cl}\left(Y \cup\left\{z_{1}\right\}\right),\{b\}, \operatorname{cl}\left(Y \cup\left\{z_{1}\right\}\right)-\{b\}\right)$ is not balanced, then by Theorem 4.1.2, $X-\operatorname{cl}\left(Y \cup\left\{z_{1}\right\}\right)$ is a triad and there exists some $r \in \operatorname{cl}(Y \cup$ $\left.\left\{z_{1}\right\}\right)-\{b\}$ such that $\left(X-\operatorname{cl}\left(Y \cup\left\{z_{1}\right\}\right)\right) \cup\{r\}$ is a 4-point coline. Now $z_{2} \in\left(X-\operatorname{cl}\left(Y \cup\left\{z_{1}\right\}\right)\right) \cup\{r\}$ and $z_{2} \in \Gamma_{1}$, so $z_{2}=\alpha$ and $r \notin \Gamma_{1}$. Therefore $r\left(\operatorname{cl}\left(Y \cup\left\{z_{1}\right\}\right)-\{b, r\}\right) \geq 3$ by Lemma 3.2.4, and so, by an application of Lemma 3.4.3, it must be that $\left(\left(X-\operatorname{cl}\left(Y \cup\left\{z_{1}\right\}\right)\right) \cup\{r\},\{b\}, \operatorname{cl}\left(Y \cup\left\{z_{1}\right\}\right)-\right.$ $\{b, r\})$ is a vertical 3-separation of $M$. Letting $X^{\prime}=\left(X-\operatorname{cl}\left(Y \cup\left\{z_{1}\right\}\right)\right) \cup\{r\}$ and $Y^{\prime}=\operatorname{cl}\left(Y \cup\left\{z_{1}\right\}\right)-\{b, r\}$, we obtain a suitable balanced vertical 3separation of $M$.

So assume that $\left(X-\operatorname{cl}\left(Y \cup\left\{z_{1}\right\}\right),\{b\}, \operatorname{cl}\left(Y \cup\left\{z_{1}\right\}\right)-\{b\}\right)$ is balanced. Noting that $\left(\operatorname{cl}\left(Y \cup\left\{z_{1}\right\}\right)-\{b\}\right) \cup\{b\}=\operatorname{cl}\left(Y \cup\left\{z_{1}\right\}\right)$ is closed, and applying Lemma 5.1.7, we have that $\left(X-\left(\operatorname{cl}\left(Y \cup\left\{z_{1}\right\}\right) \cup\left\{z_{2}\right\}\right),\{b\},\left(\operatorname{cl}\left(Y \cup\left\{z_{1}\right\}\right) \cup\right.\right.$ $\left.\left.\left\{z_{2}\right\}\right)-\{b\}\right)$ is a vertical 3-separation of $M$. If this separation is not balanced, then by Theorem 4.1.2, $X-\left(\operatorname{cl}\left(Y \cup\left\{z_{1}\right\}\right) \cup\left\{z_{2}\right\}\right)$ contains a triad $\Lambda$ and there exists some $r^{\prime} \in\left(\operatorname{cl}\left(Y \cup\left\{z_{1}\right\}\right) \cup\left\{z_{2}\right\}\right)-\{b\}$ where $r^{\prime} \in B$ such that $\Lambda \cup\left\{r^{\prime}\right\}$ is a 4-point coline. But then $\Lambda \cup\left\{r^{\prime}\right\}=\Gamma_{2}$ and $r^{\prime}=\alpha$. This contradicts the fact that $\alpha \notin B$. So $\left(X-\left(\operatorname{cl}\left(Y \cup\left\{z_{1}\right\}\right) \cup\left\{z_{2}\right\}\right),\{b\},\left(\operatorname{cl}\left(Y \cup\left\{z_{1}\right\}\right) \cup\right.\right.$ $\left.\left.\left\{z_{2}\right\}\right)-\{b\}\right)$ must be balanced. Hence $\left|\Gamma_{2} \cap\left(X-\left(\operatorname{cl}\left(Y \cup\left\{z_{1}\right\}\right) \cup\left\{z_{2}\right\}\right)\right)\right| \geq 2$. Therefore $\Gamma_{2} \in \operatorname{cl}^{*}\left(X-\left(\operatorname{cl}\left(Y \cup\left\{z_{1}\right\}\right) \cup\left\{z_{2}\right\}\right)\right)$. Noting that $r\left(\left(\operatorname{cl}\left(Y \cup\left\{z_{1}\right\}\right) \cup\right.\right.$ $\left.\left.\left\{z_{2}\right\}\right)-\left(\{b\} \cup \Gamma_{2}\right)\right) \geq 3$ by Lemma 3.2.4, we have that, by Lemma 3.4.3, $\left(\left(X-\left(\operatorname{cl}\left(Y \cup\left\{z_{1}\right\}\right) \cup\left\{z_{2}\right\}\right)\right) \cup \Gamma_{2},\{b\},\left(\left(Y \cup\left\{z_{1}\right\}\right) \cup\left\{z_{2}\right\}\right)-\left(\{b\} \cup \Gamma_{2}\right)\right)$ is a vertical 3-separation of $M$. Letting $X^{\prime}=\left(X-\left(\operatorname{cl}\left(Y \cup\left\{z_{1}\right\}\right) \cup\left\{z_{2}\right\}\right)\right) \cup \Gamma_{2}$ and $Y^{\prime}=\left(\left(Y \cup\left\{z_{1}\right\}\right) \cup\left\{z_{2}\right\}\right)-\left(\{b\} \cup \Gamma_{2}\right)$, the result follows. 
Case (iv): $\left|\Gamma_{1} \cap Y\right|=1$

Suppose $\Gamma_{1} \cap Y=\{z\}$. Then $z \in \mathrm{cl}^{*}(X)$ and so $z \notin \operatorname{cl}(X)$ by Lemma 3.3.7. Applying Lemma 3.3.6, $(\operatorname{cl}(X)-\{b\},\{b\}, Y-\operatorname{cl}(X))$ is a vertical 3-separation of $M$ with $z \in Y-\operatorname{cl}(X)$. If it isn't balanced, then by Theorem 4.1.2, $Y-\operatorname{cl}(X)$ is a triad and there exists some $r \in \operatorname{cl}(X)-\{b\}$ such that $(Y-$ $\operatorname{cl}(X)) \cup\{r\}$ is a 4-point coline. Then $(Y-\operatorname{cl}(X)) \cup\{r\}=\Gamma_{2}, z=\alpha$, and it must be that $r \notin \Gamma_{1}$ because $\left|\Gamma_{1} \cap \Gamma_{2}\right|=1$. So $r(\operatorname{cl}(X)-\{b, r\}) \geq$ 3 by Lemma 3.2.4. Now $r \in \mathrm{cl}^{*}(Y-\operatorname{cl}(X))$, so that, by Lemma 3.4.3, $(\operatorname{cl}(X)-\{b, r\},\{b\},(Y-\operatorname{cl}(X)) \cup\{r\})$ is a vertical 3-separation of $M$. Letting $X^{\prime}=(Y-\operatorname{cl}(X)) \cup\{r\}$ and $Y^{\prime}=\operatorname{cl}(X)-\{b, r\}$, we have $\left(X^{\prime},\{b\}, Y^{\prime}\right)$ as a suitable balanced vertical 3 -separation of $M$.

So assume that $(\operatorname{cl}(X)-\{b\},\{b\}, Y-\operatorname{cl}(X))$ is balanced. Then by Lemma 5.1.7, $((\operatorname{cl}(X) \cup\{z\})-\{b\},\{b\}, Y-(\operatorname{cl}(X) \cup\{z\}))$ is a vertical 3 -separation of $M$. If this 3 -separation is not balanced, then by Theorem 4.1.2, $Y-(\operatorname{cl}(X) \cup\{z\})$ contains a triad $\Lambda$ and there exists some $r^{\prime} \in(\operatorname{cl}(X) \cup\{z\})-\{b\}$ where $r^{\prime} \in B$ such that $\Lambda \cup\left\{r^{\prime}\right\}$ is a 4-point coline. But then $\Lambda \cup\left\{r^{\prime}\right\}=\Gamma_{2}$ and $r^{\prime}=\alpha$. This contradicts the fact that $\alpha \notin B$. Hence $((\operatorname{cl}(X) \cup\{z\})-\{b\},\{b\}, Y-(\operatorname{cl}(X) \cup\{z\}))$ must be balanced. Therefore $\left|(Y-(\operatorname{cl}(X) \cup\{z\})) \cap \Gamma_{2} \cap K\right|=2$ and $\Gamma_{2} \subseteq \operatorname{cl}^{*}(Y-(\operatorname{cl}(X) \cup\{z\}))$. Now $\left|\Gamma_{1} \cap\left((\operatorname{cl}(X) \cup\{z\})-\left(\{b\} \cup\left\{\Gamma_{2}\right\}\right)\right)\right|=3$, so that $r\left((\operatorname{cl}(X) \cup\{z\})-\left(\{b\} \cup\left\{\Gamma_{2}\right\}\right)\right) \geq 3$. With Lemma 3.4.3, we then deduce that $\left((\operatorname{cl}(X) \cup\{z\})-\left(\{b\} \cup\left\{\Gamma_{2}\right\}\right),\{b\},(Y-(\operatorname{cl}(X) \cup\{z\})) \cup \Gamma_{2}\right)$ is a vertical 3-separation of $M$. Let $X^{\prime}=(Y-(\operatorname{cl}(X) \cup\{z\})) \cup \Gamma_{2}$ and $Y^{\prime}=(\operatorname{cl}(X) \cup\{z\})-\left(\{b\} \cup\left\{\Gamma_{2}\right\}\right)$, and it follows that $\left(X^{\prime},\{b\}, Y^{\prime}\right)$ is a suitable balanced vertical 3 -separation of $M$.

$$
\text { Case (v): }\left|\Gamma_{1} \cap Y\right|=0
$$

Suppose $\Gamma_{1} \subseteq X$. Then $\left|\Gamma_{2} \cap Y \cap K\right|=2$, so that $\Gamma_{2} \subseteq \operatorname{cl}^{*}(Y)$. We have $r\left(X-\Gamma_{2}\right) \geq 3$ so an application of Lemma 3.4.3 gives a balanced vertical 3-separation $\left(X-\Gamma_{2},\{b\}, Y \cup \Gamma_{2}\right)$ that satisfies the requirements of the Proposition.

Proposition 5.1.9. Let $M$ be a 3-connected matroid with no 4-element fans, and let $B$ be a basis of $M$. Let $K$ denote the set of elements which are removable with respect to $B$. Suppose $|K|=4$ and that there exists some $c \in(E(M)-B)-K$. If $M$ is not colinearly entwined and $M$ is not the 
union of a 4-point line and a 4-point coline, then for each $b_{i} \in B-K$ such that $b_{i}$ is not on 4-point line, there exists a colinearly balanced vertical 3separation $\left(X_{i},\left\{b_{i}\right\}, Y_{i}\right)$ of $M$.

Proof. Suppose $M$ is not colinearly entwined and $M$ is not the union of a 4-point line and 4-point coline. Note that $b \notin \Gamma$ for any $b \in B-K$ and any 4-point coline $\Gamma$ of $M$ by Lemma 3.2.3.

5.1.9.1. If $\Gamma_{1}, \Gamma_{2}$ are two distinct 4-point colines of $M$ then $\Gamma_{1} \cap \Gamma_{2}=\emptyset$.

Proof. By Lemma 5.1.6 and the fact that $M$ is not colinearly entwined, $\mid \Gamma_{1} \cap$ $\Gamma_{2} \mid \neq 1$. If $\left|\Gamma_{1} \cap \Gamma_{2}\right| \geq 2$, then $r^{*}\left(\Gamma_{1} \cap \Gamma_{2}\right) \geq 2$, and by Lemma 2.5.7, either $\Gamma_{1}=\Gamma_{2}$; which is not the case, or $\Gamma_{1} \cup \Gamma_{2}$ is a coline of cardinality greater than 4; which contradicts Lemma 5.1.5. So $\Gamma_{1} \cap \Gamma_{2}=\emptyset$.

Suppose $b \in B-K$. Let $(X,\{b\}, Y)$ be a balanced vertical 3-separation of $M$ with $Y \cup\{b\}$ closed. If $M$ does not contain any 4-point colines, then any balanced vertical 3-separation of $M$ is immediately colinearly balanced, and the theorem follows. So assume that $\Gamma_{1}$ is a 4-point coline of $M$. We shall construct a suitable colinearly balanced vertical 3-separation. The construction is a case analysis dependant on the size of $\Gamma_{1} \cap Y$.

$$
\text { Case (i): }\left|\Gamma_{1} \cap Y\right|=4
$$

Suppose $\Gamma_{1} \subseteq Y$. If $\Gamma_{1}$ is the only 4-point coline of $M$, then $(X,\{b\}, Y)$ is colinearly balanced. So let $\Gamma_{2}$ be second 4-point coline of $M$. As $(X,\{b\}, Y)$ is balanced, it must be the case that $\left|\Gamma_{2} \cap X\right| \geq 2$ and $\Gamma_{2} \cap Y \cap K=$ $\emptyset$. By Lemma 3.2.4 and 5.1.9.1, $r\left(Y-\Gamma_{2}\right) \geq 4$. Note that $\Gamma_{2} \subseteq \operatorname{cl}^{*}(X)$. Applying Lemma 3.4.3, and noting that $M$ contains at most two distinct 4-point colines, it follows that $\left(X \cup \Gamma_{2},\{b\}, Y-\Gamma_{2}\right)$ is a colinearly balanced vertical 3-separation of $M$.

Case (ii): $\left|\Gamma_{1} \cap Y\right|=3$

Suppose that $\left|\Gamma_{1} \cap Y\right|=3$ with $\Gamma_{1} \cap X=\left\{z_{1}\right\}$. Then, as $b$ does not lie on a 4-point line, $\left(X-\left\{z_{1}\right\},\{b\}, Y \cup\left\{z_{1}\right\}\right)$ is a vertical 3-separation of $M$ by Lemma 5.1.7. If this vertical 3 -separation is not balanced, then by Lemma 4.1.2, $X-\left\{z_{1}\right\}$ contains a triad $\Lambda$ and there exists some $r \in$ 
$Y \cup\left\{z_{1}\right\}$ such that $\Lambda \cup\{r\}$ is a 4-point coline. By 5.1.9.1, $r \notin \Gamma_{1}$. As $r \in \operatorname{cl}^{*}\left(X-\left\{z_{1}\right\}\right)$, an application of Lemma 3.4.3 yields a colinearly balanced vertical 3-separation $\left((X \cup\{r\})-\left\{z_{1}\right\},\{b\},\left(Y \cup\left\{z_{1}\right\}\right)-\{r\}\right)$ of $M$.

So assume that $\left(X-\left\{z_{1}\right\},\{b\}, Y \cup\left\{z_{1}\right\}\right)$ is balanced. If $\Gamma_{1}$ is the only 4-point line of $M$, then $\left(X-\left\{z_{1}\right\},\{b\}, Y \cup\left\{z_{1}\right\}\right)$ is colinearly balanced. So assume that $M$ contains another 4-point coline $\Gamma_{2}$. Then $\left|\left(X-\left\{z_{1}\right\}\right) \cap \Gamma_{2}\right| \geq 2$ because $K-\Gamma_{1} \subset X \cap \Gamma_{2}$. So $\Gamma_{2}-\left(X-\left\{z_{1}\right\}\right) \subset \operatorname{cl}^{*}\left(X-\left\{z_{1}\right\}\right)$. By 5.1.9.1 and Lemma 3.2.4, $r\left(\left(Y \cup\left\{z_{1}\right\}\right)-\Gamma_{2}\right) \geq 4$, hence, by an application of Lemma 3.4.3, we conclude that $\left(\left(X-\left\{z_{1}\right\}\right) \cup \Gamma_{2},\{b\},\left(Y \cup\left\{z_{1}\right\}\right)-\Gamma_{2}\right)$ is a colinearly balanced vertical 3-separation of $M$.

\section{Case (iii): $\left|\Gamma_{1} \cap Y\right|=2$}

Supose $\Gamma_{1} \cap X=\left\{z_{1}, z_{2}\right\}$. Then $\left(X-\left\{z_{1}\right\},\{b\}, Y \cup\left\{z_{1}\right\}\right)$ is a vertical 3-separation of $M$ by Lemma 5.1.7. Now $z_{2} \in \operatorname{cl}^{*}\left(Y \cup\left\{b, z_{1}\right\}\right)$, so $z_{2} \notin \operatorname{cl}\left(Y \cup\left\{b, z_{1}\right\}\right)$ by Lemma 3.3.7. Therefore, by Lemma 3.3.6, $\left(X-\operatorname{cl}\left(Y \cup\left\{z_{1}\right\}\right),\{b\}, \operatorname{cl}\left(Y \cup\left\{z_{1}\right\}\right)-\{b\}\right)$ is a vertical 3 separation of $M$ with $z_{2} \in X-\operatorname{cl}\left(Y \cup\left\{z_{1}\right\}\right)$. If this vertical 3-separation is not balanced, then by Theorem 4.1.2, $X-\operatorname{cl}\left(Y \cup\left\{z_{1}\right\}\right)$ is a triad contained in a 4-point coline. This contradicts 5.1.9.1. So $\left(X-\operatorname{cl}\left(Y \cup\left\{z_{1}\right\}\right),\{b\}, \operatorname{cl}\left(Y \cup\left\{z_{1}\right\}\right)-\{b\}\right)$ must be balanced. Applying Lemma 5.1 .7 once more, $\left(X-\left(\operatorname{cl}\left(Y \cup\left\{z_{1}\right\}\right) \cup\right.\right.$ $\left.\left.\left\{z_{2}\right\}\right),\{b\}, \operatorname{cl}\left(Y \cup\left\{z_{1}\right\}\right) \cup\left\{z_{2}\right\}\right)$ is a vertical 3-separation of $M$.

If this vertical 3-separation isn't balanced, then by Theorem 4.1.2, $X-$ $\left(\operatorname{cl}\left(Y \cup\left\{z_{1}\right\}\right) \cup\left\{z_{2}\right\}\right)$ contains a triad $\Lambda$ and there exists some $r \in \operatorname{cl}\left(Y \cup\left\{z_{1}\right\}\right) \cup$ $\left\{z_{2}\right\}$ such that $\Lambda \cup\{r\}$ is a 4-point coline. By 5.1.9.1, $r \notin \Gamma_{1}$. Therefore, $\left(\left(X-\left(\operatorname{cl}\left(Y \cup\left\{z_{1}\right\}\right) \cup\left\{z_{2}\right\}\right)\right) \cup\{r\},\{b\},\left(\operatorname{cl}\left(Y \cup\left\{z_{1}\right\}\right) \cup\left\{z_{2}\right\}\right)-\{r\}\right)$ is a colinearly balanced vertical 3-separation of $M$ by Lemmas 3.4.3 and 3.2.4. So assume that $\left(X-\left(\operatorname{cl}\left(Y \cup\left\{z_{1}\right\}\right) \cup\left\{z_{2}\right\}\right),\{b\}, \operatorname{cl}\left(Y \cup\left\{z_{1}\right\}\right) \cup\left\{z_{2}\right\}\right)$ is balanced. If $\Gamma_{1}$ is the only 4-point coline of $M$, we then have our colinearly balanced vertical 3-separation. So assume that $\Gamma_{2}$ is some other 4-point coline of $M$. It must be that $\left|\Gamma_{2} \cap K \cap\left(X-\left(\operatorname{cl}\left(Y \cup\left\{z_{1}\right\}\right) \cup\left\{z_{2}\right\}\right)\right)\right|=2$. Now by 5.1.9.1 together with Lemmas 3.4.3 and 3.2.4, $\left(\left(X-\left(\operatorname{cl}\left(Y \cup\left\{z_{1}\right\}\right) \cup\left\{z_{2}\right\}\right)\right) \cup \Gamma_{2},\{b\},(\operatorname{cl}(Y \cup\right.$ $\left.\left.\left.\left\{z_{1}\right\}\right) \cup\left\{z_{2}\right\}\right)-\Gamma_{2}\right)$ is a colinearly balanced vertical 3-separation of $M$.

Case (iv): $\left|\Gamma_{1} \cap Y\right|=1$ 
Suppose $\Gamma_{1} \cap Y=\left\{z_{1}\right\}$. Then $z_{1} \in \mathrm{cl}^{*}(X)$, so that $z_{1} \notin \operatorname{cl}(X)$ by Lemma 3.3.7. Hence $(\operatorname{cl}(X)-\{b\},\{b\}, Y-\operatorname{cl}(X))$ is a vertical 3-separation with $z_{1} \in(Y-\operatorname{cl}(X))$ by Lemma 3.3.6. If $|(Y-\operatorname{cl}(X)) \cap K|=1$, then $Y-\operatorname{cl}(X)$ is a triad which is contained in some 4-point coline by Theorem 4.1.2. But this contradicts 5.1.9.1. So it must be that $|(Y-\operatorname{cl}(X)) \cap K|=2$ and the vertical 3-separation $(\operatorname{cl}(X)-\{b\},\{b\}, Y-\operatorname{cl}(X))$ is balanced. Now by Lemma 5.1.7, $\left((\operatorname{cl}(X)-\{b\}) \cup\left\{z_{1}\right\},\{b\}, Y-\left(\operatorname{cl}(X) \cup\left\{z_{1}\right\}\right)\right)$ is a vertical 3-separation of $M$. If it isn't balanced, then, by Lemma 4.1.2, $Y-\left(\operatorname{cl}(X) \cup\left\{z_{1}\right\}\right)$ contains a triad $\Lambda$ and there exists some $r \in \operatorname{cl}(X)-\{b\}$ such that $\Lambda \cup\{r\}$ is a 4-point coline. Applying Theorem 3.4.3 with 5.1.9.1 and Lemma 3.2.4, we see that $\left((\operatorname{cl}(X)-\{b, r\}) \cup\left\{z_{1}\right\},\{b\},\left(Y-\left(\operatorname{cl}(X) \cup\left\{z_{1}\right\}\right)\right) \cup\{r\}\right)$ is a colinearly balanced vertical 3-separation of $M$.

So assume that $\left((\operatorname{cl}(X)-\{b\}) \cup\left\{z_{1}\right\},\{b\}, Y-\left(\operatorname{cl}(X) \cup\left\{z_{1}\right\}\right)\right)$ is balanced. If it is still not colinearly balanced, then there must exist another 4-point coline $\Gamma_{2}$ in $M$. Then $\left|\Gamma_{2} \cap K \cap\left(Y-\left(\operatorname{cl}(X) \cup\left\{z_{1}\right\}\right)\right)\right|=2$, so $\Gamma_{2} \in \operatorname{cl}^{*}(Y-(\operatorname{cl}(X) \cup$ $\left.\left.\left\{z_{1}\right\}\right)\right)$. By 5.1.9.1, and Lemmas 3.2.4 and 3.4.3, we again find a colinearly balanced vertical 3-separation $\left(\left((\operatorname{cl}(X)-\{b\}) \cup\left\{z_{1}\right\}\right)-\Gamma_{2},\{b\},(Y-(\operatorname{cl}(X) \cup\right.$ $\left.\left.\left.\left\{z_{1}\right\}\right)\right) \cup \Gamma_{2}\right)$ of $M$.

$$
\text { Case (v): }\left|\Gamma_{1} \cap Y\right|=0
$$

Suppose $\Gamma_{1} \cap Y=\emptyset$. Then $\Gamma_{1} \subset X$. If $(X,\{b\}, Y)$ isn't colinearly balanced, then $M$ contains some other 4-point coline $\Gamma_{2}$. Then $\left|\Gamma_{2} \cap Y \cap K\right|=$ 2. Applying 5.1.9.1, Lemma 3.2.4 and Lemma 3.4.3 once more, we conclude that $\left(X-\Gamma_{2},\{b\}, Y \cup \Gamma_{2}\right)$ is a colinearly balanced vertical 3-separation of $M$.

Corollary 5.1.10. Let $M$ be a 3-connected matroid with no 4-element fans, and let $B$ be a basis of $M$. Let $K$ denote the set of elements which are removable with respect to $B$, and suppose $|K|=4$. If $(X,\{b\}, Y)$ is a colinearly balanced vertical 3-separation of $M$, then $(\operatorname{cl}(X)-\{b\},\{b\}, Y-\operatorname{cl}(X))$ is also a colinearly balanced vertical 3-separation of $M$.

Proof. Let $(X,\{b\}, Y)$ be a colinearly balanced vertical 3-separation of $M$. By Lemma 3.3.6, $(\operatorname{cl}(X)-\{b\},\{b\}, Y-\operatorname{cl}(X))$ is a vertical 3-separation of $M$ with $(\operatorname{cl}(X)-\{b\}) \cup\{b\}=\operatorname{cl}(X)$ closed. If $(\operatorname{cl}(X)-\{b\},\{b\}, Y-\operatorname{cl}(X))$ is not balanced, then by Theorem 4.1.2, $Y-\operatorname{cl}(X)$ is a triad and there exists some $r \in \operatorname{cl}(X)-\{b\}$ such that $(Y-\operatorname{cl}(X)) \cup\{r\}$ is a 4 -point coline. But 
then $r \in \mathrm{cl}^{*}(Y-\operatorname{cl}(X))$, so that $r \notin \operatorname{cl}(\operatorname{cl}(X)-\{r\})$. This now implies that $r(\operatorname{cl}(X))>r(\operatorname{cl}(X)-\{r\})$, which contradicts the fact that $r \in \operatorname{cl}(X)$. So $(\operatorname{cl}(X)-\{b\},\{b\}, Y-\operatorname{cl}(X))$ must be balanced. Suppose $Y$ contains a 4-point coline $\Gamma$. Then $|(Y-\operatorname{cl}(X)) \cap \Gamma \cap K|=2$. As $\gamma_{i} \in \operatorname{cl}^{*}\left(\Gamma-\gamma_{i}\right)$ for all $\gamma_{i} \in \Gamma$, it follows that $\gamma_{i} \notin \operatorname{cl}(X \cup\{b\})=\operatorname{cl}(X)$ for all $\gamma_{i} \in \Gamma$ by Lemma 2.5.4. Thus $\Gamma \subseteq Y-\operatorname{cl}(X)$ and $(\operatorname{cl}(X)-\{b\},\{b\}, Y-\operatorname{cl}(X))$ is colinearly balanced.

Corollary 5.1.11. Let $M$ be a 3-connected matroid with no 4-element fans, and let $B$ be a basis of $M$. Let $K$ denote the set of elements which are removable with respect to $B$. Suppose $|K|=4$ and there exists some $b \in$ $B-K$. If $M^{*}$ is not colinearly entwined and $M$ is not the union of a 4-point line and a 4-point coline, then for each $c_{i} \in(E(M)-B)-K$ such that $c_{i}$ is not on 4-point coline, there exists a linearly balanced cyclic 3-separation $\left(S_{i},\left\{c_{i}\right\}, T_{i}\right)$ of $M$.

Proof. Note that a cyclic 3-separation $(S,\{c\}, T)$ of $M$ is linearly balanced if and only if $(S,\{c\}, T)$ is a colinearly balanced vertical 3-separation of $M^{*}$. The corollary then follows by noting Lemmas 3.5.5 and 3.5.3 and dualising Proposition 5.1.9.

Corollary 5.1.12. Let $M$ be a 3-connected matroid with no 4-element fans, and let $B$ be a basis of $M$. Let $K$ denote the set of elements which are removable with respect to $B$, and suppose $|K|=4$. If $(S,\{c\}, T)$ is a linearly balanced cyclic 3-separation of $M$, then $\left(\mathrm{cl}^{*}(S)-\{c\},\{c\}, T-\mathrm{cl}^{*}(S)\right)$ is also a linearly balanced cyclic 3-separation of $M$.

Proof. This follows by dualising Corollary 5.1.10.

\subsection{Arranging Removable Elements}

Theorem 5.2.1. Let $M$ be a 3-connected matroid with no 4-element fans, where $M$ is not the union of a 4-point line and a 4-point coline. Let $B$ be a basis of $M$, and let $K$ denote the set of elements which are removable with respect to $B$. Suppose $|K|=4$. Then there exists a set $\left\{\alpha_{1}, \alpha_{2}\right\} \subset K$ such that for every $b_{i} \in B-K$, there exists a balanced vertical 3-separation $\left(X_{i},\left\{b_{i}\right\}, Y_{i}\right)$ with $\left\{\alpha_{1}, \alpha_{2}\right\} \subset X_{i}$. Moreover, if $M$ contains a 4-point line, then $\left\{\alpha_{1}, \alpha_{2}\right\} \subset(E(M)-B) \cap L$ where $L$ is a 4-point line of $M$. 
Proof. If $M$ is colinearly entwined, then the theorem follows immediately from Theorem 5.1.8. So assume that $M$ is not colinearly entwined. Our argument is divided into three separate cases.

Case (i): $(E(M)-B)-K=\emptyset$.

Suppose that $(E(M)-B)-K=\emptyset$, and $b_{1} \in(B-K)$. Let $\left(X,\left\{b_{1}\right\}, Y\right)$ be a balanced vertical 3-separation of $M$ and let $\left\{\alpha_{1}, \alpha_{2}\right\}=X \cap K$. We show that for all other $b_{i} \in B-K$, there exists a balanced vertical 3-separation $\left(X_{i},\left\{b_{i}\right\}, Y_{i}\right)$ with $\left\{\alpha_{1}, \alpha_{2}\right\} \subset X_{i}$.

Take some $b_{2} \in B-K$, and assume that there does not exist any balanced vertical 3-separation $\left(S,\left\{b_{2}\right\}, T\right)$ with $\left\{\alpha_{1}, \alpha_{2}\right\} \subset S$. Then $b_{1} \neq b_{2}$. Let $\left(P,\left\{b_{2}\right\}, Q\right)$ be a balanced vertical 3 -separation of $M$. Without loss of generality, we may assume that $b_{1} \in Q$ and $b_{2} \in Y$.

5.2.1.1. For all $y \in(Y \cap K) \cup\left\{b_{2}\right\}, y \notin \operatorname{cl}(X)$.

Proof. If $b_{2} \in \operatorname{cl}(X)$, then, by Lemma 3.2.8, ( $\left.X \cup\left\{b_{1}\right\},\left\{b_{2}\right\}, Y-\left\{b_{2}\right\}\right)$ is a vertical 3-separation that contradicts our assumption. So $b_{2} \notin \operatorname{cl}(X)$. Suppose $z \in Y \cap K \cap \operatorname{cl}(X)$. Then by Lemma 3.3.6, $\left(\operatorname{cl}(X)-\left\{b_{1}\right\},\left\{b_{1}\right\}, Y-\right.$ $\operatorname{cl}(X))$ is a vertical 3-separation of $M$ with $|(Y-\operatorname{cl}(X)) \cap K| \leq 1$. Now by Theorem 4.1.2, $|(Y-\mathrm{cl}(X)) \cap K|=1$ and $Y-\operatorname{cl}(X)$ is a triad which contains two elements of $(E(M)-B)-K$. But $(E(M)-B)-K=\emptyset$. Therefore $Y \cap K \cap \operatorname{cl}(X)=\emptyset$.

By Lemma 3.3.6, we may assume; relabelling as necessary, that $\left(X,\left\{b_{1}\right\}, Y\right)$ is a vertical 3-separation such that $X \cup\left\{b_{1}\right\}$ is closed. Moreover, by 5.2.1.1, we maintain the property that $\left(X,\left\{b_{1}\right\}, Y\right)$ is balanced with $b_{2} \in Y$. By assumption, $\left\{\alpha_{1}, \alpha_{2}\right\} \not \subset P$ and $\left\{\alpha_{1}, \alpha_{2}\right\} \not \subset Q$. Without loss of generality, $\alpha_{1} \in X \cap P$ and $\alpha_{2} \in X \cap Q$. Also, $|Y \cap P \cap K|=|Y \cap Q \cap K|=1$ because $\left(X,\left\{b_{1}\right\}, Y\right)$ and $\left(P,\left\{b_{2}\right\}, Q\right)$ are balanced. So each of the intersections $X \cap P, X \cap Q, Y \cap P$ and $Y \cap Q$ are non-empty and each contains exactly one element of $K$.

5.2.1.2. Either $|Y \cap P|=1$ or $(Y \cap P) \cup\left\{b_{2}\right\}$ is a triangle.

Proof. Assume that $|Y \cap P| \geq 2$. As $\lambda(Y)=\lambda(P)=2$ and $|E-(Y \cup P)|=$ $\left|(X \cap Q) \cup\left\{b_{1}\right\}\right| \geq 2$, it follows by uncrossing that $\lambda(Y \cap P)=2$. Similarly, 
$\lambda\left((Y \cap P) \cup\left\{b_{2}\right\}\right)=2$. By Lemmas 3.2.7 and 2.5.4, $b_{2} \in \operatorname{cl}(Y \cap P)$. If $r(Y \cap P) \geq 3$, then $\left(Y \cap P,\left\{b_{2}\right\}, X \cup Q\right)$ is a vertical 3-separation of $M$. But then by Theorem 4.1.2, $Y \cap P$ contains a triad $\Lambda$ which contains elements of $E(M)-B$ which are not removable with respect to $B$; contrary to our assumption. So $r(Y \cap P)=2$. If $|Y \cap P| \geq 3$, then $(Y \cap P) \cup\left\{b_{2}\right\}$ contains a 4-point line $L$. But then $|L \cap Y \cap P \cap(E(M)-B)| \geq 2$ by Lemma 2.3.3, and so $|Y \cap P \cap K| \geq 2$ by Lemma 3.2.2, which is a contradiction. Therefore, if $|Y \cap P| \geq 2$, then $(Y \cap P) \cup\left\{b_{2}\right\}$ is a triangle. Otherwise, $|Y \cap P|=1$.

5.2.1.3. $|X \cap P| \geq 2$.

Proof. Suppose $|X \cap P|=1$. Then $(Y \cap P) \cup\left\{b_{2}\right\}$ is a triangle by 5.2.1.2 and the fact that $r(P) \geq 3$. But then $P$ is a triad, so that $P \cup\left\{b_{2}\right\}$ is a contradictory 4-element fan.

5.2.1.4. $(Y \cap Q) \cup\left\{b_{1}, b_{2}\right\}$ is a triangle.

Proof. Noting that $\lambda(Y)=\lambda(Q)=2$ and $|E-(Y \cup Q)|=|X \cap P| \geq 2$ by 5.2 .1 .3 , it follows by uncrossing that $\lambda(Y \cap Q) \leq 2$. Similarly we obtain $\lambda\left((Y \cap Q) \cup\left\{b_{2}\right\}\right)=\lambda\left((Y \cap Q) \cup\left\{b_{1}, b_{2}\right\}\right)=2$. Now by Lemmas 3.2.7 and 2.5.4, $b_{1} \in \operatorname{cl}\left((Y \cap Q) \cup\left\{b_{2}\right\}\right)$. As $M$ is 3-connected, all that remains is to show that $|Y \cap Q|=1$. Suppose $|Y \cap Q| \geq 2$. Then $\lambda(Y \cap Q)=2$ and it follows that $b_{2} \in \operatorname{cl}(Y \cap Q)$. If $r(Y \cap Q) \geq 3$, then $\left(Y \cap Q,\left\{b_{2}\right\}, X \cup P \cup\left\{b_{1}\right\}\right)$ is a vertical 3 -separation of $M$. But then Theorem 4.1.2 implies that $Y \cap Q$ contains a triad $\Lambda$ that contains two elements of $(E(M)-B)-K$, which is absurd. So $r(Y \cap Q)=2$. But then $r\left((Y \cap Q) \cup\left\{b_{1}, b_{2}\right\}\right)=2$ and $(Y \cap Q) \cup\left\{b_{1}, b_{2}\right\}$ contains a 4-point line $L$. By Lemma 2.3.3, $Y \cap Q \subseteq E(M)-B$, and, as $|Y \cap Q \cap L| \geq 2$, we get $|Y \cap Q \cap K| \geq 2$ by Lemma 3.2.2. This is a contradiction. We conclude that $|Y \cap Q|=1$ and that $(Y \cap Q) \cup\left\{b_{1}, b_{2}\right\}$ is a triangle.

Now suppose that $(Y \cap P) \cup\left\{b_{2}\right\}$ is a triangle. Note that $Y$ is a rank 3 cocircuit of $M$. Let $Y \cap P=\left\{z_{1}, z_{2}\right\}$. Then $r\left(Y-\left\{z_{1}\right\}\right)=r\left(Y-\left\{z_{2}\right\}\right)=3$. Therefore, both $\operatorname{co}\left(M \backslash z_{1}\right)$ and $\operatorname{co}\left(M \backslash z_{2}\right)$ are 3-connected by Theorem 3.4.4. As $|Y \cap P \cap K|=1$, it follows from Lemma 2.3.3 that $|Y \cap P \cap(B-K)|=1$. Without loss of generalization, $z_{1} \in B-K$. Let $Y \cap Q=\{w\}$. Now, by Lemma 2.6.5, each of $b_{1}, b_{2}$ and $w$ are contained in a distinct parallel class of $M / z_{1}$. But this, together with the fact that $z_{1} \notin K$ contradicts Lemma 3.4.1. 
So $(Y \cap P) \cup\left\{b_{2}\right\}$ is not a triangle, and by 5.2.1.2, it must be that $|Y \cap P|=1$. But then $Y$ is a triad and so $Y \cup\left\{b_{1}\right\}$ is a contradictory 4-element fan in $M$. We conclude that either $\left\{\alpha_{1}, \alpha_{2}\right\} \subset P$ or $\left\{\alpha_{1}, \alpha_{2}\right\} \subset Q$ as required.

We now assume for the remaining two cases that $(E(M)-B)-K \neq \emptyset$.

Case (ii): $M$ contains a 4-point line.

Let $L$ be a 4-point line of $M$. Then $|L \cap K \cap(E(M)-B)| \geq 2$ by Lemmas 3.2.2 and 2.3.3. Choose distinct $\alpha_{1}, \alpha_{2} \in L \cap K \cap(E(M)-B)$. We show that for each $b_{i} \in B-K$, there exists a balanced vertical 3-separation $\left(X_{i},\left\{b_{i}\right\}, Y_{i}\right)$ such that $\left\{\alpha_{1}, \alpha_{2}\right\} \subset X_{i}$.

Suppose $b \in B-K$. Let $(X,\{b\}, Y)$ be a vertical 3-separation of $M$. Without loss of generality, we may assume that $|X \cap L| \geq 2$. By Lemma 3.3.6, $(\operatorname{cl}(X)-\{b\},\{b\}, Y-\operatorname{cl}(X))$ is a vertical 3-separation of $M$ with $\left\{\alpha_{1}, \alpha_{2}\right\} \subset$ $L \subset \operatorname{cl}(X)-\{b\}$. If $(\operatorname{cl}(X)-\{b\},\{b\}, Y-\operatorname{cl}(X))$ is balanced, we're done. So suppose $(\operatorname{cl}(X)-\{b\},\{b\}, Y-\operatorname{cl}(X))$ is not balanced. Then $\mid(Y-\operatorname{cl}(X)) \cap$ $K \mid=1$, and by Theorem 4.1.2, $Y-\operatorname{cl}(X)$ is a triad and there exists $r \in B \cap K$ such that $(Y-\operatorname{cl}(X)) \cup\{r\}$ is a 4-point coline. So $r \in \mathrm{cl}^{*}(Y-\operatorname{cl}(X))$ and, by Lemma 2.5.4, $r \notin L$. If $r(\operatorname{cl}(X)-\{b, r\})=2$, then it must be that $\operatorname{cl}(X)-\{r\}=L$. But then $M$ is the union of a 4-point line and a 4-point coline, which is contrary to our assumption. So $r(\operatorname{cl}(X)-\{b, r\}) \geq 3$, and applying Lemma 3.4.3, we conclude that $(\operatorname{cl}(X)-\{b, r\},\{b\},(Y-\operatorname{cl}(X)) \cup\{r\})$ is a balanced vertical 3-separation with $\left\{\alpha_{1}, \alpha_{2}\right\} \subset \operatorname{cl}(X)-\{b, r\}$ as required.

The argument above applies to any $b_{i} \in B-K$, so that if $M$ contains a 4-point line, the theorem holds.

Case (iii): $M$ does not contain a 4-point line.

Now assume that $M$ does not contain any 4-point lines. As $M$ is not colinearly entwined, we deduce from Theorem 5.1.9 that for each $b_{i} \in B-K$, there exists a colinearly balanced vertical 3-separation $\left(X_{i},\left\{b_{i}\right\}, Y_{i}\right)$ of $M$. Choose some $b_{1} \in B-K$ and let $\left(X,\left\{b_{1}\right\}, Y\right)$ be a colinearly balanced vertical 3-separation. Let $X \cap K=\left\{\alpha_{1}, \alpha_{2}\right\}$. We show that for any $b_{i} \in B-K$, there exists a balanced vertical 3 -separation $\left(X_{i},\left\{b_{i}\right\}, Y_{i}\right)$ with $\left\{\alpha_{1}, \alpha_{2}\right\} \subset X_{i}$.

Suppose $b_{2} \in B-K$. Assume that there does not exist any balanced vertical 3-separation $\left(S,\left\{b_{2}\right\}, T\right)$ with $\left\{\alpha_{1}, \alpha_{2}\right\} \subset S$. Then $b_{1} \neq b_{2}$. Let 
$\left(P,\left\{b_{2}\right\}, Q\right)$ be a colinearly balanced vertical 3-separation of $M$. Without loss of generality, we may assume that $b_{1} \in Q$ and $b_{2} \in Y$. If $b_{2} \in \operatorname{cl}(X)$, then by Lemma 3.2.8, $\left(X \cup\left\{b_{1}\right\},\left\{b_{2}\right\}, Y-\left\{b_{2}\right\}\right)$ is a balanced vertical 3separation of $M$ that contradicts our assumption. So $b_{2} \notin \operatorname{cl}(X)$. Now by Corollary 5.1.10, we may assume; relabelling as appropriate, that $X \cup\left\{b_{1}\right\}$ is closed with $b_{2} \in Y$. We may also assume by Corollary 5.1.10 that $Q \cup\left\{b_{2}\right\}$ is closed.

By assumption $\left\{\alpha_{1}, \alpha_{2}\right\} \not \subset P$ and $\left\{\alpha_{1}, \alpha_{2}\right\} \not \subset Q$. Without loss of generality, $\alpha_{1} \in X \cap P$ and $\alpha_{2} \in X \cap Q$. As $\left(X,\left\{b_{1}\right\}, Y\right)$ and $\left(P,\left\{b_{2}\right\}, Q\right)$ are balanced, it must also be that $|Y \cap P \cap K|=|Y \cap Q \cap K|=1$. So each of the intersections $X \cap P, X \cap Q, Y \cap P$, and $Y \cap Q$ are non-empty and each contains exactly one element of $K$.

5.2.1.5. Either $|Y \cap P|=1$ or $(Y \cap P) \cup\left\{b_{2}\right\}$ is a triangle.

Proof. Assume that $|Y \cap P| \geq 2$. As $\lambda(Y)=\lambda(P)=2$ and $|E-(Y \cup P)|=$ $\left|(X \cap Q) \cup\left\{b_{1}\right\}\right| \geq 2$, it follows by uncrossing that $\lambda(Y \cap P)=2$. Similarly, $\lambda\left((Y \cap P) \cup\left\{b_{2}\right\}\right)=2$. By Lemmas 3.2.7 and 2.5.4, $b_{2} \in \operatorname{cl}(Y \cap P)$. If $r(Y \cap P) \geq 3$, then $\left(Y \cap P,\left\{b_{2}\right\}, X \cup Q\right)$ is a vertical 3-separation of $M$. But then by Theorem 4.1.2, $Y \cap P$ contains a triad $\Lambda$ and there exists some $r \in X \cup Q$ such that $\Lambda \cup\{r\}$ is a 4-point coline. But $\left(X,\left\{b_{1}\right\}, Y\right)$ and $\left(P,\left\{b_{2}\right\}, Q\right)$ are colinearly balanced so it must be that $r \in Y \cap P$, which is absurd. Hence $r(Y \cap P) \leq 2$. If $|Y \cap P| \geq 3$, then $(Y \cap P) \cup\left\{b_{2}\right\}$ contains a 4-point line. But $M$ does not contain any such lines by assumption. So $|Y \cap P| \geq 2 \Longrightarrow(Y \cap P) \cup\left\{b_{2}\right\}$ is a triangle. Otherwise, $|Y \cap P|=1$.

5.2.1.6. $\lambda(Y \cap Q) \leq 2$, and $\lambda\left((Y \cap Q) \cup\left\{b_{2}\right\}\right)=\lambda\left((Y \cap Q) \cup\left\{b_{1}, b_{2}\right\}\right)=2$.

Proof. Consider $|X \cap P|$. If $|X \cap P|=1$, then $(Y \cap P) \cup\left\{b_{2}\right\}$ is a triangle by 5.2.1.5 and the fact that $r(P) \geq 3$. Recall that $Q \cup\left\{b_{2}\right\}$ is closed. But now $P$ is a triad, and $Y \cup\left\{b_{2}\right\}$ is a contradictory 4-element fan in $M$. Therefore $|X \cap P| \geq 2$. Thus $|E-(Y \cup Q)| \geq 2$, and by uncrossing we get that $\lambda(Y \cap Q) \leq$ 2. Similarly, $\left|E-\left(Y \cup\left(Q \cup\left\{b_{2}\right\}\right)\right)\right| \geq 2$ and $\left|E-\left(\left(Y \cup\left\{b_{1}\right\}\right) \cup\left(Q \cup\left\{b_{2}\right\}\right)\right)\right| \geq 2$, so that by uncrossing, $\lambda\left((Y \cap Q) \cup\left\{b_{2}\right\}\right) \leq 2$ and $\lambda\left((Y \cap Q) \cup\left\{b_{1}, b_{2}\right\}\right) \leq 2$. As $M$ is 3-connected, we obtain $\lambda\left((Y \cap Q) \cup\left\{b_{2}\right\}\right)=\lambda\left((Y \cap Q) \cup\left\{b_{1}, b_{2}\right\}\right)=2$.

5.2.1.7. $(Y \cap Q) \cup\left\{b_{1}, b_{2}\right\}$ is a triangle. 
Proof. Suppose $|Y \cap Q| \geq 2$ so that $\lambda(Y \cap Q)=2$. It then follows from 5.2.1.6 and Lemmas 3.2.7 and 2.5.4 that $\left\{b_{1}, b_{2}\right\} \subset \operatorname{cl}(Y \cap Q)$. If $r(Y \cap Q) \geq 3$, then $\left(Y \cap Q,\left\{b_{2}\right\}, X \cup P \cup\left\{b_{1}\right\}\right)$ is a vertical 3-separation of $M$. But $|Y \cap Q \cap K|=1$, so that by Theorem 4.1.2, $Y \cap Q$ contains a triad $\Lambda$ which is contained in a 4point line $\Gamma \not \subset Y \cap Q$. But this is absurd as both $\left(X,\left\{b_{1}\right\}, Y\right)$ and $\left(P,\left\{b_{2}\right\}, Q\right)$ are colinearly balanced. So $r(Y \cap Q)=2$. But then $(Y \cap Q) \cup\left\{b_{1}, b_{2}\right\}$ contains a 4-point line which cannot exist. Thus $|Y \cap Q|=1$. By 5.2.1.6, $\lambda\left((Y \cap Q) \cup\left\{b_{2}\right\}\right)=\lambda\left((Y \cap Q) \cup\left\{b_{1}, b_{2}\right\}\right)=2$, and Lemmas 3.2.7 and 2.5.4 imply that $b_{1} \in \operatorname{cl}\left((Y \cap Q) \cup\left\{b_{2}\right\}\right)$. As $M$ is 3-connected, $(Y \cap Q) \cup\left\{b_{1}, b_{2}\right\}$ must be a triangle.

Recall from 5.2.1.5 that either $|Y \cap P|=1$ or $(Y \cap P) \cup\left\{b_{2}\right\}$ is a triangle. Suppose $(Y \cap P) \cup\left\{b_{2}\right\}$ is a triangle. Let $Y \cap P=\left\{z_{1}, z_{2}\right\}$ and $Y \cap Q=\{w\}$. Note that $Y$ is a rank 3 cocircuit of $M$. Now $r\left(Y-\left\{z_{1}\right\}\right)=r\left(Y-\left\{z_{2}\right\}\right)=3$. Therefore by Theorem 3.4.4, both $\operatorname{co}\left(M \backslash z_{1}\right)$ and $\operatorname{co}\left(M \backslash z_{2}\right)$ are 3-connected. But $|Y \cap P \cap K|=1$ and $\left\{z_{1}, z_{2}, b_{2}\right\}$ is a triangle, so $Y \cap P$ must contain exactly one basis element. Without loss of generalization, $z_{1} \in B$. But then, by Lemma 2.6.5, $b_{1}, b_{2}$ and $w$ are each contained in a distinct parallel class of $M / z_{1}$. Therefore by Lemma 3.4.1, $\operatorname{si}\left(M / z_{1}\right)$ is 3-connected in addition to $\operatorname{co}\left(M \backslash z_{2}\right)$ being 3 -connected. This contradicts the fact that $Y \cap P$ contains exactly one element which is removable with respect to $B$.

The final remaining possibility is that $|Y \cap P|=1$. But then $Y$ is a triad, whereas $(Y \cap Q) \cup\left\{b_{1}, b_{2}\right\}$ is a triangle by 5.2.1.7. This gives a contradictory 4-element fan $Y \cup\left\{b_{1}\right\}$ in $M$. We conclude that $\left\{\alpha_{1}, \alpha_{2}\right\} \subset P$ or $\left\{\alpha_{1}, \alpha_{2}\right\} \subset Q$ as required.

Corollary 5.2.2. $M$ be a 3-connected matroid with no 4-element fans, where $M$ is not the union of a 4-point line and a 4-point coline. Let $B$ be a basis of $M$, and let $K$ denote the set of elements which are removable with respect to B. Suppose $|K|=4$. Then there exists a set $\left\{\beta_{1}, \beta_{2}\right\} \subset K$ such that for every $c_{i} \in(E(M)-B)-K$, there exists a balanced cyclic 3-separation $\left(S_{i},\left\{c_{i}\right\}, T_{i}\right)$ with $\left\{\beta_{1}, \beta_{2}\right\} \subset S_{i}$. Moreover, if $M$ contains a 4-point coline, then $\left\{\beta_{1}, \beta_{2}\right\} \subset B \cap \Gamma$ where $\Gamma$ is a 4-point coline of $M$.

Proof. Noting that $(X,\{b\}, Y)$ is a balanced vertical 3-separation of a matroid $M$ iff it is a balanced cyclic 3-separation of $M^{*}$, this result follows by dualising Theorem 5.2.1. 
Theorem 5.2.3. Let $M$ be a 3-connected matroid with no 4-element fans, where $M$ is not the union of a 4-point line and a 4-point coline. Let $B$ be a basis of $M$, and let $K$ denote the set of elements which are removable with respect to $B$. Suppose $|K|=4$. Then there exists a set $\left\{k_{1}, k_{2}\right\} \subset K$ such that:

(i) for every $b_{i} \in B-K$, there exists a balanced vertical 3-separation $\left(X_{i},\left\{b_{i}\right\}, Y_{i}\right)$ with $\left\{k_{1}, k_{2}\right\} \subset X_{i}$, and

(ii) for every $c_{i} \in(E(M)-B)-K$, there exists a balanced cyclic 3separation $\left(S_{i},\left\{c_{i}\right\}, T_{i}\right)$ with $\left\{k_{1}, k_{2}\right\} \subset S_{i}$.

Proof. If either $B-K=\emptyset$ or $(E(M)-B)-K=\emptyset$, then this follows immediately from the previous two results. So we can assume that both are non-empty. Our argument is divided into three cases.

Case (i): $M$ contains a 4-point line.

Suppose $M$ contains a 4-point line. Then by Theorem 5.2.1, there exists a 4-point line $L$ with a set $\left\{\alpha_{1}, \alpha_{2}\right\} \subset(E(M)-B) \cap L \cap K$ such that for every $b_{i} \in B-K$ there exists a balanced vertical 3-separation $\left(X_{i},\left\{b_{i}\right\}, Y_{i}\right)$ with $\left\{\alpha_{1}, \alpha_{2}\right\} \subset X_{i}$. Let $\left\{k_{1}, k_{2}\right\}=\left\{\alpha_{1}, \alpha_{2}\right\}$. Then (i) is immediate. We now prove (ii). If $M^{*}$ is colinearly entwined, then (ii) follows directly from the dual of Proposition 5.1.8. So assume that $M^{*}$ is not colinearly entwined. For each $c_{i} \in(E(M)-B)-K$ where $c_{i}$ does not lie on a 4-point coline, there exists a linearly balanced cyclic 3-separation $\left(S_{i},\left\{c_{i}\right\}, T_{i}\right)$ of $M$ by Corollary 5.1.11. It follows that (ii) holds if $M$ does not contain a 4 -point coline. So let $\Gamma$ be a 4-point coline of $M$.

Choose any $c \in(E(M)-B)-K$ and let $(S,\{c\}, T)$ be a balanced cyclic 3-separation of $M$. Without loss of generality, $|T \cap \Gamma| \geq 2$. By Lemma 3.3.5, $\left(S-\mathrm{cl}^{*}(T),\{c\}, \mathrm{cl}^{*}(T)-\{c\}\right)$ is a cyclic 3-separation of $M$ with $\Gamma \subset \mathrm{cl}^{*}(T)$. Suppose $\left(S-\mathrm{cl}^{*}(T),\{c\}, \mathrm{cl}^{*}(T)-\{c\}\right)$ is not balanced. Then by Corollary 4.1.3, $\left|\left(S-\mathrm{cl}^{*}(T)\right) \cap K\right|=1$ and $S-\mathrm{cl}^{*}(T)$ is a triangle which is contained in a 4-point line. This 4-point line must be $L$. Without loss of generality, $\left(\mathrm{cl}^{*}(T)-\{c\}\right) \cap L=\left\{k_{1}\right\}$. Note that $k_{1} \notin \Gamma$. Now $k_{1} \in \mathrm{cl}\left(S-\mathrm{cl}^{*}(T)\right)$, and by Lemma 3.2.8, $\mathrm{cl}^{*}(T)-\left\{k_{1}\right\}$ is an exactly 3-separating set. If $\mathrm{cl}^{*}(T)-\left\{k_{1}\right\}$ does not contain a circuit, then by Lemma 3.2.6, $\mathrm{cl}^{*}(T)-\left\{k_{1}\right\}$ is a coline. As $\Gamma \subseteq \operatorname{cl}^{*}(T)-\left\{k_{1}\right\}$ and $M$ contains no colines of size greater than 
4 by Lemma 5.1.5, it follows that $\mathrm{cl}^{*}(T)-\left\{k_{1}\right\}=\Gamma$. But then $M$ is the union of a 4-point line and a 4-point coline, which is not the case. Therefore $\left(S-\mathrm{cl}^{*}(T),\{c\}, \mathrm{cl}^{*}(T)-\{c\}\right)$ must be balanced, and $\left\{k_{1}, k_{2}\right\} \subset S-\mathrm{cl}^{*}(T)$ as required. This applies to any $c_{i} \in(E(M)-B)-K$ so that we obtain (ii) and conclude that the theorem holds.

Case (ii): $M$ contains a 4-point coline.

Suppose $M$ contains a 4-point coline. Then by Theorem 5.2.2, there exists a 4-point coline $\Gamma$ with a set $\left\{\beta_{1}, \beta_{2}\right\} \subset B \cap \Gamma \cap K$ such that for every $c_{i} \in$ $(E(M)-B)-K$ there exists a balanced cylic 3 -separation $\left(S_{i},\left\{c_{i}\right\}, T_{i}\right)$ with $\left\{\beta_{1}, \beta_{2}\right\} \subset S_{i}$. Let $\left\{k_{1}, k_{2}\right\}=\left\{\beta_{1}, \beta_{2}\right\}$. Then (ii) is immediate. We show (i). If $M$ is colinearly entwined, then (ii) follows directly from Proposition 5.1.8. So assume that $M$ is not colinearly entwined. If $M$ contains a 4-point line, then the theorem holds as in case (i) above. So we can assume that $M$ does not contain any 4-point lines. Then, by Corollary 5.1.9, for every $b_{i} \in$ $B-K$, there exists a colinearly balanced vertical 3-separation $\left(X_{i},\left\{b_{i}\right\}, Y_{i}\right)$ of $M$. Any such 3-separation has $\Gamma \subseteq X_{i}$ or $\Gamma \subseteq Y_{i}$ by construction, so that $\left\{k_{1}, k_{2}\right\} \subset X_{i}$ or $\left\{k_{1}, k_{2}\right\} \subset Y_{i}$ as required. Therefore (i) holds, and the Theorem follows.

Case (iii): $M$ contains no 4-point lines and no 4-point colines.

Suppose that $M$ does not contain any 4-point lines or 4-point colines. By Theorem 5.2.1, there exists a set $\left\{\alpha_{1}, \alpha_{2}\right\} \subset K$ such that for each $b_{i} \in B-K$ there exists a balanced vertical 3-separation $\left(X_{i},\left\{b_{i}\right\}, Y_{i}\right)$ with $\left\{\alpha_{1}, \alpha_{2}\right\} \subset$ $X_{i}$. Let $\left\{k_{1}, k_{2}\right\}=\left\{\alpha_{1}, \alpha_{2}\right\}$. Then (i) holds. We prove (ii). Take some $b \in B-K$ and let $(X,\{b\}, Y)$ be a balanced vertical 3-separation of $M$ with $\left\{k_{1}, k_{2}\right\} \subset X$. We show that for any $c_{i} \in(E(M)-B)-K$, there exists a balanced cyclic 3 -separation $\left(S_{i},\left\{c_{i}\right\}, T_{i}\right)$ with $\left\{k_{1}, k_{2}\right\} \subset S_{i}$.

Suppose $c \in(E(M)-B)-K$. Assume that there does not exist any balanced cyclic 3-separation $(P,\{c\}, Q)$ with $\left\{k_{1}, k_{2}\right\} \subset P$. Let $(S,\{c\}, T)$ be a balanced cyclic 3 -separation of $M$. Without loss of generality, we may assume that $c \in Y$ and $b \in T$

5.2.3.1. For all $y \in(Y \cap K) \cup\{c\}, y \notin \operatorname{cl}(X)$. 
Proof. If $c \in \operatorname{cl}(X)$, then, by Lemma 3.2.8, $(X \cup\{b\},\{c\}, Y-\{c\})$ is a vertical 3-separation of $M$. Therefore $(X \cup\{b\}, Y-\{c\})$ is a 2separation of $M / c$. But $r_{M / c}(X \cup\{b\}) \geq 2$ and $r_{M / c}(Y-\{c\}) \geq 2$, so that $((E(\operatorname{si}(M / c))) \cap(X \cup\{b\}),(E(\operatorname{si}(M / c))) \cap(Y-\{c\}))$ is a 2-separation of $\operatorname{si}(M / c)$. Therefore $\operatorname{si}(M / c)$ is not 3-connected. But $c \in(E(M)-B)-K$, so that $\operatorname{co}(M \backslash c)$ is not 3-connected. This contradicts Lemma 3.4.2. Thus $c \notin$ $\operatorname{cl}(X)$. If $y \in Y \cap K \cap \operatorname{cl}(X)$, then by Lemma 3.3.6, $(\operatorname{cl}(X)-\{b\},\{b\}, Y-\operatorname{cl}(X))$ is a vertical 3-separation of $M$. But then Theorem 4.1.2 implies that $|(Y-\operatorname{cl}(X)) \cap K|=1$ and $Y-\operatorname{cl}(X)$ is contained in a 4-point coline; contrary to our assumption that $M$ contains no such colines. So $Y \cap K \cap \operatorname{cl}(X)=\emptyset$.

By Lemma 3.3.6, we may assume; relabelling as necessary, that $(X,\{b\}, Y)$ is a vertical 3-separation such that $X \cup\{b\}$ is closed. Moreover, by 5.2.3.1, we maintain the property that $(X,\{b\}, Y)$ is balanced with $c \in Y$. By assumption, $\left\{k_{1}, k_{2}\right\} \not \subset S$ and $\left\{k_{1}, k_{2}\right\} \not \subset T$. Without loss of generality, $k_{1} \in X \cap S$ and $k_{2} \in X \cap T$. Also, $|Y \cap S \cap K|=|Y \cap T \cap K|=1$ because $(X,\{b\}, Y)$ and $(S,\{c\}, T)$ are balanced. So each of the intersections $X \cap S, X \cap T, Y \cap S$ and $Y \cap T$ are non-empty and each contains exactly one element of $K$.

5.2.3.2. $|Y \cap S|=1$ or $Y \cap S$ is a triad.

Proof. $\lambda(Y)=\lambda(S)=2$. Now $|E-(Y \cup S)|=|(X \cap T) \cup\{b\}| \geq 2$. So by uncrossing, $\lambda(Y \cap S) \leq 2$. Similarly, $\lambda((Y \cap S) \cup\{c\})=2$. Suppose $|Y \cap S| \geq 2$. Then $\lambda(Y \cap S)=\lambda((Y \cap S) \cup\{c\})=2$ because $M$ is 3 -connected. By Lemmas 3.2.7 and 2.5.4, $c \in \mathrm{cl}^{*}(Y \cap S)$. If $Y \cap S$ contains a circuit, then, as $|Y \cap S \cap K|=1$, applying Corollary 4.1.3, we deduce that $Y \cap S$ contains a triangle which is contained in a 4-point line. But $M$ has no such 4-point lines. So $Y \cap S$ is independent. As $c \in \operatorname{cl}^{*}(Y \cap S)-\operatorname{cl}(Y \cap S),(Y \cap S) \cup\{c\}$ is also independent. Recall that $(Y \cap S) \cup\{c\}$ is exactly 3-separating. As $M$ does not contain any 4-point colines, Lemma 3.2.6 implies that $|Y \cap S| \leq 2$. Therefore $|Y \cap S|=2$ and $(Y \cap S) \cup\{c\}$ is a triad. Otherwise, $|Y \cap S|=1$.

5.2.3.3. $|X \cap S| \geq 2$

Proof. If $|X \cap S|=1$, then, as $|S| \geq 3,(Y \cap S) \cup\{c\}$ must be a triad by 5.2.3.2. Now $(S,\{c\}, T)$ is a cyclic 3 -separation of a 3 -connected matroid with $|S|=3$, so $S$ must be a triangle. But then $S \cup\{c\}$ is a contradictory 4-element fan of $M$. Therefore $|X \cap S| \geq 2$. 
5.2.3.4. $\lambda(Y \cap T) \leq 2$ and $\lambda((Y \cap T) \cup\{c\})=\lambda((Y \cap T) \cup\{b, c\})=2$.

Proof. $\lambda(Y)=\lambda(T)=2$. By 5.2.3.3, $|E-(Y \cup T)|=|X \cap S| \geq 2$. Therefore, by uncrossing, $\lambda(Y \cap T) \leq 2$. Similarly, $|E-(Y \cup(T \cup\{c\}))|=\mid E-((Y \cup$ $\{b\}) \cup(T \cup\{c\}))|=| X \cap S \mid \geq 2$, so by uncrossing, and the fact that $M$ is 3-connected, $\lambda((Y \cap T) \cup\{c\})=\lambda((Y \cap T) \cup\{b, c\})=2$.

Suppose $|Y \cap T| \geq 2$. Then by 5.2.3.4, $\lambda(Y \cap T)=\lambda((Y \cap T) \cup\{c\})=$ $\lambda((Y \cap T) \cup\{b, c\})=2$. Applying Lemmas 3.2.7 and 2.5.4, we deduce that $b \in \operatorname{cl}(Y \cap T)$ and $c \in \operatorname{cl}^{*}(Y \cap T)$. Hence, if $|Y \cap T|=2,(Y \cap T) \cup\{b\}$ is a triangle. But then $((Y \cap T) \cup\{b\},\{c\}, X \cup S)$ is a cyclic 3-separation of $M$. As $M$ does not contain any 4-point lines and $|((Y \cap T) \cup\{b\}) \cap K|=1$, this contradicts Corollary 4.1.3. So $|Y \cap T| \neq 2$. If $|Y \cap T| \geq 3$ and $Y \cap T$ contains a circuit, then we again have a cyclic 3 -separation $(Y \cap T,\{c\}, X \cup S \cup\{b\})$ of $M$ that contradicts of Corollary 4.1.3. So $|Y \cap T| \geq 3 \Longrightarrow Y \cap T$ is independent. But then $(Y \cap T) \cup\{c\}$ is both independent and exactly 3separating, and Lemma 3.2.6 implies that $(Y \cap T) \cup\{c\}$ contains a 4-point coline; a contradiction.

The only remaining possibility is that $|Y \cap T|=1$. By 5.2.3.4, $\lambda((Y \cap$ $T) \cup\{c\})=\lambda((Y \cap T) \cup\{b, c\})=2$. Therefore, by Lemmas 3.2.7 and 2.5.4, $b \in \operatorname{cl}((Y \cap T) \cup\{c\})$. Hence $(Y \cap T) \cup\{b, c\}$ is a triangle. Now if $|Y \cap S|=1$, then $Y$ is a triad, but then $Y \cup\{b\}$ is a contradictory 4-element fan of $M$. So $(Y \cap S) \cup\{c\}$ must be a triad by 5.2.3.2. But then we find ourselves with a circuit $(Y \cap T) \cup\{b, c\}$ and a cocircuit $(Y \cap S) \cup\{c\}$ whose intersection is $\{c\}$. This contradicts Lemma 2.5.5. We conclude that either $\left\{k_{1}, k_{2}\right\} \subset S$ or $\left\{k_{1}, k_{2}\right\} \subset T$. As $c$ was an arbitrary member of $(E(M)-B)-K$, case (iii) is complete and the theorem holds.

\subsection{Path Width}

We now tie in the results of Sections 5.1 and 5.2 with the notion of path-width (see Definition 5.0.1), and conclude with a proof of Theorem 5.0.2.

Definition 5.3.1. A path of 3-separations in a matroid $M$ is an ordered partition $\mathbb{P}=\left(P_{0}, P_{1}, \ldots, P_{r}\right)$ of $E(M)$ with the property that for all $i \in$ $\{0, \ldots, r-1\}, \lambda\left(P_{0} \cup \ldots \cup P_{i}\right)=2$. The members of $\mathbb{P}$ are called steps of $\mathbb{P}$. Empty steps are permitted. 
Note that it follows from the above definition that if $\mathbb{P}=\left(P_{0}, \ldots, P_{r}\right)$ is a path of 3-separations in a matroid $M$, then $P_{0} \neq \emptyset \neq P_{r}$.

Note also that both vertical and cyclic 3 -separations are examples of paths of 3-separations.

Lemma 5.3.2. Let $\mathbb{P}=\left(P_{0}, \ldots, P_{r}\right)$ be a path of 3 -separations in a matroid $M$. If, for some $i \in\{1, \ldots, r-1\},\left|P_{i}\right|=1$ with $P_{i}=\left\{e_{i}\right\}$, then either

(i) $e_{i} \in \operatorname{cl}\left(P_{0} \cup \ldots \cup P_{i-1}\right) \cap \operatorname{cl}\left(P_{i+1} \cup \ldots \cup P_{r}\right)$, or

(ii) $e_{i} \in \operatorname{cl}^{*}\left(P_{0} \cup \ldots \cup P_{i-1}\right) \cap \operatorname{cl}^{*}\left(P_{i+1} \cup \ldots \cup P_{r}\right)$.

Proof. Suppose $P_{i}=\left\{e_{i}\right\}$ for some $i \in\{1, \ldots, r-1\}$. Then $\lambda\left(P_{0} \cup \ldots \cup P_{i-1}\right)=$ $\lambda\left(P_{0} \cup \ldots \cup P_{i-1} \cup\left\{e_{i}\right\}\right)=2$. Also, $\lambda\left(P_{i+1} \cup \ldots \cup P_{r}\right)=\lambda\left(P_{0} \cup \ldots \cup P_{i-1} \cup\left\{e_{i}\right\}\right)=2$, and $\lambda\left(P_{i+1} \cup \ldots \cup P_{r} \cup\left\{e_{i}\right\}\right)=\lambda\left(P_{0} \cup \ldots \cup P_{i-1}\right)=2$. It then follows from Lemma 3.2.7 that $e_{i} \in \mathrm{cl}^{(*)}\left(P_{0} \cup \ldots \cup P_{i-1}\right)$ and $e_{i} \in \mathrm{cl}^{(*)}\left(P_{i+1} \cup \ldots \cup P_{r}\right)$. If $e_{i} \in \operatorname{cl}\left(P_{0} \cup \ldots \cup P_{i-1}\right)$, then by Lemma 2.5.4, $e_{i} \in \operatorname{cl}\left(P_{i+1} \cup \ldots \cup P_{r}\right)$; which gives (i). Otherwise, $e_{i} \in \mathrm{cl}^{*}\left(P_{0} \cup \ldots \cup P_{i-1}\right)$, in which case, Lemma 2.5.4 implies that $e_{i} \in \mathrm{cl}^{*}\left(P_{i+1} \cup \ldots \cup P_{r}\right)$; giving (ii).

Lemma 5.3.3. A 3-connected matroid $M$ has path-width 3 if and only if there is a path $\mathbb{P}=\left(P_{0}, \ldots, P_{r}\right)$ of 3 -separations such that $\left|P_{0}\right|=\left|P_{r}\right|=2$, and, for all $i \in\{1, \ldots, r-1\},\left|P_{i}\right|=1$.

Proof. Let $M$ be a 3-connected matroid with path-width 3 . Let $\left(e_{1}, \ldots, e_{n}\right)$ be an ordering of $E(M)$ such that for all $i \in\{1, \ldots, n\}, \lambda\left(\left\{e_{1}, \ldots, e_{i}\right\}\right) \leq 2$. As $M$ is 3 -connected, it follows that for $i \in\{2, \ldots, n-2\}, \lambda\left(\left\{e_{1}, \ldots, e_{i}\right\}\right)=2$. Now letting $P_{0}=\left\{e_{1}, e_{2}\right\}, P_{n-3}=\left\{e_{n-1}, e_{n}\right\}$ and $P_{i}=\left\{e_{i+2}\right\}$ for $i \in\{1, \ldots, n-4\}$, we obtain a suitable path of 3 -separations $\left(P_{0}, P_{1}, \ldots, P_{n-4}, P_{n-3}\right)$.

Conversely, let $\mathbb{P}=\left(P_{0}, \ldots, P_{r}\right)$ be a path of 3-separations in a 3 -connected matroid $M$ such that $\left|P_{0}\right|=\left|P_{r}\right|=2$, and, for all $i \in\{1, \ldots, r-1\},\left|P_{i}\right|=1$. Letting $\left\{e_{1}, e_{2}\right\}=P_{0},\left\{e_{r+2}, e_{r+3}\right\}=P_{r}$ and $\left\{e_{i+2}\right\}=P_{i}$ for $i \in\{1, \ldots, r-1\}$, we obtain an ordering $\left(e_{1}, \ldots, e_{r+3}\right)$ of $E(M)$ which implies that $M$ has pathwidth 3 .

Lemma 5.3.4. Let $M$ be a 3-connected matroid such that $E(M)=L \cup \Gamma$ where $L$ is a 4-point line and $\Gamma$ is a 4-point coline. Then $M$ has path-width 3 . 
Proof. Letting $L=\left\{l_{1}, l_{2}, l_{3}, l_{4}\right\}$ and $\Gamma=\left\{\gamma_{1}, \gamma_{2}, \gamma_{3}, \gamma_{4}\right\}$, the ordering $\left(l_{1}, l_{2}, l_{3}, l_{4}, \gamma_{1}, \gamma_{2}, \gamma_{3}, \gamma_{4}\right)$ of $E(M)$ reveals that $M$ has path-width 3 .

Proposition 5.3.5. Let $\mathbb{P}=\left(P_{0}, \ldots, P_{r}\right)$ be a path of 3 -separations in a matroid $M$. Suppose $i \in\{1, \ldots, r-1\}, e \in P_{i}$, and that there exists a path of 3-separations $(X,\{e\}, Y)$ of $M$ with $P_{0} \subseteq X, P_{r} \subseteq Y$ and $e \in \operatorname{cl}(X) \cap \operatorname{cl}(Y)$. Then there exists a path of 3-separations $\left(X^{\prime},\{e\}, Y^{\prime}\right)$ with $P_{0} \cup \ldots \cup P_{i-1} \subseteq X^{\prime}$, $P_{i+1} \cup \ldots \cup P_{r} \subseteq Y^{\prime}$, and $e \in \operatorname{cl}\left(X^{\prime}\right) \cap \operatorname{cl}\left(Y^{\prime}\right)$.

Proof. Let $S=P_{0} \cup \ldots \cup P_{i-1}$ and $T=P_{i+1} \cup \ldots \cup P_{r}$.

5.3.5.1. There exists a path of 3-separations $\left(X_{0},\{e\}, Y_{0}\right)$ of $M$ such that $S \subseteq X_{0}, P_{r} \subseteq Y_{0}$, and $e \in \operatorname{cl}\left(X_{0}\right) \cap \operatorname{cl}\left(Y_{0}\right)$.

Proof. We have $\lambda(Y \cup\{e\})=\lambda\left(P_{i} \cup T\right)=2$. Note that $P_{0} \subseteq X \cap S$, and $P_{0}$ contains at least two elements. Now

$$
\left|E(M)-\left((Y \cup\{e\}) \cup\left(P_{i} \cup T\right)\right)\right|=|X \cap S| \geq\left|P_{0}\right| \geq 2
$$

and it follows by uncrossing that $\lambda\left((Y \cup\{e\}) \cap\left(P_{i} \cup T\right)\right) \leq 2$. So $(X \cup S,(Y \cup$ $\left.\{e\}) \cap\left(P_{i} \cup T\right)\right)$ is a 3 -separating partition of $M$. We know that $\left|P_{0}\right| \geq 2$ and $\left|P_{r}\right| \geq 2$. Also, $P_{0} \subseteq X \cup S$ and $P_{r} \subseteq(Y \cup\{e\}) \cap\left(P_{i} \cup T\right)$. Therefore $\left(X \cup S,(Y \cup\{e\}) \cap\left(P_{i} \cup T\right)\right)$ is a 3-separation of $M$. But $e \in \operatorname{cl}(X) \subseteq \operatorname{cl}(X \cup S)$, and it follows from Lemmas 2.5.4 and 3.2.8 that $e \in \operatorname{cl}\left(\left((Y \cup\{e\}) \cap\left(P_{i} \cup\right.\right.\right.$ $T))-\{e\})$ and that $\left(X \cup S,\{e\}, Y \cap\left(P_{i} \cup T\right)\right)$ is a path of 3-separations. Letting $X_{0}=X \cup S$ and $Y_{0}=Y \cap\left(P_{i} \cup T\right)$, the result follows.

Now $\lambda\left(X_{0} \cup\{e\}\right)=\lambda\left(P_{i} \cup S\right)=2$, and $\left|E(M)-\left(\left(X_{0} \cup\{e\}\right) \cup\left(P_{i} \cup S\right)\right)\right|=$ $\left|Y_{0} \cap T\right| \geq\left|P_{r}\right| \geq 2$, so that, by uncrossing, $\lambda\left(\left(X_{0} \cup\{e\}\right) \cap\left(P_{i} \cup S\right)\right) \leq 2$. As $P_{0} \subseteq\left(X_{0} \cup\{e\}\right) \cap\left(P_{i} \cup S\right)$, it must be that $\lambda\left(\left(X_{0} \cup\{e\}\right) \cap\left(P_{i} \cup S\right)\right)=2$. Therefore $\left(\left(X_{0} \cup\{e\}\right) \cap\left(P_{i} \cup S\right), Y_{0} \cup T\right)$ is an exact 3-separation of $M$. As $e \in \operatorname{cl}\left(Y_{0} \cup T\right)$, it then follows from Lemmas 2.5.4 and 3.2.8 that $e \in$ $\operatorname{cl}\left(\left(\left(X_{0} \cup\{e\}\right) \cap\left(P_{i} \cup S\right)\right)-\{e\}\right)$ and that $\left(X_{0} \cap\left(P_{i} \cup S\right),\{e\}, Y_{0} \cup T\right)$ is a path of 3-separations. Observing that $S \subseteq X_{0} \cap\left(P_{i} \cup S\right)$ and $T \subseteq Y_{0} \cup T$, we conclude that the theorem holds.

Corollary 5.3.6. Let $\mathbb{P}=\left(P_{0}, \ldots, P_{r}\right)$ be a path of 3-separations in a matroid M. Suppose $i \in\{1, \ldots, r-1\}, e \in P_{i}$, and that there exists a path of 3separations $(X,\{e\}, Y)$ in $M$ with $P_{0} \subseteq X, P_{r} \subseteq Y$ and $e \in \mathrm{cl}^{*}(X) \cap \operatorname{cl}^{*}(Y)$. Then there exists a path of 3-separations $\left(X^{\prime},\{e\}, Y^{\prime}\right)$ with $P_{0} \cup \ldots \cup P_{i-1} \subseteq X^{\prime}$, $P_{i+1} \cup \ldots \cup P_{r} \subseteq Y^{\prime}$, and $e \in \mathrm{cl}^{*}\left(X^{\prime}\right) \cap \mathrm{cl}^{*}\left(Y^{\prime}\right)$. 
Proof. This follows by duality from Proposition 5.3.5.

Corollary 5.3.7. Let $\mathbb{P}=\left(P_{0}, \ldots, P_{r}\right)$ be a path of 3-separations in a matroid M. Suppose $i \in\{1, \ldots, r-1\}, e \in P_{i}$, and that there exists a path of 3 separations $(X,\{e\}, Y)$ in $M$ with $P_{0} \subseteq X$ and $P_{r} \subseteq Y$. Then there exists a path of 3-separations $\left(X^{\prime},\{e\}, Y^{\prime}\right)$ with $P_{0} \cup \ldots \cup P_{i-1} \subseteq X^{\prime}$ and $P_{i+1} \cup \ldots \cup P_{r} \subseteq$ $Y^{\prime}$.

Proof. This is a consequence of Lemma 5.3.2, together with the results of Proposition 5.3.5 and Corollary 5.3.6.

Corollary 5.3.8. Let $\mathbb{P}=\left(P_{0}, \ldots, P_{r}\right)$ be a path of 3-separations in a matroid M. Suppose $i \in\{1, \ldots, r-1\}, e \in P_{i}$, and that there exists a path of 3 separations $(X,\{e\}, Y)$ of $M$ with $P_{0} \subseteq X$ and $P_{r} \subseteq Y$. Then $\mathbb{P}$ refines to a path $\left(P_{0}, \ldots, P_{i-1}, P_{i}^{\prime},\{e\}, P_{i}^{\prime \prime}, P_{i+1}, \ldots, P_{r}\right)$ of 3-separations where $P_{i}^{\prime} \cup\{e\} \cup$ $P_{i}^{\prime \prime}=P_{i}$.

Proof. By Corollary 5.3.7, we have a path of 3-separations $\left(X^{\prime},\{e\}, Y^{\prime}\right)$ of $M$ with $\left(P_{0} \cup \ldots \cup P_{i-1}\right) \subseteq X^{\prime}$ and $\left(P_{i+1} \cup \ldots \cup P_{r}\right) \subseteq Y^{\prime}$. Letting $P_{i}^{\prime}=X^{\prime}-\left(P_{0} \cup \ldots \cup P_{i-1}\right)$ and $P_{i}^{\prime \prime}=Y^{\prime}-\left(P_{i+1} \cup \ldots \cup P_{r}\right)$, it follows that $\left(P_{0}, \ldots, P_{i-1}, P_{i}^{\prime},\{e\}, P_{i}^{\prime \prime}, P_{i+1}, \ldots, P_{r}\right)$ is a path of 3 -separations. Moreover, $P_{i}^{\prime} \cup\{e\} \cup P_{i}^{\prime \prime}=P_{i}$.

Corollary 5.3.9. Let $M$ be a 3-connected matroid with disjoint sets $\left\{a_{1}, a_{2}\right\} \subset E(M)$ and $\left\{z_{1}, z_{2}\right\} \subset E(M)$. Suppose that for every $e \in$ $E(M)-\left\{a_{1}, a_{2}, z_{1}, z_{2}\right\}$, there exists a path of 3 -separations $(X,\{e\}, Y)$ of $M$ such that $\left\{a_{1}, a_{2}\right\} \subseteq X$ and $\left\{z_{1}, z_{2}\right\} \subseteq Y$. Then $M$ has path-width 3 .

Proof. As $M$ is 3-connected, $\left(\left\{a_{1}, a_{2}\right\}, E(M)-\left\{a_{1}, a_{2}, z_{1}, z_{2}\right\},\left\{z_{1}, z_{2}\right\}\right)$ is a path of 3-separations. If $E(M)=\left\{a_{1}, a_{2}, z_{1}, z_{2}\right\}$, the result is immediate. So suppose that $e \in E(M)-\left\{a_{1}, a_{2}, z_{1}, z_{2}\right\}$. Applying Corollary 5.3.8, we obtain a refinement of our original path of 3 -separations. Now successively applying Corollary 5.3.8 to each of the other elements of $E(M)-\left\{a_{1}, a_{2}, z_{1}, z_{2}\right\}$; each time with respect to our new refined path of 3 -separations, we eventually obtain a path of 3 -separations $\mathbb{P}=\left(\left\{a_{1}, a_{2}\right\}, P_{1}, \ldots, P_{q},\left\{z_{1}, z_{2}\right\}\right)$ where, for all $i \in\{1, \ldots, q\}$, the step $P_{i}$ is either a singleton, or empty. If we then remove all empty steps from $\mathbb{P}$, we obtain a path of 3 -separations $\mathbb{P}^{\prime}=$ $\left(\left\{a_{1}, a_{2}\right\}, P_{1}^{\prime}, \ldots, P_{n}^{\prime},\left\{z_{1}, z_{2}\right\}\right)$ in which $P_{i}$ is a singleton for all $i \in\{1, \ldots, n\}$. By Lemma 5.3.3, $M$ has path-width 3 . 
Proof of Theorem 5.0.2. If $M$ is the union of a 4-point line and a 4-point coline, then the result follows immediately from Lemma 5.3.4. So assume that this is not the case. Let $K=\left\{k_{1}, k_{2}, k_{3}, k_{4}\right\}$. Recall that vertical 3-separations and cyclic 3-separations are both examples of paths of 3-separations. Therefore it follows from Theorem 5.2.3 that for each $e_{i} \in E(M)-K$, there exists a path of 3 -separations $\left(X_{i},\left\{e_{i}\right\}, Y_{i}\right)$ of $M$ such that $\left\{k_{1}, k_{2}\right\} \subset X_{i}$ and $\left\{k_{3}, k_{4}\right\} \subset Y_{i}$. Thus, by Corollary 5.3.9, $M$ has path-width 3 . 


\section{Chapter 6}

\section{Future Work}

\subsection{Some Conjectures}

Our motivation for wanting to maintain 3-connectivity upon removing elements from matroids arose primarily from our desire to obtain matroids that are uniquely representable. But as discussed in Section 2.7, in general, for a matroid to have any sense of unique representability over $G F(q)$, not only must that matroid be 3 -connected, but we also require that it contain a stabilizer for $G F(q)$ as a minor. While we have shown that for a suitable matroid $M$ with arbitrary basis $B$, there always exists at least four elements which are removable with respect to $B$; thereby maintaining 3 -connectivity, in principle, it is possible that removing any one of these elements means losing the existence of a stabilizer as a minor; hence losing our sense of unique representability. However, we believe that something can be said provided there exists some element of $E(M)$ that can be deleted or contracted as appropriate while maintaining the stabilizer as a minor. We make the following conjecture, which we believe should follow from results established in this thesis.

Conjecture 6.1.1. Let $M$ be a 3-connected matroid with no 4-element fans, and let $B$ be a basis of $M$. Let $N$ be a 3-connected minor of $M$. If there exists some $b \in B$ such that $M / b$ has an $N$-minor, or there exists some $c \in E(M)-B$ such that $M \backslash c$ has an $N$-minor, then there exists distinct $k_{1}, k_{2} \in E(M)$ such that for $i \in\{1,2\}$, 
(i) $\operatorname{si}\left(M / k_{i}\right)$ is 3-connected with an $N$-minor if $k_{i} \in B$, and

(ii) $\operatorname{co}\left(M \backslash k_{i}\right)$ is 3-connected with an $N$-minor if $k_{i} \in E(M)-B$.

We showed in Chapter 5 that for a suitable matroid $M$ and basis $B$, if there are exactly four elements which are removable with respect to $B$, then $M$ necessarily has path-width 3 . Now the class of matroids with pathwidth 3 is well understood (see [1],[6]). Therefore, one should be able to give a stronger, more explicit, description of the class of matroids which have minimal removability with respect to some basis. For the moment, we leave this as an open problem.

Also, assuming that Conjecture 6.1.1 holds, it would be of interest to study the structure that is imposed upon $M$ if there are exactly two elements which we can remove with respect to $B$ while maintaining an $N$-minor. Furthermore, it is plausible that from a confirmation of Conjecture 6.1.1, we may be able to strengthen the result by removing the restriction that we need to simplify and cosimplify to remain 3-connected. Indeed, Oxley et al [10] showed the following:

Theorem 6.1.2. Let $M$ be a 3-connected matroid with no 4-element fans, and let $N$ be a 3-connected minor of $M$. Let $B$ be a basis of $M$, and assume that either there is an element $b_{1}$ of $B$ such that $M / b_{1}$ has an $N$-minor, or there is an element $b_{1}^{*}$ of $E(M)-B$ such that $M \backslash b_{1}^{*}$ has an $N$-minor. Then either:

(i) B contains an element $b$ such that $M / b$ is 3-connected with an $N$-minor, or

(ii) $E(M)-B$ contains an element $b^{*}$ such that $M \backslash b^{*}$ is 3-connected with an $N$-minor.

We next propose such a strengthening of Conjecture 6.1 .1 ; analogous to Theorem 6.1.2. However it seems quite possible that in this case, extra constraints on the matroid $M$ may be required.

Conjecture 6.1.3. Let $M$ be a 3-connected matroid with no 4-element fans, and let $B$ be a basis of $M$. Let $N$ be a 3-connected minor of $M$. If there exists some $b \in B$ such that $M / b$ has an $N$-minor, or there exists some $c \in E(M)-B$ such that $M \backslash c$ has an $N$-minor, then there exists distinct $k_{1}, k_{2} \in E(M)$ such that for $i \in\{1,2\}$, 
(i) $M / k_{i}$ is 3-connected with an $N$-minor if $k_{i} \in B$, and

(ii) $M \backslash k_{i}$ is 3-connected with an $N$-minor if $k_{i} \in E(M)-B$.

In the event that Conjecture 6.1.3 was shown to hold, we could then take $N$ to be the empty matroid in 6.1.3 and obtain the following as a corollary.

Conjecture 6.1.4. Let $M$ be a 3-connected matroid with no 4-element fans, and let $B$ be a basis of $M$. Then there exists distinct $k_{1}, k_{2} \in E(M)$ such that for $i \in\{1,2\}$,

(i) $M / k_{i}$ is 3-connected if $k_{i} \in B$, and

(ii) $M \backslash k_{i}$ is 3-connected if $k_{i} \in E(M)-B$.

Finally, we note an observation on Theorem 5.0.2. We showed that if a 3-connected matroid $M$ with no 4-element fans, and some given basis $B$ contains exactly four elements which are removable with respect to $B$, then the matroid $M$ itself has path-width 3 . But the notion of removability is relative to the choice of basis one makes. It is certainly possible that upon switching to another basis $B^{\prime}$, we find that $M$ contains more than four removable elements. Potentially, one could examine the entire collection of bases of suitable matroids. Perhaps one could deduce that, for any 3-connected matroid $M$ with no 4-element fans, there exists some basis $B^{\exists}$ such that $M$ contains more than four elements which are removable with respect to $B^{\exists}$.

Question 6.1.5. Does there exist a 3-connected matroid $M$ with no 4element fans such that for every basis $B$ of $M$, there exists exactly four elements of $E(M)$ which are removable with respect to $B$ ? If so, what additional structure must such an $M$ have? 


\section{Bibliography}

[1] Beavers, B., Oxley, J. Constructive characterizations of 3-connected matroids of path width three. Europ. J. Combin. 29 (2008) 1643-1661.

[2] Bixby, R. E., On Reid's characterization of the ternary matroids. J. Combin. Theory Ser. B 26 (1979) 174-204.

[3] Bixby, R. E., A simple theorem on 3-connectivity. Linear Algebra Appl. 45 (1982) 123-126.

[4] Geelen, J. F., Gerards, A. M. H., and Kapoor, A., The excluded minors for GF(4)-representable matroids. J. Combin. Theory Ser. B 79 (2000) 247-299.

[5] Geelen, J. F., Whittle, G., Inequivalent representations of matroids over prime fields. (in preparation)

[6] Hall, R., Oxley, J., and Semple, C., The structure of 3-connected matroids of path width three. Europ. J. Combin. 28 (2007) 964-989.

[7] Lazarson, T., The representation problem for independence functions. J. London Math. Soc. 33 (1958), 21-25.

[8] Mayhew, D., Matroid theory (unpublished lecture notes)

[9] Oxley, J. G., Matroid Theory. Oxford University Press, New York, 1992.

[10] Oxley, J., Semple, C., Whittle, G., Maintaining 3-connectivity relative to a fixed basis. Adv. Appl. Math. 41 (2008) 1-9.

[11] Oxley, J. G., Vertigan, D. L., and Whittle, G. P., On inequivalent representations of matroids over finite fields. J. Combin. Theory Ser. B 67 (1996) 325-343. 
[12] Rota, G. -C. Combinatorial theory, old and new. In Actes du Congrés International des Mathématiciens (Nice 1970), Tom 3, pp. 229-233. Gauthier-Villars, Paris (1971).

[13] Seymour, P. D., Matroid representation over GF(3). J. Combin. Theory Ser. B 26 (1979), 159-173.

[14] Tutte, W. T., A homotopy theorem for matroids, I, II. Trans. Amer. Math. Soc. 88 (1958) 144-174.

[15] Vámos, P., On the representation of independence structures (unpublished manuscript).

[16] Whitney, H., On the abstract properties of linear dependence. Amer. J. Math. 57 (1935), 509-533.

[17] Whittle, G. P., Stabilizers of classes of representable matroids. J. Combin. Theory Ser. B 77 (1999), 39-72. 